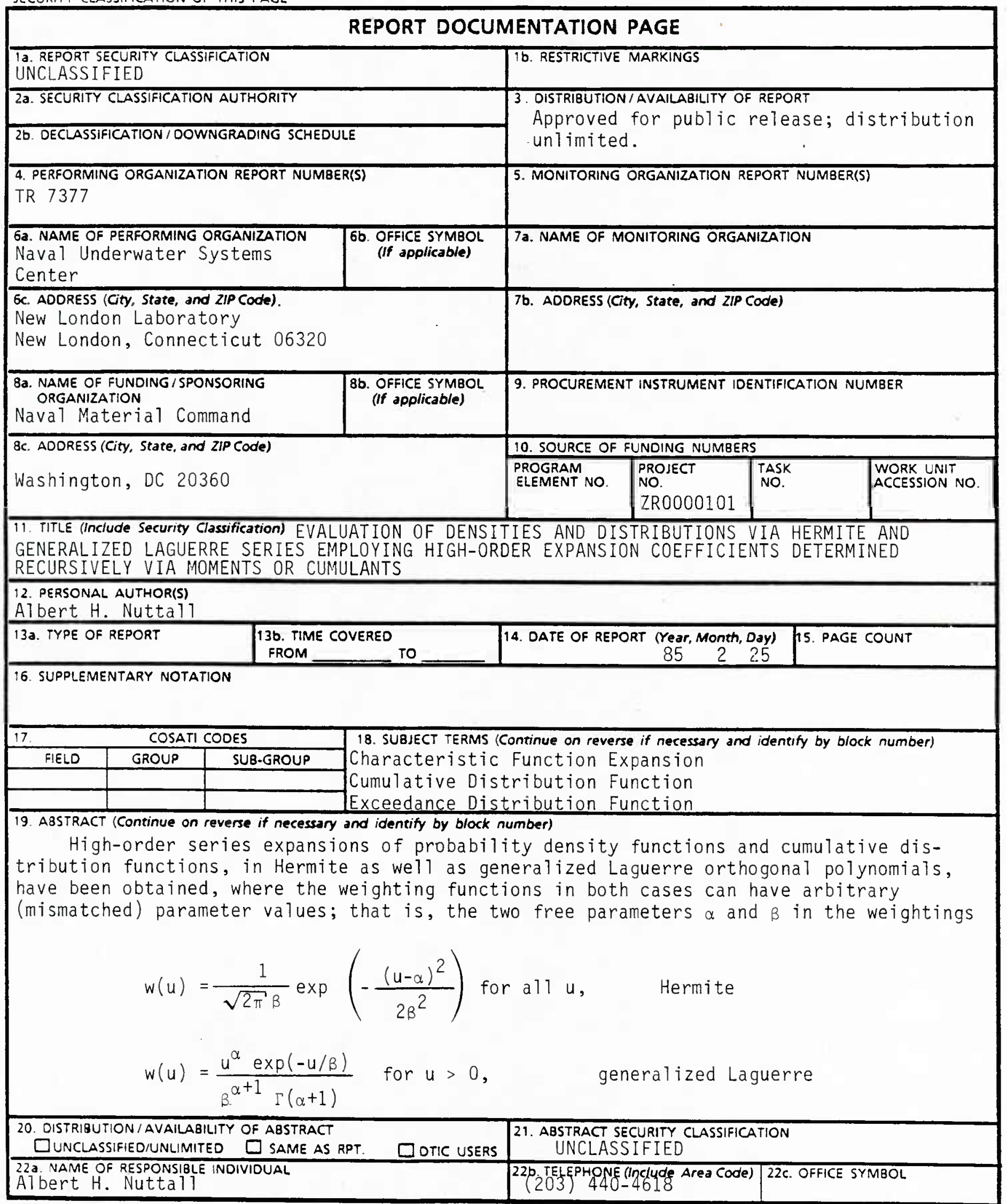

DD FORM 1473, 84 MAR

83 APR edition may be used until exhausted. All other editions are obsolete.
SECURITY CLASSIFICATION OF THIS PAGE

UNCLASS I FIED 
18. (Cont'd)

False Alarm/Detection Probability

Generalized Laguerre Expansions

Hermite Expansions

High-Order Moments/Cumulants

Mismatched Parameter Values
Probability Density Function

Recursive Expansion Coefficients

Recursive Moments/Cumulants

Rician Variate

Shot Noise

19. (Cont'd)

need not be chosen so that the first two expansion coefficients $b_{1}$ and ' $b_{2}$ in the orthonormal series are zero. (The zero-th order expansion coefficient bo is never zero.) Nonetheless all the available $\mathrm{N}$ Towest-order moments of the approximating probability density function are maintained identical to those of the given probability density function, regardless of the weighting employed and any of its free parameter values.

It has been discovered that deliberate mismatch of $\alpha$ and $\beta$ results in faster-decaying coefficient sequences $\left\{b_{n}\right\}{ }_{0}^{N}$ than when $\alpha$ and $\beta$ are chosen to make $b_{1}=b_{2}=0$, which is a common choice. For example, the central limit theorem is just such a case, where $\alpha$ and $\beta$ in the Hermite expansion are taken as the mean and standard deviation, respectively, and the number of moments employed is limited to just order $\mathrm{N}=2$.

A fast trial-and-error procedure is used in general to determine good values of weighting parameters $\alpha$ and $B$. The only statistics needed about the given probability density function or cumulative distribution function are either its moments or cumulants, through order $N$. Furthermore, all the results presented actually apply to functions which have arbitrary area (not necessarily equal to unity) and to functions which can become negative. In fact, one of the applications considered is to a shot noise process where the continuous part of the probability density function has area less than 1 , and which is well approximated by a generalized Laguerre series expansion.

The high-order expansion coefficients for both the Hermite and generalized Laguerre series can each be obtained by any one of three fast recursive procedures (all of which have been programmed, and for which program listings are presented):

(a) recursively via cumulants,

(b) directly via moments,

(c) recursively via moments.

The forms of these three recursive procedures differ in the Hermite vs. Laguerre cases; however, they are basically either convolutions or finite alternating series with binomial coefficients. The occurrence and quantitative value of round-off error for large $N$ is easily discerned in a plot of the expansion coefficient sequence for each choice of $\alpha$ and $\beta$, and for each of the three procedures, as well as for both types of series expansions.

Comparisons of the accuracy of the three alternative recursive procedures reveals that expansion coefficients determined recursively via cumulants are generally most accurate and least susceptible to round-off error. Numerous examples of series expansions of probability density functions and cumulative distribution functions are given, including one with $N=150$ terms, where the last expansion coefficient is of size $1 E-10$ relative to the leading coefficient $b_{0}$. Estimates of the error associated with the approximations obtained by the Hermite and generalized Laguerre series are derived and compared with results of several examples. 
LIERARY

RESEARCH REPORTS DIVISION

NWVAL POSTGRADUATE SCHOOL

MONIEREY, CALIFORNIA 939.93

\section{Evaluation of Densities and Distributions via Hermite and Generalized Laguerre Series Employing High-Order Expansion Coefficients Determined Recursively via Moments or Cumulants}

Albert H. Nuttall

Surface Ship Sonar Department

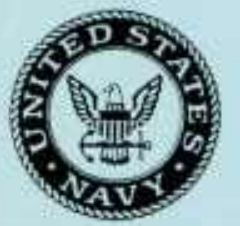

,Naval Underwater Systems Center, Newport, Rhode Island/New London, Connecticut 
PREFACE

This research was conducted under NUSC Project No. A75205, Subproject No. ZR0000101, "Applications of Statistical Communication Theory to Acoustic Signal Processing," Principal Investigator or. Albert H. Nuttall (Code 33), and under NUSC Project No. A65090, Subproject No. ZRO00-01, "Evaluation of Incoherent Field in the Arctic Environment," Principal Investigator R. L. Deavenport (Code 3332), Program Manager Gary Morton, Naval Material Command (MAT 05).

The Technical Reviewer for this report was Dr. Roy L. Streit (Code 3232).

Reviewed and Approved: 28 February 1985

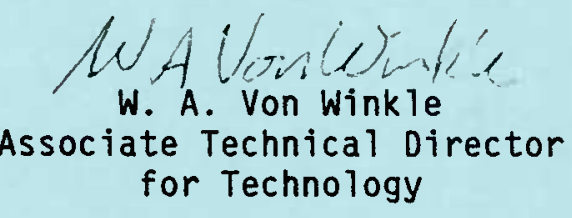

The author of this report is located at the New London Laboratory, Naval Underwater Systems Center. New London, Connecticut 06320. 
LIST OF ILLUSTRATIONS $\ldots \ldots \ldots \ldots \ldots \ldots \ldots \ldots \ldots \ldots \ldots \ldots \ldots \ldots \ldots \ldots \ldots \ldots \ldots \ldots$

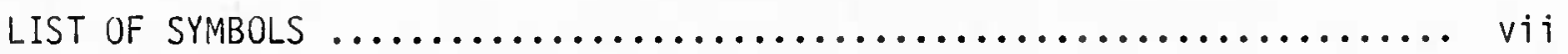

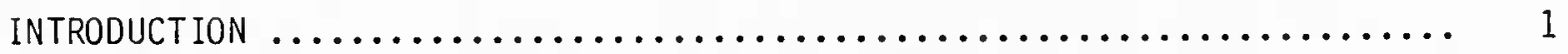

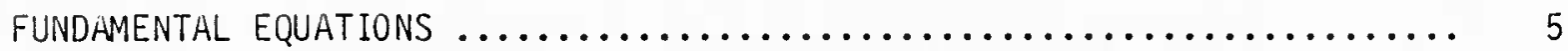

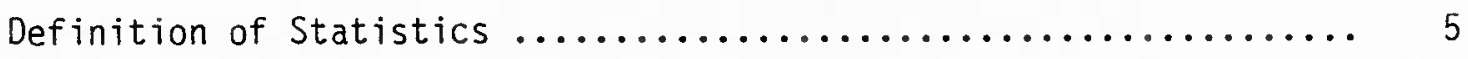

Weighting Function Properties ......................... 7

Approximation Procedure $\ldots \ldots \ldots \ldots \ldots \ldots \ldots \ldots \ldots \ldots \ldots \ldots \ldots$

Equality of Probability Density Function Moments ............ 10

Parameters of Given Probability Density Function $p$............ 11

General Results for Three Lowest-Order Polynomials $Q_{n} \ldots \ldots \ldots \ldots$

Special Choices of Weighting Parameters $\ldots \ldots \ldots \ldots \ldots \ldots \ldots \ldots$

Example of Divergent Error Integral for $b_{1}=0, b_{2}=0 \ldots \ldots \ldots \ldots .15$

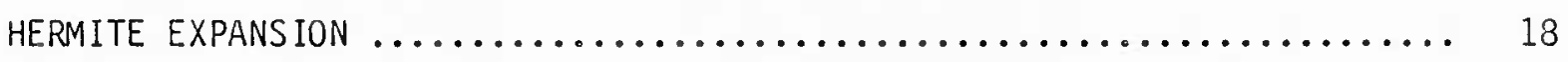

Properties of Polynomials and Expansions $\ldots \ldots \ldots \ldots \ldots \ldots \ldots \ldots$

Expansion of Characteristic Function $f \ldots \ldots \ldots \ldots \ldots \ldots \ldots \ldots . \ldots \ldots$

Coefficients Recursively via Cumulants $\ldots \ldots \ldots \ldots \ldots \ldots \ldots \ldots \ldots$

Coefficients Directly via Moments $\ldots \ldots \ldots \ldots \ldots \ldots \ldots \ldots \ldots \ldots \ldots$

Coefficients Recursively via Moments $\ldots \ldots \ldots \ldots \ldots \ldots \ldots \ldots \ldots$

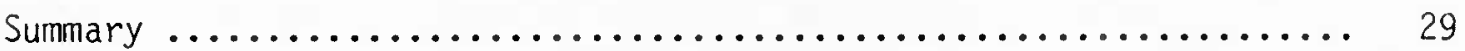

GENERALIZED LAGUERRE EXPANSION $\ldots \ldots \ldots \ldots \ldots \ldots \ldots \ldots \ldots \ldots \ldots \ldots \ldots \ldots \ldots \ldots \ldots \ldots \ldots$

Properties of Polynomials and Expansions ................. 31

Expansion of Characteristic Function $f \ldots \ldots \ldots \ldots \ldots \ldots \ldots \ldots \ldots \ldots \ldots$ 
TR 7377

\section{TABLE OF CONTENTS (Cont'd)}

PAGE

Coefficients Recursiveiy via Cumulants $\ldots \ldots \ldots \ldots \ldots \ldots \ldots \ldots . . . . .637$

Coefficients Directly via Moments $\ldots \ldots \ldots \ldots \ldots \ldots \ldots \ldots \ldots . . . \ldots$

Coefficients Recursively via Moments $\ldots \ldots \ldots \ldots \ldots \ldots \ldots \ldots \ldots . . .62$

Summary $\ldots \ldots \ldots \ldots \ldots \ldots \ldots \ldots \ldots \ldots \ldots \ldots \ldots \ldots \ldots \ldots \ldots, 43$

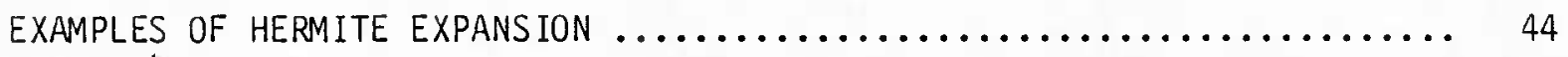

Examp te $A$.......................................... 44

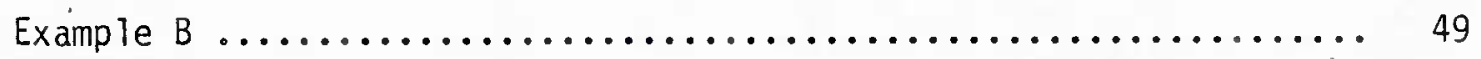

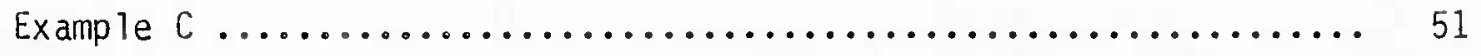

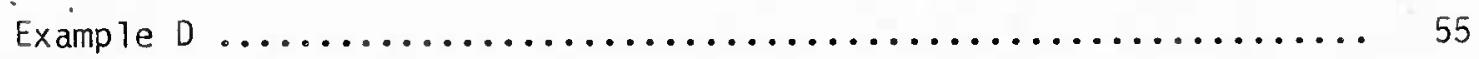

EXAMPLES OF GENERALIZED LAGUERRE EXPANSION $\ldots \ldots \ldots \ldots \ldots \ldots \ldots \ldots \ldots .61$

Example $E$....................................... 61

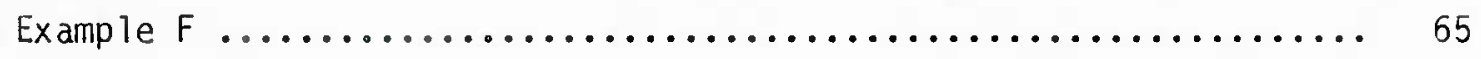

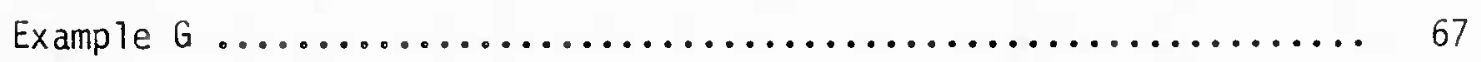

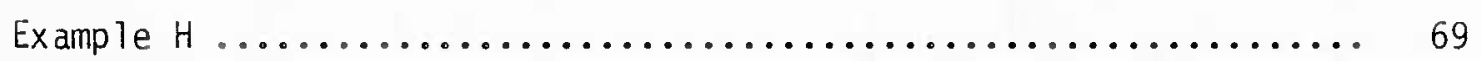

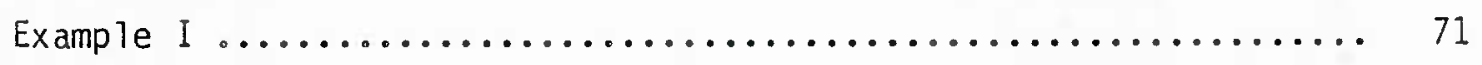

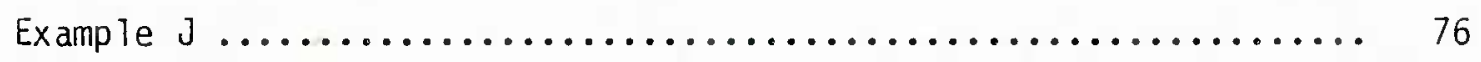

ESTIMATED ERRORS OF APPROXIMATIONS $\ldots \ldots \ldots \ldots \ldots \ldots \ldots \ldots \ldots \ldots \ldots \ldots, 80$

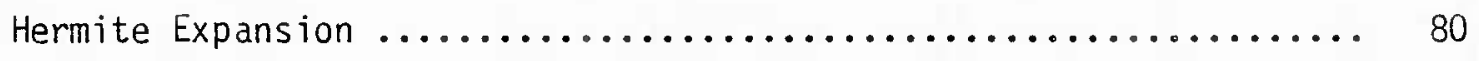

Generalized Laguerre Expansion $\ldots \ldots \ldots \ldots \ldots \ldots \ldots \ldots \ldots \ldots \ldots . .64$

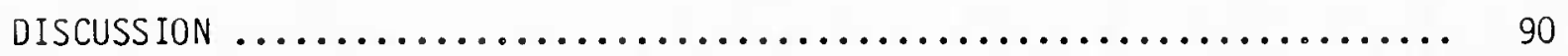


TABLE OF CONTENTS (Cont'd)

PAGE

\section{APPENDICES}

A. COEFFICIENT RECURSION FOR EXPONENTIAL OF POWER SERIES $\ldots \ldots \ldots \ldots . . .93$

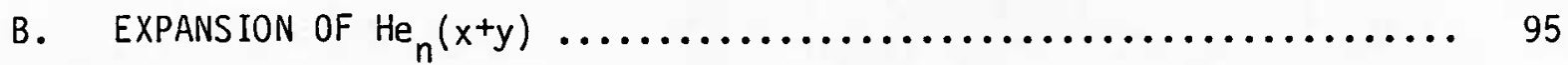

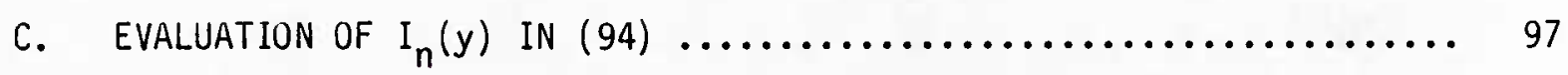

D. FOURIER TRANSFORM OF GENERAL IZED LAGUERRE POLYNOMIAL $\ldots \ldots \ldots \ldots . . . .99$

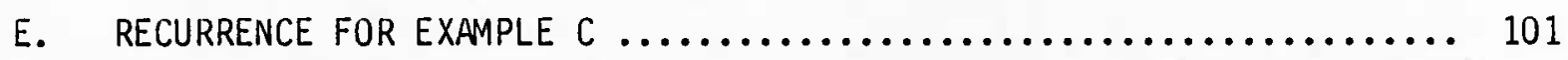

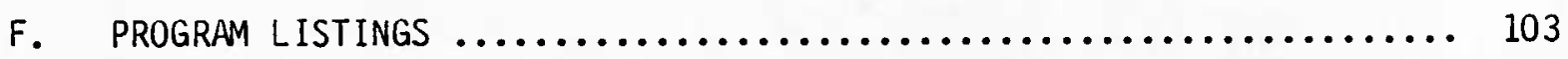

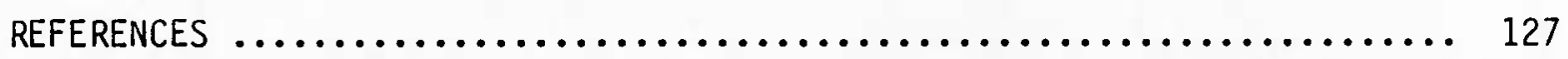

iii/iv 
1. Hermite Coefficients for Example $A \ldots \ldots \ldots \ldots \ldots \ldots \ldots \ldots \ldots \ldots$

2. Distributions for Example $A \ldots \ldots \ldots \ldots \ldots \ldots \ldots \ldots \ldots \ldots \ldots \ldots \ldots$

3. Hermite Coefficients for Example B .................... 50

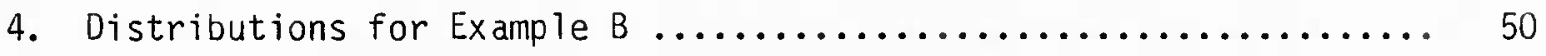

5. Hermite Coefficients for Example $C \ldots \ldots \ldots \ldots \ldots \ldots \ldots \ldots \ldots \ldots$

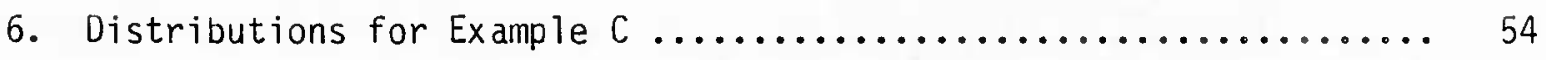

7. Hermite Coefficients for Example $0 \ldots \ldots \ldots \ldots \ldots \ldots \ldots \ldots \ldots \ldots$

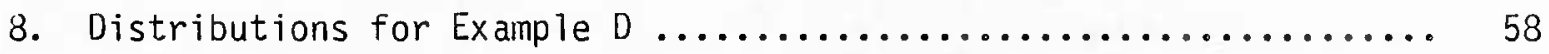

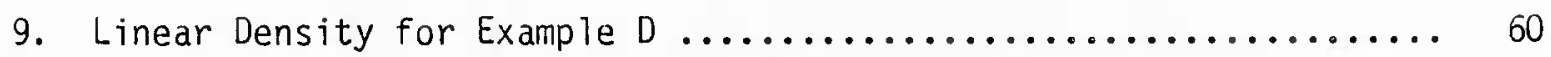

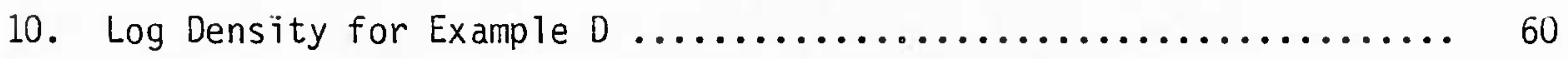

11. Generalized Laguerre; Example $E \ldots \ldots \ldots \ldots \ldots \ldots \ldots \ldots \ldots \ldots \ldots$

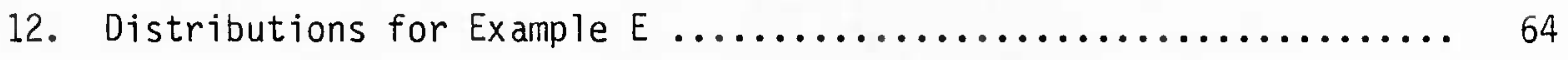

13. Generalized Laguerre; Example $F \ldots \ldots \ldots \ldots \ldots \ldots \ldots \ldots \ldots \ldots$

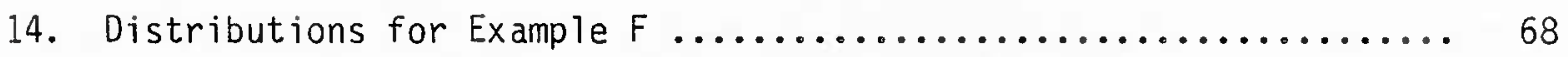

15. Generalized Laguerre; Example $6 \ldots \ldots \ldots \ldots \ldots \ldots \ldots \ldots \ldots \ldots$

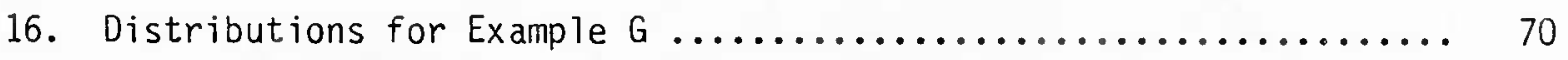

17. Generalized Laguerre; Example H ..................... 72

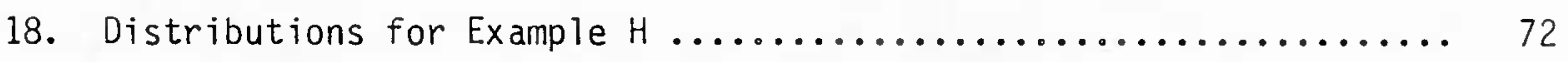

19. Generalized Laguerre; Example I $\ldots \ldots \ldots \ldots \ldots \ldots \ldots \ldots \ldots \ldots$

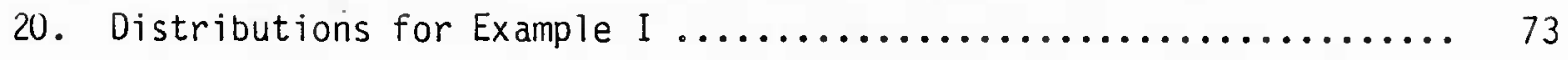

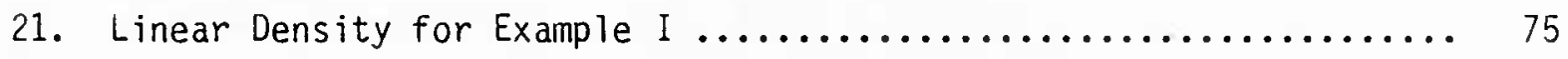

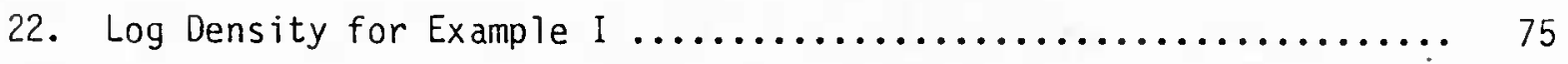


TR 7377

$$
\text { LIST OF ILLUSTRATIONS (Cont'd) }
$$

FIGURE

23. Generalized Laguerre; Example J ..................... 77

24. Distributions for Example $J \ldots \ldots \ldots \ldots \ldots \ldots \ldots \ldots \ldots \ldots \ldots \ldots \ldots$

25. Linear Density for Example J ........................ 79

26. Log Density for Example J ............................. 79

27. Estinated Error of Figure $8 \ldots \ldots \ldots \ldots \ldots \ldots \ldots \ldots \ldots \ldots \ldots \ldots \ldots \ldots \ldots \ldots$

28. Estimated Error of Figure $10 \ldots \ldots \ldots \ldots \ldots \ldots \ldots \ldots \ldots \ldots \ldots \ldots \ldots \ldots \ldots \ldots \ldots$

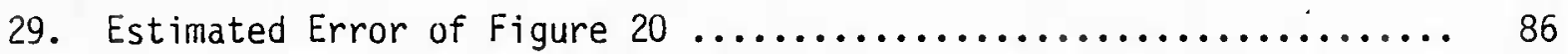

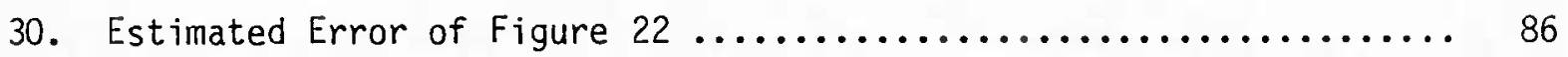

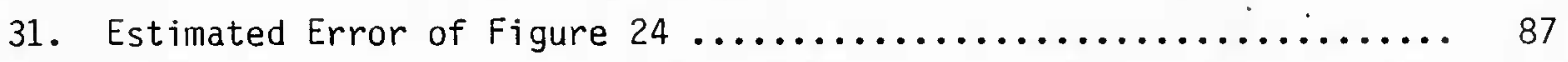

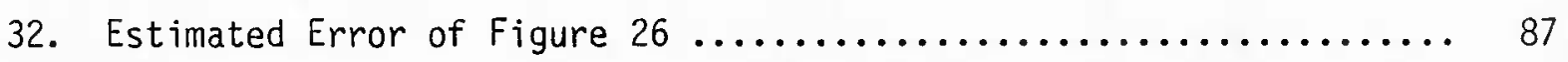

33. Estimated Error of Figure $16 \ldots \ldots \ldots \ldots \ldots \ldots \ldots \ldots \ldots \ldots \ldots \ldots \ldots \ldots \ldots \ldots$ 


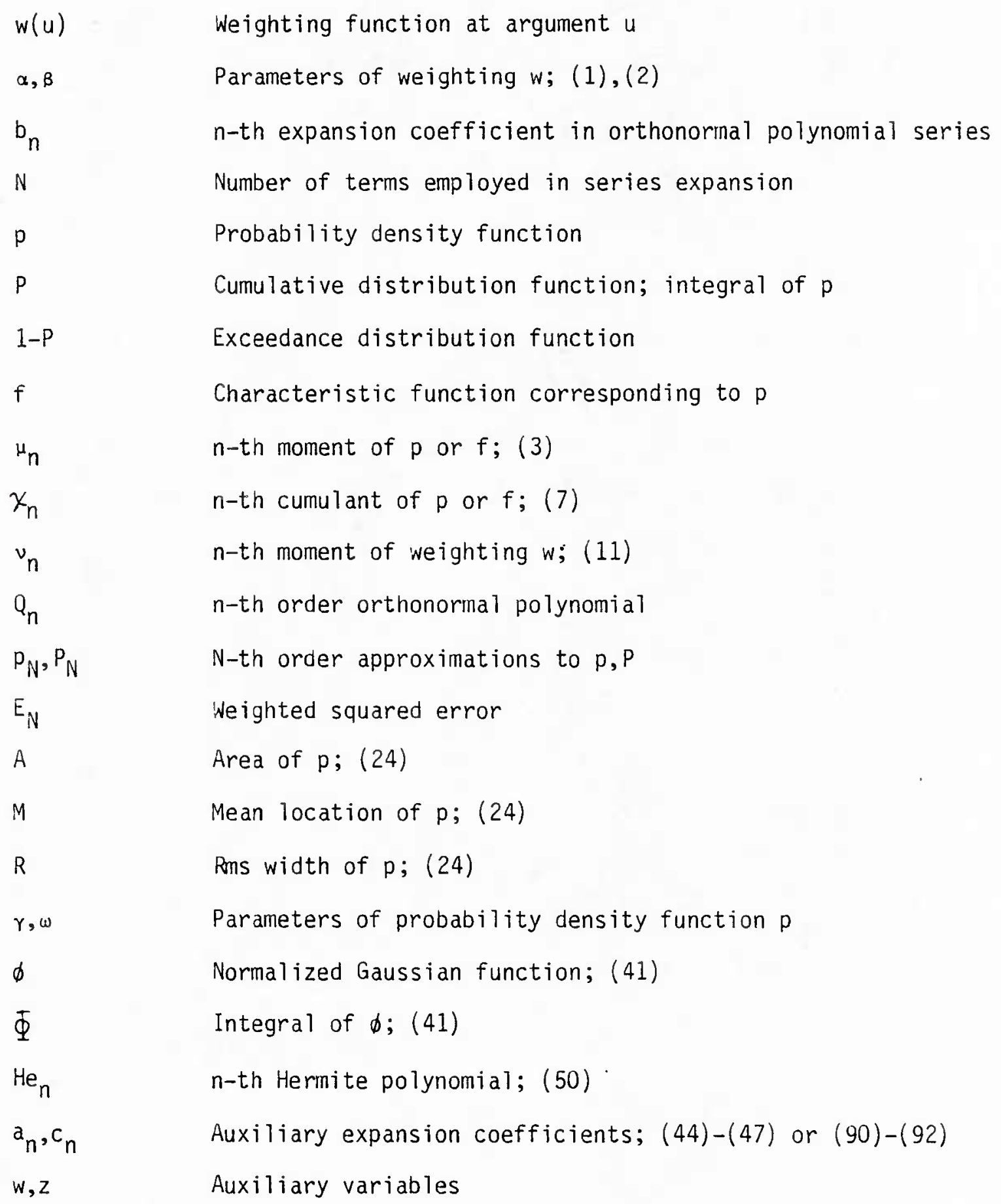


TR 7377

LIST OF SYMBOLS (Cont'd)

\begin{tabular}{|c|c|}
\hline$\dot{x}_{n}$ & Normalized cumulants; (62) \\
\hline$\hat{\mu}_{n}$ & Normalized moments; (69) \\
\hline$\tilde{\mu}_{n}$ & Alternative normalized moments; (118) \\
\hline$\hat{H e}_{n}$ & n-th normalized Hermite polynomial; (68) \\
\hline$H i_{n}$ & n-th modified Hermite polynomial; (74) \\
\hline$\hat{H} i_{n}$ & n-th normalized modified Hermite polynomial; (80) \\
\hline$L_{n}^{(\alpha)}$ & n-th generalized Laguerre polynomial; (96) \\
\hline${ }_{1} F_{1}$ & Conf luent hypergeometric function \\
\hline$d_{m}$ & Auxiliary variables in (112) \\
\hline$R C$ & Recursively via Cumulants \\
\hline DM & Directly via Moments \\
\hline RM & Recursively via Moments \\
\hline$\jmath_{, \theta} \theta$ & Parameters of $p$ in (141) \\
\hline$Q_{v}$ & Generalized Q-function; (165) \\
\hline$P_{0}$ & Area of impulse at origin of $p$, for shot noise \\
\hline$F$ & Hypergeometric function ${ }_{2} \mathrm{~F}_{1}$ \\
\hline Env & Envelope function \\
\hline$E_{n}(u ; p)$ & Estimated error of $p_{n} ;(178)$ or (183) \\
\hline$E_{n}(u ; P)$ & Estimated error of $P_{n} ;(180)$ or $(185)$ \\
\hline
\end{tabular}


EVALUATION OF DENSITIES AND DISTRIBUTIONS VIA HERMITE AND GENERALIZED LAGUERRE SERIES EMPLOYING HIGH-ORDER EXPANSION COEFFICIENTS DETERMINED RECURSIVELY VIA MOMENTS OR CUMULANTS

\author{
INTRODUCTION
}

'In the theoretical anaiysis of performance of some systems with nonlinearities and/or memory, it often happens that the only statistics about the decision (or output) random variable of interest that can be easily found are the moments, or in other cases, the cumulants. Explicit relations for the low-order expansion coefficients in Edgeworth or Gram-Charlier series are available in terms of the available moments or cumulants [1, pp. 172 and 191], [2, pp. 223 and 226], [3, pp. 157 and 159]. However, for higher-order moments and cumulants, these explicit nonrecursive relations are very tedious to derive, become extremely lengthy, and are not practical to use.

We will address the problem of obtaining accurate high-order series expansion approximations of the probability density function and cumulative distribution function of a random variable of interest, in terms of the available moments or cumulants of that random variable. The necessity of being able to approximate probability density functions and cumulative distribution functions from knowledge of either the moments or the cumulants, is that some physical problems have these particular statistics as natural and convenient starting points. For example, if a physical processor sums together a number of independent Rician random variates, the characteristic function and cumulants of the individual random variables or their sum are not available in any useful analytic form; however, the high-order moments of an 
TR 7377

individual Rician variate can be easily and accurately evaluated by recurrence, and thereby the moments of the sum can be obtained. Conversely, for shot noise with random amplitude and duration modulation, the probability density function is not readily available, whereas the characteristic function is, and the cumulants are simple to evaluate [4, appendix C].

The particular series expansions we employ are based on the two special classes of weighting functions

$$
w(u)=\frac{1}{(2 \pi)^{1 / 2} \beta} \exp \left(-\frac{(u-\alpha)^{2}}{2 \beta^{2}}\right) \text { for a11 } u \quad \text { Hermite }
$$

and

$$
w(u)=\frac{u^{\alpha} \exp (-u / \beta)}{\beta^{\alpha^{+}} \Gamma(\alpha+1)} \text { for } u>0 \quad \text { generalized Laguerre. }
$$

The orthonormal polynomials associated with these weightings are directly related to the Hermite and generalized Laguerre polynomials, respectively $[5,22.2 .15$ and 22.2.13]. The weightings each have two free parameters, $\alpha$ and $B$, which can be manipulated to advantage in obtaining finite (high-order) series expansions which well approximate a given (unknown) probability density function and cumulative distribution function.

The question of when a set of moments uniquely determines the probability density function is a difficult one; see, for example, [3, pp. 109-112 and 179]. Also, the convergence of the series is very involved [2, pp. 223 and 258], [3, pp. 161-163]. But, even if the series is divergent, use of a limited number of expansion coefficients often gives a satisfactory approximation to the desired probability density function $[3, p .167]$. We 
presume here that the moments do uniquely determine the probability density function and are buoyed in that respect by the comment [3, p. 87] that most distributions in statistical practice do possess this property.

The main idea in the series expansion approach here is not necessarily to get as many terms as possible, but rather to get as rapid convergence as possible of the series. If a particular choice of weighting parameters $\alpha$ and $\beta$ results in sufficiently small expansion coefficients, say, at order 10 , this is better than another choice of $\alpha$ and $\beta$ where 20 or 30 terms are required for the same size coefficients. In fact, if $\alpha$ and $\beta$ could be chosen such that the series terminated (zero coefficients) after a few terms, that would be ideal; however, this is not the case, and in fact, the choice of $\alpha$ and $\beta$ requires some trial-and-error to achieve rapidly decreasing coefficients.

The expansion coefficients of a given probability density function, in an orthonormal set of Hermite or generalized Laguerre polynomials, are denoted by $\left\{b_{n}\right\}_{0}^{N}$, where $N$ is the number of available or known moments or cumulants. Very often, the choice of $\alpha$ and $\beta$ in (1) or (2) has been made such that $b_{1}=0$ and $b_{2}=0$, for purposes of analytic simplicity and for hopeful early termination of the series; see for example [1, pp. 171 and 191], $[2$, p. 223], [3, p. 159]. However, it will be demonstrated that this is generally not the best choice, and that more rapidly decaying coefficients can be achieved by other (mismatched) values of $\alpha$ and $\beta$, which must be searched for numerically; this possibility is also mentioned in [3, p. 164]. In fact, an example will be given which illustrates that the choice of parameters $\alpha$ and $\beta$ to make expansion coefficients $b_{1}$ and $b_{2}$ zero, can in fact, lead to a divergent Hermite series. 
Depending on the available information about the probability density function, i.e., moments or cumulants, a variety of methods will be given for determining the expansion coefficients $\left\{b_{n}\right\}$. In particular, for both the Hermite and generalized Laguerre series, we can get the coefficients by three different procedures:
(a) recursively via cumulants,
(b) directly via moments,
(c) recursively via moments.

The reason for having these alternatives is that the calculation of expansion coefficients $\left\{b_{n}\right\}$ for high-order $n$ invariably runs into large round-off error. In order to reduce this round-off error, the amount of number-crunching on the computer should be minimized, and any spurious transformations between moments and cumulants should be avoided if possible. Thus it is desireable to have techniques which can accomplish the desired goal of evaluating expansion coefficients $\left\{b_{n}\right\}$ as directly as possible from the available information. The use of different alternatives also enables comparisons of the computed expansion coefficients and thereby furnishes quantitative assessment of the amount of round-off error. Recursive inter-relationships between moments, central moments, and cumulants are given in [6], including cases of two dependent random variables. 
FUNDAMENTAL EQUATIONS

DEF INITION OF STATISTICS

Suppose a function $\mathrm{p}$ has known moments*

$$
\mu_{n}=\int d u u^{n} p(u) \quad \text { for } 0 \leq n \leq N .
$$

The function $p$ need not have unit area, i.e., $\mu_{0} \neq 1$ is allowed, and $p$ can become negative at some arguments $u$. Nevertheless, for convenience, and since most of our applications are to random variables, we shall refer to $p$ as a probability density function, and to its running integral

$$
P(u)=\int_{-\infty}^{u} d t p(t)
$$

as a cumulative distribution function. We shall presume that $\mu_{0}>0$ in all cases.

The characteristic function corresponding to probability density function $p$ is the Fourier transform

$$
f(i \xi)=\int d u \exp (i \xi u) p(u) \text {. }
$$

When $f$ is expanded in a power series, the result is

$$
f(i \xi)=\sum_{n=0}^{\infty} \mu_{n}(i \xi)^{n} / n \text { ! }
$$

* Integrals without limits are over the range of nonzero integrand. 
TR 7377

in terms of the moments in (3). Alternatively, if $\ln f$ is expanded in a power series,

$$
\ln f(i \xi)=\sum_{n=0}^{\infty} \chi_{n}(i \xi)^{n} / n !
$$

where the quantities $\left\{\chi_{n}\right\}$ are the cumulants of $p$ or $f$. Observe that generally, to the lowest three orders,

$$
\begin{aligned}
& \chi_{0}=\ln f(0)=\ln \mu_{0} \neq 0, \\
& \chi_{1}=\frac{\mu_{1}}{\mu_{0}}, \\
& \chi_{2}=\frac{\mu_{2}}{\mu_{0}}-\left(\frac{\mu_{1}}{\mu_{0}}\right)^{2} .
\end{aligned}
$$

The available information on probability density function $p$ will be either

$$
\text { moments }\left\{\mu_{n}\right\}_{0}^{N} \text { or cumulants }\left\{\chi_{n}\right\}_{0}^{N}
$$

Whichever is available, we wish to get high-order accurate approximations to $\mathrm{p}$ and cumulative distribution function $P$ in (4); that is, values of $N$ in the order of 10 to 100 are of interest. 


\section{WEIGHTING FUNCTION PROPERTIES}

We select a nonnegative weighting function $w$ such that

$$
w(u)>0 \text { at least where } p(u) \neq 0 \text {. }
$$

We also disallow any impulses in $w$. The moments of weighting $w$ are defined analogousiy to (3) as

$$
v_{n}=\int d u u^{n} w(u) \text { for } n \geq 0 \text {; }
$$

it is presumed that these quantities can be evaluated for as large $n$ as required.

Suppose weighting $w$ has $r$ free parameters (plus a scaling parameter). It might then seem beneficial to choose them such that the moments of $w$ and $p$ are approximately equal,

$$
\nu_{n} \cong \mu_{n} \quad \text { for } 1 \leq n \leq r \quad\left(\text { plus } v_{0} \cong \mu_{0}\right) \text {, }
$$

for then the abscissa scales of $w$ and $p$ would tend to match. However, (12) will turn out to be not so desireable, and the choice of the $r$ weighting parameter values should be based on another criterion. The ordinate scale of $w$ is actually immaterial, since the expansion coefficients $\left\{b_{n}\right\}$ will absorb this scaling; so henceforth we presume that $v_{0}=1$ with no loss of generality. 
TR 7377

APPROXIMATION PROCEDURE

Let $Q_{n}$ be any $n$-th order polynomial, and approximate probability density function $p$ by function

$$
p_{N}(u) \equiv w(u) \sum_{n=0}^{N} b_{n} Q_{n}(u) \quad \text { where } w(u)>0
$$

where $\left\{b_{n}\right\}_{0}^{N}$ are the expansion coefficients. Define weighted squared error

$$
\begin{aligned}
E_{N} & =\int d u r(u)\left[p(u)-p_{N}(u)\right]^{2}= \\
& =\int d u r(u)\left[p(u)-w(u) \sum_{n=0}^{N} b_{n} Q_{n}(u)\right]^{2},
\end{aligned}
$$

where error-weighting $\gamma$ is nonnegative. If we minimize $E_{N}$ by choice of expansion coefficients $\left\{b_{n}\right\}_{0}^{N}$, there follows the set of linear equations

$\sum_{n=0}^{N} b_{n} \int d u r(u) w^{2}(u) Q_{k}(u) Q_{n}(u)=\int d u r(u) w(u) p(u) Q_{k}(u) \quad$ for $u \leq k \leq N$.

In order to use only the available information in (9) about $p$, the right-hand side of (15) must simplify according to the selection

$$
\gamma(u)=\frac{K}{w(u)} \text { where } w(u)>0 \text { (and arbitrary elsewhere). }
$$

Furthermore, since constant $K$ merely scales error $E_{N}$, and appears on both sides of (15), we can set $K=1$ without loss of generality. Then (14) becomes 


$$
E_{N}=\int d u w(u)\left[\frac{p(u)}{w(u)}-\sum_{n=0}^{N} b_{n} Q_{n}(u)\right]^{2} \quad \text { where } w(u)>0 \text {, }
$$

and (15) reduces to

$$
\sum_{n=0}^{N} b_{n} \int d u w(u) Q_{k}(u) Q_{n}(u)=\int d u p(u) Q_{k}(u) \text { for } 0 \leq k \leq N \text {. }
$$

In general, this is $\mathrm{N}+1$ simultaneous linear equations in the $\mathrm{N}+1$ unknowns $\left\{b_{n}\right\}_{0}^{N}$. The choice $Q_{k}(u)=u^{k}$ would lead to an apparently simple set of equations, when (11) and. (3) are used. However, a few numerical examples quickiy reveals that they are very $i 11$-conditioned, due to the character of the nondiagonal matrix with elements

$$
\int d u w(u) Q_{k}(u) Q_{n}(u) \quad \text { for } 0 \leq k, n \leq N
$$

that appears on the left-hand side of (18). In order to avoid the significant round-off error associated with solving such a system for large $N$, we choose $\left\{Q_{n}\right\}_{0}^{N}$ to be a set of orthonormal polynomials with respect to weighting w; i.e., (19) is 1 for $k=n$, and 0 otherwise. Also recall that $v_{0}=\int d u w(u)=1$ without loss of generality.

Equation (18) then reduces to an explicit relation for the expansion coefficients:

$$
b_{k}=\int d u p(u) Q_{k}(u) \quad \text { for } 0 \leq k \leq N \text {, }
$$

and (17) for the error becomes merely

$$
E_{N}=\int d u \frac{p^{2}(u)}{w(u)}-\sum_{n=0}^{N} b_{n}^{2}
$$


TR 7377

It will be presumed that the integral in (21) is finite; otherwise, the error would be infinite, which is a meaningless problem. This will put some restrictions on the parameter choices of weighting $w$, since this error integral depends on these parameters as well as on the given probability density function $p$. The sum of squares in (21) must then be bounded, and in fact affords a measure of the adequacy of approximation (13), by saturating (at an apriori unknown value) for large $\mathrm{N}$.

As $\mathrm{N}$ increases, the values of the lower-order expansion coefficients $\left\{b_{k}\right\}$ in (20) do not change. Therefore they only have to be computed once and do not have to be revised as more terms are added in series approximation (13), i.e., larger $N$.

EQUALITY OF PROBABILITY DENSITY FUNCTION MUMENTS

A very important property of expansion (13) is obtained as follows:

$$
\begin{aligned}
& \int d u Q_{k}(u) p_{N}(u)=\int d u Q_{k}(u) w(u) \sum_{n=0}^{N} b_{n} Q_{n}(u)= \\
& =b_{k}=\int d u Q_{k}(u) p(u) \text { for } 0 \leq k \leq N,
\end{aligned}
$$

where we used, in order, (13), the orthonormality of (19), and (20). But since $Q_{k}$ is a $k$-th order polynomial, relation (22) states that approximation $p_{N}$ has exactiy the same moments as given probability density function $p$, from order 0 through order $N$. This matching of moments between probability density functions $p_{N}$ and $p$ has been achieved regardless of the weighting $w$ and its particular parameter values. Furthermore, (22) holds independently of whether the weighting-moment equalities in (12) are satisfied or not. 
The cumulative distribution function corresponding to approximation $p_{N}$ is defined as

$$
P_{N}(u) \equiv \int_{-\infty}^{u} d t p_{N}(t)=\sum_{n=0}^{N} b_{n} \int_{-\infty}^{u} d t w(t) Q_{n}(t) .
$$

Its utility depends on getting closed forms and simple recursions for the general integral on the right-hand side.

PARAMETERS OF GIVEN PROBABILITY DENSITY FUNCTION $p$

The moments of $p$ were defined in (3). It is useful to define three important parameters of $p$ :

$$
\begin{aligned}
& \text { Area } A=\int d u p(u)=\mu_{0} \quad\left(\mu_{0}>0 \text {, but need not be } 1\right) \text {; } \\
& \text { Mean Location } M=\frac{\int d u u p(u)}{\int d u p(u)}=\frac{\mu_{1}}{\mu_{0}} ; \\
& \text { RMS width } R=\left[\frac{\int d u(u-M)^{2} p(u)}{\int d u p(u)}\right]^{1 / 2}=\left[\frac{\mu_{2}}{\mu_{0}}-\left(\frac{\mu_{1}}{\mu_{0}}\right)^{2}\right]^{1 / 2}
\end{aligned}
$$

(Conversely, $\mu_{0}=A, \mu_{1}=A M, \mu_{2}=A\left(M^{2}+R^{2}\right)$.) These parameters depend on the probability density function $p$ that we are trying to approximate and can be computed from the available information (9). They are useful for determining where the major concentration of $p(u)$ lies on the $u$-scale, and have obvious physical interpretations.

In terms of the cumulants of $p$ defined. in $(5)-(8)$, we have the alternative expressions 
TR 7377

$$
\mathrm{A}=\exp \left(\chi_{0}\right), \quad \mathrm{s}=\chi_{1}, \quad \mathrm{R}=\chi_{2}^{1 / 2},
$$

or conversely

$$
\chi_{0}=\ln \mu_{0}=\ln A, \quad \chi_{1}=\frac{\mu_{1}}{\mu_{0}}=M, \quad \chi_{2}=\frac{\mu_{2}}{\mu_{0}}-\left(\frac{\mu_{1}}{\mu_{0}}\right)^{2}=R^{2} .
$$

GENERAL RESULTS FOR THREE LOWEST-ORDER POLYNOMIALS $Q_{n}$

The weighting function $w$ and associated orthonormal polynomials satisfy the following equation:

$$
\int d u w(u) Q_{k}(u) Q_{n}(u)=\delta_{k n} \text {. }
$$

Also we have weighting moments

$$
\nu_{n}=\int d u u^{n} w(u), \quad \text { with } \nu_{0}=1 \text {. }
$$

It is then a straightforward matter to evaluate the three lowest-order orthonormal polynomials:

$$
\begin{aligned}
& Q_{0}(u)=1, \\
& Q_{1}(u)=\frac{1}{D_{1}}\left(u-v_{1}\right), \\
& Q_{2}(u)=\frac{1}{D_{2}}\left[u^{2}\left(v_{2}-v_{1}^{2}\right)-u\left(v_{3}-v_{2} v_{1}\right)+\left(v_{3} v_{1}-v_{2}^{2}\right)\right],
\end{aligned}
$$

where 


$$
\begin{aligned}
& D_{1}=\left(v_{2}-v_{1}^{2}\right)^{1 / 2}, \\
& D_{2}=\left(v_{2}-v_{1}^{2}\right)^{1 / 2}\left[\left(v_{4}-v_{2}^{2}\right)\left(v_{2}-v_{1}^{2}\right)-\left(v_{3}-v_{2} v_{1}\right)^{2}\right]^{1 / 2} .
\end{aligned}
$$

The general expansion coefficients in (20) then become

$$
\begin{aligned}
& b_{0}=\mu_{0}, \\
& b_{1}=\frac{1}{D_{1}}\left(\mu_{1}-v_{1} \mu_{0}\right), \\
& b_{2}=\frac{1}{D_{2}}\left[\mu_{2}\left(v_{2}-v_{1}^{2}\right)-\mu_{1}\left(v_{3}-v_{2} v_{1}\right)+\mu_{0}\left(v_{3} v_{1}-v_{2}^{2}\right)\right] .
\end{aligned}
$$

All these results above are general and make no presumption about weighting moment equalities such as (12).

SPECIAL CHOICES OF WEIGHTING PARAMETERS

Suppose that weighting $w$ has free parameters that can be varied so as to make the mean locations of $w$ and $p$ coincide (see (24)); that is,

$$
\text { let } \quad v_{1}=\frac{\mu_{1}}{\mu_{0}} \text {. }
$$

(The reason for the discrepancy with (12) is that we have set $v_{0}=1$ but have allowed $\left.\mu_{0} \neq 1.\right)$ Inspection of (31) gives the following:

$$
\text { then } b_{1}=0 \text { and } b_{2}=-\frac{\mu_{0}}{D_{2}}\left(\nu_{2}-\frac{\mu_{2}}{\mu_{0}}\right)\left(v_{2}-\frac{\mu_{1}^{2}}{\mu_{0}^{2}}\right) \text {. }
$$


TR 7377

Conversely, (31) shows that requiring $b_{1}=0$ forces the choice in (32A) for $v_{1}$. Thus equality of the first weighting moment $v_{1}$ of $w$ with the first (normalized) moment of probability density function $p$ implies (and is implied by) the vanishing of the first expansion coefficient $b_{1}$. This may or may not be a useful choice, but, whether adopted or not, has no bearing on the equality of probability density function moments already demonstrated in (22).

As a second special choice, suppose that weighting $w$ has enough free parameters that we can vary, so as to make the mean locations and rms widths of $w$ and $p$ coincide (see (24)); that is

$$
\text { let } v_{1}=\frac{\mu_{1}}{\mu_{0}} \text { and } v_{2}=\frac{\mu_{2}}{\mu_{0}} \text {. }
$$

(Again we have used $\left.v_{0}=1.\right)$ Manipulation of (31) yields the following conclusion:

$$
\text { then } b_{1}=0 \text { and } b_{2}=0
$$

Conversely, imposition of (33B) implies the results in (33A), as may be seen by reference to (31). (The apparent additional solution $v_{2}=\mu_{1}^{2} / \mu_{0}^{2}=v_{1}^{2}$ would yield an impulse for $w$ and is disallowed.) Thus equality of the first two weighting moments of $w$ with the first two (normalized) moments of probability density function p implies (and is implied by) the vanishing of the first two expansion coefficients $b_{1}$ and $b_{2}$. This common choice of weighting parameter values can be made if desired, but is not necessary (or recommended) for series approximations by orthonormal polynomials. The equality of probability density function moments in (22) will hold whether (33) is true or not. 
EXAMPLE OF DIVERGENT ERROR INTEGRAL FOR $b_{1}=0, b_{2}=0$

As a demonstration of what forcing expansion coefficients $b_{1}$ and $b_{2}$ equal to zero can do, consider probability density function

$$
p(u)=\frac{2 u^{\gamma} \exp \left(-u^{2} / \omega^{2}\right)}{\omega^{\gamma+1} \Gamma\left(\frac{\gamma^{+}}{2}\right)} \text { for } u>0 \quad(\gamma>-1, \omega>0)
$$

with moments

$$
\mu_{n}=\omega^{n} \frac{\Gamma\left(\frac{r^{+1+n}}{2}\right)}{\Gamma\left(\frac{y^{+1}}{2}\right)} .
$$

This class of probability density functions includes the one-sided Gaussian, Rayleigh, and Maxwell as special cases, for $\gamma=0,1,2$, respectively.

Consider also the Hermite weighting given in (1), which has moments (11) equal to

$$
v_{0}=1, \quad v_{1}=\alpha, \quad v_{2}=\alpha^{2}+\beta^{2} .
$$

If we now insist on property (33B), then (33A) yields

$$
\alpha=\omega \frac{\Gamma\left(\frac{\gamma}{2}+1\right)}{\Gamma\left(\frac{\gamma+1}{2}\right)}, \quad \beta^{2}=\omega^{2}\left[\frac{\gamma^{+1}}{2}-\frac{\Gamma^{2}\left(\frac{\gamma}{2}+1\right)}{\Gamma^{2}\left(\frac{\gamma+1}{2}\right)}\right] .
$$

But the leading integral in minimum error $E_{N}$ in (21) is convergent only if $p^{2}(u) / w(u)$ decays sufficiently rapid for large $u$. We have from (34) and (1), the dominant behavior

$$
p^{2}(u) / w(u) \propto \exp \left(-\frac{2 u^{2}}{\omega^{2}}+\frac{u^{2}}{2 \beta^{2}}\right) \text { for large positive } u,
$$

where $\propto$ denotes proportionality, but disregards the exact scale factor and subdominant behavior. Thus the integral in (21) is convergent only if 
TR 7377

$$
1<\frac{4 \beta^{2}}{\omega^{2}}=2(\gamma+1)-4 \frac{\Gamma^{2}\left(\frac{\gamma}{2}+1\right)}{\Gamma^{2}\left(\frac{\gamma+1}{2}\right)} .
$$

However, calculation of (39) reveals that this inequality is never satisfied for any value of $\gamma>-1$; the function on the right-hand side starts at 0 when $\gamma=-1$, and increases monotonically towards 1 as $\gamma \neq+\infty$, behaving 1 ike $1-1 /(4 \gamma)$ in this limit.

Thus expansion of probability density function (34) according to a Hermite weighting has an infinite error integral (21) (and perhaps a divergent series expansion) regardless of the values of $\gamma$ and $\omega$ in the true probability density function, if we insist on expansion coefficients $b_{1}=b_{2}=0$. Yet if we relax requirement (33B), and choose $B$ according to (39) such that $B>\omega / 2$, the error integral in (21) is certainly finite, regardless of $\alpha$.

However, making the error integral in (21) finite is not the whole story, in so far as realizing useful approximations to the probability density function or cumulative distribution function. An example of probability density function (34) was taken with $\gamma=3, \omega=1$. When $\alpha$ and $\beta$ were chosen according to (33) and (37) (giving $\beta=.48<.5=\omega / 2$ ), the expansion coefficients $\left\{b_{n}\right\}$ initially decreased to approximately $1 E-3$ at $n=40$ terms, and then diverged; yet a plot of the approximate exceedance distribution function obtained by a Hermite expansion overlaid the exact answer down to the 1E-16 level. On the other hand, when the weighting parameters in the Hermite expansion were chosen $a s^{\star} \alpha=0, \beta=.7>.5=\omega / 2$, giving $b_{1} \neq 0$ and

*This is example $B$ in a later section 
$b_{2} \neq 0$, the expansion coefficients $\left\{b_{n}\right\}$ decreased to the $1 E-4$ level at $n=70$ before round-off error became dominant; despite this apparent improvement in coefficient level, the approximate exceedance distribution function overlaid a plot of the exact result down to the $1 E-10$ probability level, which is several orders of magnitude worse than the above result. Thus emphasis on getting a convergent error integral in (21) may not a ways be desired.

For Hermite weighting (1) and the class of probability density functions which decay as $\exp \left(-u^{q}\right)$ as $u \rightarrow+\infty$, the error integral is always convergent if $q>2$, and always divergent if $q<2$. So an exponential probability density function, like $u^{\gamma} \exp (-u / \omega)$ for $u>0$, always yields a divergent error integral when expanded in a Hermite series.

For generalized Laguerre weighting (2), it is necessary to consider $u=0+$ and $u=+\infty$ separately. If probability density function $p$ behaves like $u^{\gamma}$ as $u \rightarrow 0^{+}$, then a finite error integral requires that we choose $\alpha<1+2 \gamma$. Coupled with the finite area restriction on weighting $w$, a range of values of $\alpha$ is allowed, namely, $-1<\alpha<1+2 \gamma$; this range always exists since $\gamma>-1$ is necessary for the probability density function itself to have finite area.

If also the probability density function behaves as $\exp (-u / \omega)$ as $u \rightarrow+\infty$, then a finite error integral with generalized Laguerre weighting requires that we choose $\beta>\omega / 2$. So the range of choice of $\beta$ is open on the large side, whereas that for $\alpha$ is a limited one, for this particular class of probability density functions. 
TR 7377

\section{HERMITE EXPANSION}

In this section, we will deal exclusively with weighting (1),

$$
W(u)=\frac{1}{\beta} \phi\left(\frac{u-\alpha}{\beta}\right) \text { for ali } u \quad(\beta>0),
$$

where

$$
\phi(x)=(2 \pi)^{-1 / 2} \exp \left(-x^{2} / 2\right), \quad \Phi(x)=\int_{-\infty}^{x} d t \phi(t) .
$$

This weighting has two free parameters, $\alpha$ and $\beta$, and moments

$$
v_{0}=1, \quad v_{1}=\alpha, \quad v_{2}=\alpha^{2}+\beta^{2} .
$$

If $v_{1}$ and $v_{2}$ are specified, the parameters must then satisfy $\alpha=v_{1}$,

$\beta=\left(v_{2}-v_{1}^{2}\right)^{1 / 2}$. However, we shall keep $\alpha$ and $\beta$ general and unspecified.

PROPERTIES OF POLYNOMIALS AND EXPANSIONS

The orthonomal polynomials associated with weighting (40) are the Hermite polynomials $[5,22.1 .2$ and 22.2.15]

$$
Q_{n}(u)=H_{n}\left(\frac{\mu-\alpha}{\beta}\right)(n !)^{-1 / 2} \text { for } n \geq 0 \text {. }
$$

The expansion coefficients are given by (20) as

$$
b_{n}=\int d u p(u) Q_{n}(u)=(n !)^{-1 / 2} c_{n} \text { for } n \geq 0,
$$


where we define

$$
c_{n}=\int d u p(u) H_{n}\left(\frac{u-\alpha}{\beta}\right) \text { for } n \geq 0 .
$$

The approximate probability density function then follows from (13) in the form

$$
p_{N}(u)=w(u) \sum_{n=0}^{N} b_{n} Q_{n}(u)=\frac{1}{\beta} \phi\left(\frac{u-\alpha}{\beta}\right) \sum_{n=0}^{N} a_{n} H e_{n}\left(\frac{u-\alpha}{\beta}\right) \text {, }
$$

where we used $(40),(43),(44)$, and defined

$$
(n !)^{1 / 2} a_{n}=b_{n}=(n !)^{-1 / 2} c_{n} \text { for } n \geq 0 \text {. }
$$

These three different coefficients in (44)-(47) are introduced for convenience in further equation manipulations. Expansion coefficient $b_{n}$ is the geometric mean of auxiliary coefficients $a_{n}$ and $c_{n}$ (with polarity). Expansion (46) is also called a Gram-Charlier series of type $A[2, p .222]$, $[3$, p. 156].

The approximate cumulative distribution function corresponding to (46) is

$$
\begin{aligned}
& P_{N}(u)=\int_{-\infty}^{u} d t p_{N}(t)=\sum_{n=0}^{N} a_{n} \int_{-\infty}^{u} \frac{d t}{\beta} \phi\left(\frac{t-\alpha}{\beta}\right) H e_{n}\left(\frac{t-\alpha}{\beta}\right)= \\
& =\sum_{n=0}^{N} a_{n} \int_{-\infty}^{T} d x \phi(x) H e_{n}(x)=a_{0} \Phi(T)-\phi(T) \sum_{n=1}^{N} a_{n} H e_{n-1}(T),
\end{aligned}
$$

where

$$
T=\frac{u-\alpha}{\beta}
$$

and we used (41) and $[5,22.11 .8]$. 
The Hermite polynomials $\left\{\mathrm{He}_{n}\right\}$ satisfy the recurrence $[5,22.7 .14]$

$$
H e_{n}(x)=x H e_{n-1}(x)-(n-1) H e_{n-2}(x) \text { for } n \geq 2 \text {, }
$$

with starting values $\mathrm{He}_{0}(x)=1, \mathrm{He}_{1}(x)=x[5,22.3 .11]$. The highestorder term in $\mathrm{He}_{n}(x)$ is $x^{n}$, with coefficient $1[5,22.1 .2$ and 22.3.11]. The magnitude of the term multiplying $b_{n}$ in (46) has an envelope that decays approximately as $n^{-1 / 4}$ with $n$, regardless of argument $u$. This may be seen by using (47) and (49) to get

$$
\begin{array}{r}
a_{n} H e_{n}(T)=b_{n}(n !)^{-1 / 2} H e_{n}(T) \propto b_{n}\left(n^{n+1 / 2} e^{-n}\right)^{-1 / 2}(n / e)^{n / 2}=b_{n} n^{-1 / 4} \\
\text { as } n \rightarrow+\infty \text {, for a } 17 T,
\end{array}
$$

where we also used $[5,6.1 .39$ and 22.5 .18$]$ and $[7,8.22 .8]$. Here, $\alpha$ denotes proportionality and we have taken the magnitude of the terms; the exact scale factor of proportionality will be presented in a later section where the errors of the approximations are estimated. So if $b_{n}$ were to decay faster than $n^{-3 / 4}$, the probability density function series in (46) would converge absolutely.

Conditions are better for the cumulative distribution function series in (48); namely, based on the above result, there follows (for the envelope)

$$
\begin{aligned}
& a_{n} H e_{n-1}(T)=b_{n}(n !)^{-1 / 2} H e_{n-1}(T)=b_{n} n^{-1 / 2}[(n-1) !]^{-1 / 2} H e_{n-1}(T)= \\
& \mathcal{L} b_{n} n^{-1 / 2} n^{-1 / 4}=b_{n} n^{-3 / 4} \text { as } n \rightarrow+\infty, \text { for all T. }
\end{aligned}
$$

Thus if $b_{n}$ decays faster than $n^{-1 / 4}$, the cumulative distribution function series converges absolutely. Furthermore, if the leading error integral in 
(21) is finite, the sum of $b_{n}^{2}$ must be finite, meaning that $b_{n}$ must decay faster than $n^{-1 / 2}$. So we can conclude that if the error integral is finite, the Hermite series for the cumulative distribution function in (48)

converges. (Notice that this particular decay $n^{-1 / 2}$ of $b_{n}$ is not

sufficientiy fast to make the same conclusion about the Hermite series for the probability density function in (46).) The above are sufficient conditions on expansion coefficients $\left\{b_{n}\right\}$, and are not necessary.

EXPANSION OF CHARACTERISTIC FUNCTION $f$

The coefficients $a_{n}$ and $c_{n}$ were defined in (45) and (47). Then the sum

$$
\begin{aligned}
& \sum_{n=0}^{\infty} a_{n} w^{n}=\sum_{n=0}^{\infty} \frac{1}{n !} c_{n} w^{n}=\sum_{n=0}^{\infty} \frac{w^{n}}{n !} \int d u p(u) H e_{n}\left(\frac{u-\alpha}{\beta}\right)= \\
& =\int d u p(u) \sum_{n=0}^{\infty} \frac{w^{n}}{n !} H e_{n}\left(\frac{u-\alpha}{\beta}\right)=\int d u p(u) \exp \left(\frac{u-\alpha}{\beta} w-\frac{1}{2} w^{2}\right)= \\
& =\exp \left(-\frac{1}{2} w^{2}-\frac{\alpha}{\beta} w\right) f\left(\frac{w}{\beta}\right),
\end{aligned}
$$

where $f$ is the characteristic function, and where we used $(45),[5,22.5 .19$ and 22.9.17], and (5). Letting $w=\beta Z$, we have

$$
f(z) \exp \left(-\alpha z-\frac{1}{2} \beta^{2} z^{2}\right)=\sum_{n=0}^{\infty} a_{n}(\beta z)^{n}=\sum_{n=0}^{\infty} \frac{1}{n !} c_{n}(\beta z)^{n} \text {. }
$$

Thus $\left\{a_{n}\right\}$ and $\left\{c_{n}\right\}$ are the coefficients in these power series expansions of the function $f(z) \exp \left(-\alpha z-\beta^{2} z^{2} / 2\right)$, where $f$ is the characteristic function corresponding to probability density function $p$, and $\alpha$ and $\beta$ are arbitrary. A special case of (52) is given in $[2,17.6 .10]$. 
TR 7377

Collecting (46) and (52) together for comparison, and assuming that

$p_{n} \rightarrow p$ as $N \rightarrow+\infty$, we have

$$
\begin{aligned}
& p(u)=\frac{1}{\beta} \phi\left(\frac{u-\alpha}{\beta}\right) \sum_{n=0}^{\infty} a_{n} H e_{n}\left(\frac{u-\alpha}{\beta}\right), \\
& f(i \xi)=\exp \left(i \alpha \xi-\frac{1}{2} \beta^{2} \xi^{2}\right) \sum_{n=0}^{\infty} a_{n}(i \beta \xi)^{n} .
\end{aligned}
$$

Thus expansion of probability density function $p$ in an infinite Hermite series is equivalent to an expansion of a modified form of the characteristic function in a power series, according to (53). Equations (51)-(53) will serve as very convenient starting points for the derivation of several alternative recurrences for the expansion coefficients $\left\{a_{n}\right\}$. Notice that weighting parameters $\alpha$ and $\beta$ are completely unrestricted in (52) and (53), except that B $>0$.

An analogous result holds for $\mathrm{N}$ finite, but must be derived in a different fashion, because we no longer can use infinite sum $[5,22.9 .17]$. Define the Fourier transform of (46) as the $\mathrm{N}$-th order approximation to the characteristic function:

$$
\begin{gathered}
f_{N}(i \xi)=\int d u \exp (i \xi u) p_{N}(u)= \\
=\sum_{n=0}^{N} a_{n} \int d u \exp (i \xi u) \frac{1}{\beta} \phi\left(\frac{u-\alpha}{\beta}\right) H e_{n}\left(\frac{u-\alpha}{\beta}\right)= \\
=\sum_{n=0}^{N} a_{n} \int d t \exp (i \xi \alpha+i \xi \beta t) \phi(t) H e_{n}(t)= \\
=\exp (i \alpha \xi) \sum_{n=0}^{N} a_{n} \int d t \exp (i \beta \xi t)\left(-\frac{d}{d t}\right)^{n} \phi(t)=
\end{gathered}
$$




$$
\begin{aligned}
& =\exp (i \alpha \xi) \sum_{n=0}^{N} a_{n}(i \beta \xi)^{n} \int d t \exp (i \beta \xi t) \phi(t)= \\
& =\exp \left(i \alpha \xi+\frac{1}{2} \beta^{2}(i \xi)^{2}\right) \sum_{n=0}^{N} a_{n}(i \beta \xi)^{n},
\end{aligned}
$$

where we used $[5,22.11 .8]$ in line 4 , and repeated integration by parts in line 5. This result is the leading $N$ terms of (53). As a by-product of this derivation, we have

$$
\int d t \exp (z t) \phi(t) H e_{n}(t)=\exp \left(\frac{1}{2} z^{2}\right) z^{n} .
$$

COEFFICIENTS RECURSIVELY VIA CUMULANTS

We are now in a position to obtain some useful recursive relations for the expansion coefficients $\left\{a_{n}\right\}$ in $(51)-(54)$. The first one is obtained by taking the $\ln$ of $(51)$ :

$$
\ln f\left(\frac{w}{\beta}\right)-\frac{\alpha}{\beta} w-\frac{1}{2} w^{2}=\ln \left\{\sum_{n=0}^{\infty} a_{n} w^{n}\right\} .
$$

Then using (7) and identifying the right-hand side of (56) as a new power series, we have

$$
\sum_{n=0}^{\infty} \frac{1}{n !} \chi_{n}\left(\frac{w}{\beta}\right)^{n}-\frac{\alpha}{\beta} w-\frac{1}{2} w^{2}=\sum_{n=0}^{\infty} n_{n} w^{n} .
$$


TR 7377

There follows immediately

$$
n_{n}=\left\{\begin{array}{ll}
\frac{\chi_{n}}{n ! \beta^{n}} & \text { for } n \neq 1,2 \\
\frac{\chi_{1}-\alpha}{\beta} & \text { for } n=1 \\
\frac{1}{2}\left(\frac{\chi_{2}}{\beta^{2}}-1\right) & \text { for } n=2
\end{array}\right\} \text {. }
$$

But equality of the right-hand sides of (56) and (57) also requires that

$$
\sum_{n=0}^{\infty} a_{n} w^{n}=\exp \left\{\sum_{n=0}^{\infty} n_{n} w^{n}\right\}
$$

It is shown in appendix $A$ that a recursive solution to (59) for the $\left\{a_{n}\right\}$ is given by

$$
a_{n}=\frac{1}{n} \sum_{m=1}^{n} m h_{m} a_{n-m} \text { for } n \geq 1, \quad a_{0}=\exp \left(h_{0}\right) .
$$

Then eliminating $\left\{h_{m}\right\}$ by means of (58),

$$
\begin{gathered}
a_{n}=\frac{1}{n}\left[\left(\frac{\chi_{1}}{\beta}-\frac{\alpha}{\beta}\right) a_{n-1}+\left(\frac{\chi_{2}}{\beta^{2}}-1\right) a_{n-2}+\sum_{m=3}^{n} \frac{\chi_{m}}{(m-1) ! \beta^{m}} a_{n-m}\right] \text { for } n \geq 1 \\
a_{0}=\exp \left(\chi_{0}\right)
\end{gathered}
$$

where $a_{n} \equiv 0$ for $n<0$, and the sum is zero for $n<3$.

Now define normalized cumulants (excluding $n=0$ ) according to

$$
\hat{\chi}_{n}=\frac{\chi_{n}}{(n-1) ! \beta^{n}} \text { for } n \geq 1
$$


TR 7377

Then (61) becomes

$$
\begin{array}{r}
a_{n}=\frac{1}{n}\left[\left(\hat{x}_{1}-\frac{\alpha}{\beta}\right) a_{n-1}+\left(\hat{x}_{2}-1\right) a_{n-2}+\sum_{m=3}^{n} \hat{\chi}_{m} a_{n-m}\right] \quad \text { for } n \geq 1, \\
a_{0}=\exp \left(x_{0}\right) .
\end{array}
$$

This convolution is the desired recursion for expansion coefficients $\left\{a_{n}\right\}$ via cumulants.

As particular cases, we have

$$
a_{1}=\frac{\chi_{1}-\alpha}{\beta} a_{0}, \quad a_{2}=\frac{1}{2}\left[\left(\frac{\chi_{1}-\alpha}{\beta}\right)^{2}+\frac{\chi_{2}}{\beta^{2}}-1\right] a_{0} .
$$

Parameters $\alpha$ and $\beta(>0)$ are completely arbitrary in the above three equations, and $\left\{\chi_{n}\right\}_{0}^{N}$ are the available cumulants of the probability density function under consideration.

Observe that if we choose $\alpha=\chi_{1}=M$ and $\beta=\chi_{2}^{1 / 2}=R($ see $(24)-(25))$, which is a very common choice, we have $a_{1}=0$ and $a_{2}=0$; this is a special case of the general property (33) stated earlier. This special choice of $\alpha$ and $\beta$ corresponds to choosing the mean location and rms width of Hermite weighting (40) identical to those same parameters of the given probability density function. There then also follows, in this special case,

$$
\begin{gathered}
a_{3}=\frac{1}{3} \hat{\chi}_{3} a_{0}, \quad a_{4}=\frac{1}{4} \hat{\chi}_{4} a_{0}, \quad a_{5}=\frac{1}{5} \hat{\chi}_{5} a_{0}, \\
a_{n}=\frac{1}{n}\left[\hat{\chi}_{n} a_{0}+\sum_{m=3}^{n-3} \hat{\chi}_{m} a_{n-m}\right] \text { for } n \geq 6 \text { when } \alpha=\chi_{1}, \quad \beta=\chi_{2}^{1 / 2} .
\end{gathered}
$$


TR 7377

COEFF ICIENTS DIRECTLY VIA MOMENTS

Before we begin this derivation, we present the following useful expansion $[5,22.9 .17$ and 22.5.19]:

$$
\exp \left(-\frac{1}{2} y^{2}+x y\right)=\sum_{n=0}^{\infty} \frac{1}{n !} H_{n}(x) y^{n} \text {. }
$$

We now again refer to (51) and expand the terms as follows:

$$
\begin{aligned}
& \sum_{n=0}^{\infty} a_{n} w^{n}=\exp \left(-\frac{1}{2} w^{2}-\frac{\alpha}{\beta} w\right) f\left(\frac{w}{\beta}\right)= \\
& =\sum_{k=0}^{\infty} \frac{1}{k !} H e_{k}\left(-\frac{\alpha}{\beta}\right) w^{k} \sum_{m=0}^{\infty} \frac{1}{m !} \mu_{m}\left(\frac{w}{\beta}\right)^{m},
\end{aligned}
$$

where we utilized (65) and (6). Equating coefficients of $w^{n}$ on both sides of this equation, we have

$$
a_{n}=\sum_{k=0}^{n} \frac{1}{k !} H e_{k}\left(-\frac{\alpha}{\beta}\right) \frac{\mu_{n-k}}{(n-k) ! \beta^{n-k}} \text { for } n \geq 0 \text {. }
$$

We now define, for convenience, the normalized Hermite polynomials

$$
\hat{H e}_{n}(x)=\frac{1}{n !} H e_{n}(x) \text { for } n \geq 0 \text {, }
$$

and the normalized moments

$$
\hat{\mu}_{n}=\frac{\mu_{n}}{n ! \beta^{n}} \text { for } n \geq 0
$$

(Notice the difference with the definition of the normalized cumulants (62).) Then (67) becomes 


$$
a_{n}=\sum_{k=0}^{n} \hat{H}_{k}\left(-\frac{\alpha}{\beta}\right) \hat{\mu}_{n-k} \text { for } n \geq 0 \text {, }
$$

which gives expansion coefficients $\left\{a_{n}\right\}$ directly in terms of the (normalized) moments of the given probability density function. The recurrence in (50) can be used to generate the Hermite factors needed in convolution (70). Parameters $\alpha$ and $\beta(>0)$ of weighting (40) are arbitrary. $\hat{H}_{e_{0}}(x)=1, \quad \hat{H}_{e_{1}}(x)=x, \hat{H}_{e_{n}}(x)=\frac{1}{n}\left[x \hat{H}_{e_{n-1}}(x)-\hat{H}_{e_{n-2}}(x)\right]$ for $n \geq 2$.

As particular cases, we have

$$
a_{0}=\mu_{0}, \quad a_{1}=\frac{\mu_{1}-\alpha \mu_{0}}{\beta}, \quad \alpha_{2}=\frac{\mu_{2}-2 \alpha \mu_{1}+\left(\alpha^{2}-\beta^{2}\right) \mu_{0}}{2 \beta^{2}} .
$$

These agree with (64) which utilized cumulants. If we make the special choice of $\alpha=\mu_{1} / \mu_{0}$ and $\beta^{2}=\mu_{2} / \mu_{0}-\left(\mu_{1} / \mu_{0}\right)^{2}$, then $a_{1}=0$ and $a_{2}=0$.

An alternative more direct derivation of (67) is possible: from (47), $(45),(B-3)$ in appendix $B$, and (3),

$$
\begin{aligned}
a_{n} & =\frac{1}{n !} c_{n}=\frac{1}{n !} \int d u p(u) H e_{n}\left(\frac{u-\alpha}{\beta}\right)= \\
& =\frac{1}{n !} \int d u p(u) \sum_{k=0}^{n}\left(\begin{array}{l}
n \\
k
\end{array}\right) H e_{k}\left(-\frac{\alpha}{\beta}\right)\left(\frac{u}{\beta}\right)^{n-k}= \\
& =\sum_{k=0}^{n} \frac{1}{k !} H e_{k}\left(-\frac{\alpha}{\beta}\right) \frac{\mu_{n-k}}{(n-k) ! \beta^{n-k}} \text { for } n \geq 0 .
\end{aligned}
$$


TR 7377

COEFF ICIENTS RECURS IVELY VIA MOMENTS

Before we begin this derivation, we replace $x \rightarrow-i x, y \rightarrow i y$ in (65) to get

$$
\exp \left(\frac{1}{2} y^{2}+x y\right)=\sum_{n=0}^{\infty} \frac{1}{n !} H e_{n}(-i x)(i y)^{n}=\sum_{n=0}^{\infty} \frac{1}{n !} H i_{n}(x) y^{n},
$$

where $\mathrm{Hi}_{n}(\mathrm{x})$ is a real $\mathrm{n}$-th order modified Hermite polynomial in $\mathrm{x}$ defined by

$$
H i_{n}(x)=i^{n} H_{n}(-i x) \text { for } n \geq 0 \text {. }
$$

The recursion for these polynomials follows immediately from (50) as

$$
H i_{n}(x)=x H i_{n-1}(x)+(n-1) H i_{n-2}(x) \text { for } n \geq 2 \text {, }
$$

with starting values $\mathrm{Hi}_{0}(x)=1, H i_{1}(x)=x$. The difference with $(50)$ is the polarity of the last term; thus for example, $\mathrm{Hi}_{2}(x)=x^{2}+1$, $\mathrm{Hi}_{3}(x)=x^{3}+3 x$, versus $\mathrm{He}_{2}(x)=x^{2}-1, \mathrm{He}_{3}(x)=x^{3}-3 x$.

We now rewrite (51) in the following form:

$$
f\left(\frac{w}{\beta}\right)=\exp \left(\frac{1}{2} w^{2}+\frac{\alpha}{\beta} w\right) \sum_{m=0}^{\infty} a_{m} w^{m} .
$$

Expanding in power series by means of (6) and (73),

$$
\sum_{n=0}^{\infty} \frac{1}{n !} \mu_{n}\left(\frac{w}{\beta}\right)^{n}=\sum_{k=0}^{\infty} \frac{1}{k !} H i_{k}\left(\frac{\alpha}{\beta}\right) w^{k} \sum_{m=0}^{\infty} a_{m} w^{m} \text {. }
$$

Equating coefficients of $w^{n}$, there follows

$$
\frac{\mu_{n}}{n ! \beta^{n}}=\sum_{k=0}^{n} \frac{1}{k !} H i_{k}\left(\frac{\alpha}{\beta}\right) a_{n-k} \text { for } n \geq 0 \text {, }
$$

or 


$$
\hat{\mu}_{n}=\sum_{k=0}^{n} \hat{H}_{k}\left(\frac{\alpha}{\beta}\right) a_{n-k}=a_{n}+\sum_{k=1}^{n} \hat{H} i_{k}\left(\frac{\alpha}{\beta}\right) a_{n-k} \text { for } n \geq 0 \text {, }
$$

where we have used normalized moments (69), and defined the normalized modified Hermite polynomials

$$
\hat{H} i_{n}(x)=\frac{1}{n !} H i_{n}(x) \text { for } n \geq 0 \text {. }
$$

Finally, the desired recursion for expansion coefficients $\left\{a_{n}\right\}$ in terms of the moments follows as

$$
a_{n}=\hat{\mu}_{n}-\sum_{k=1}^{n} \hat{H}_{k}\left(\frac{\alpha}{\beta}\right) a_{n-k} \text { for } n \geq 0
$$

Parameters $\alpha$ and $\beta(>0)$ are arbitrary in (81) and (69).

SUMMARY

The approximations to the probability density function and cumulative distribution function are given by (46) and (48), respectively, where $\alpha$ and $\beta$ are arbitrary constants, except that $\beta>0$. The functions $\phi$ and $\Phi$ are defined in (41), while the Hermite polynomials $\left\{\mathrm{He}_{n}\right\}$ are available via (50). The expansion coefficients $\left\{a_{n}\right\}$ are given by the three alternatives (63), (70), (81), in terms of normalized cumulants (62), normalized moments (69), normalized Hermite polynomials (68), and normalized modified Hermite polynomials (80) and (74). Programs for all three alternative procedures for determining expansion coefficients $\left\{a_{n}\right\}$ are listed in an appendix. The basis for these relations is the characteristics function expansion in (51) $-(53)$. 
TR 7377

GENERAL IZED LAGUERRE EXPANS ION

This section will treat weighting (2), nameiy,

$$
w(u)=\frac{u^{\alpha} \exp (-u / \beta)}{\beta^{\alpha^{+}} \Gamma\left(\alpha^{+1}\right)} \text { for } u>0 \quad(\alpha>-1, \beta>0) \text {. }
$$

This weighting is a special case of the three-parameter weighting

$$
\frac{(u-\gamma)^{\alpha} \exp \left(-\frac{u-\gamma}{\beta}\right)}{\beta^{\alpha^{+1}} \Gamma\left(\alpha^{+} 1\right)} \text { for } u>\gamma \text {, }
$$

which is the most general scaled linear shift of the generalized Laguerre weighting $[5,22.2 .12]$

$$
x^{\alpha} \exp (-x) \text { for } x>0
$$

We will consider only $\gamma=0$ here. For a probability density function $p_{0}(u)$ which is known to be nonzero only for $u>u_{0}$, we would consider the modified probability density function $p(u)=p_{0}\left(u+u_{0}\right)$, because then $p(u) \neq 0$ only for $u>0$, and the simpler weighting (82) would be directly applicable. This procedure is equivalent to choosing $r=u_{0}$ in the three-parameter weighting (83) above, and requires knowledge of $u_{0}$. We presume that $p(u) \neq 0$ only for $u>0$ henceforth in this section, and that any necessary shifting has already taken place.

Weighting (82) has two free parameters, $\alpha$ and $\beta$, and moments

$$
\nu_{n}=(\alpha+1)_{n} B^{n} \text { for } n \geq 0 \text {. }
$$


In particular,

$$
v_{0}=1, \quad v_{1}=(\alpha+1)_{\beta}, \quad v_{2}=(\alpha+2)(\alpha+1)_{\beta}^{2} .
$$

If $v_{1}$ and $v_{2}$ are specified, then the parameters must satisfy

$$
\alpha=\frac{v_{1}^{2}}{v_{2}-v_{1}^{2}}-1, \quad \beta=\frac{v_{2}-v_{1}^{2}}{v_{1}} .
$$

However, we shall keep $\alpha$ and $\beta$ general and unspecified except for the conditions in (82).

\section{PROPERTIES OF POLYNOMIALS AND EXPANSIONS}

The orthonormal polynomials associated with weighting (82) are the generalized Laguerre polynomials $[5,22.1 .2$ and 22.2.12]

$$
Q_{n}(u)=L_{n}^{(\alpha)}\left(\frac{u}{\beta}\right)\left(\frac{n !}{(\alpha+1)_{n}}\right)^{1 / 2} \text { for } n \geq 0, u>0 .
$$

The expansion coefficients are given by (20) as

$$
b_{n}=\int_{0}^{\infty} d u p(u) a_{n}(u)=\left(\frac{n !}{(\alpha+1)_{n}}\right)^{1 / 2} c_{n} \text { for } n \geq 0,
$$

where we define

$$
c_{n}=\int_{0}^{\infty} d u p(u) L_{n}^{(\alpha)}\left(\frac{u}{\beta}\right) \text { for } n \geq 0 .
$$

The approximate probability density function follows from (13) according to 
TR 7377

$$
\begin{aligned}
& p_{N}(u)=w(u) \quad \sum_{n=0}^{N} b_{n} Q_{n}(u)= \\
& =\frac{u^{\alpha} \exp (-u / \beta)}{\beta^{+1} \Gamma\left(\alpha^{+}\right)} \sum_{n=0}^{N} a_{n} L_{n}^{(\alpha)}\left(\frac{u}{\beta}\right) \text { for } u>0,
\end{aligned}
$$

where we used (82), (88), (89), and defined

$$
\left(\frac{\left(\alpha^{+1}\right)_{n}}{n !}\right)^{1 / 2} a_{n}=b_{n}=\left(\frac{n !}{(\alpha+1)_{n}}\right)^{1 / 2} c_{n} \text { for } n \geq 0
$$

These three different coefficients in (89)-(92) are introduced for convenience in further equation manipulations. Expansion coefficient $b_{n}$ is the geometric mean of auxiliary coefficients $a_{n}$ and $c_{n}$ (with polarity).

The approximate cumulative distribution function corresponding to (91) is

$$
\begin{gathered}
P_{N}(u)=\int_{0}^{u} d t p_{N}(t)=\sum_{n=0}^{N} a_{n} \int_{0}^{u} d t \frac{t^{\alpha} \exp (-t / \beta)}{\beta^{\alpha+1} \Gamma(\alpha+1)} L_{n}^{(\alpha)}\left(\frac{t}{\beta}\right)= \\
=\frac{1}{\Gamma(\alpha+1)} \sum_{n=0}^{N} a_{n} I_{n}\left(\frac{u}{\beta}\right) \text { for } u>0,
\end{gathered}
$$

where we define

$$
I_{n}(y)=\int_{0}^{y} d x x^{\alpha} e^{-x} \underset{n}{L(\alpha)}(x) \text { for } n \geq 0, y>0 \text {. }
$$

These quantities are evaluated in appendix C; when substituted in (93), they yield

$$
P_{N}(u)=\frac{(u / \beta)^{\alpha^{+1}} \exp (-u / \beta)}{\Gamma\left(\alpha^{+1}\right)}\left[\frac{a_{0}}{\alpha^{+1}} F_{1}\left(1 ; \alpha+2 ; \frac{u}{\beta}\right)+\sum_{n=1}^{N} \frac{a_{n}}{n} L \frac{(\alpha+1)}{n-1}\left(\frac{u}{\beta}\right)\right] \text { for } u>0 \text {, }
$$

where ${ }_{1} F_{1}$ is the confluent hypergeometric function. 
The generalized Laguerre polynomials $\left\{\begin{array}{c}(\alpha) \\ n\end{array}\right\}$ satisfy the recurrence $[5,22.7 .12]$

$$
L_{n}^{(\alpha)}(x)=\frac{1}{n}\left[(\alpha-1+2 n-x) L_{n-1}^{(\alpha)}(x)-(\alpha-1+n) L_{n-2}^{(\alpha)}(x)\right] \text { for } n \geq 2,
$$

with starting values $\mathrm{L}_{0}^{(\alpha)}(x)=1, \mathrm{~L}_{1}^{(\alpha)}(x)=\alpha^{+} 1-x[5,22.4 .7]$. The highest order term in $L_{n}^{(\alpha)}(x)$ is $(-x)^{n} / n ![5,22.1 .2$ and 22.3.9]; this is distinctly different from the coefficient 1 for the Hermite polynomials. Yet the envelope decay with $n$ of the generalized Laguerre series for the probability density function and cumulative distribution function are identical to those of the Hermite series, for $u>0$. To prove this, use (91) and (92) to get

$$
\begin{array}{r}
a_{n} L_{n}^{(\alpha)}\left(\frac{u}{\beta}\right)=b_{n}\left(\frac{n !}{(\alpha+1)_{n}}\right)^{1 / 2} L_{n}^{(\alpha)}\left(\frac{u}{\beta}\right) \propto b_{n}\left(n^{-\alpha}\right)^{\frac{1}{2}} n^{\frac{\alpha}{2}-\frac{1}{4}}=b_{n} n^{-\frac{1}{4}} \\
\text { as } n \rightarrow+\infty, \text { for } u>0,
\end{array}
$$

where we also used $[5,6.1 .39]$ and $[7,8.22 .1]$. Again, $\alpha$ denotes proportionality with $n$ only; the exact scale factor will be presented in a later section where the errors of the approximations are estimated. So if $b_{n}$ decays faster than $n^{-3 / 4}$, the probability density function series in (91) converges absolutely.

For the generalized Laguerre series of the cumulative distribution function in (95), we have, for the envelope of the general term, 


$$
\begin{aligned}
& \frac{1}{n} a_{n} L_{n-1}^{(\alpha+1)}\left(\frac{u}{\beta}\right)=b_{n} \frac{1}{n}\left(\frac{n !}{(\alpha+1)_{n}}\right)^{1 / 2} L_{n-1}^{\left(\alpha^{+} 1\right)}\left(\frac{u}{\beta}\right)= \\
& \alpha b_{n} \frac{1}{n}\left(n^{-\alpha}\right)^{\frac{1}{2}}(n-1)^{\frac{\alpha^{+1}}{2}-\frac{1}{4}} \sim b_{n} n^{-3 / 4} \text { as } n \rightarrow+\infty, \quad \text { for } u>0 .
\end{aligned}
$$

Thus if $b_{n}$ decays faster than $n^{-1 / 4}$, (95) converges absolutely. And if the error integral (21) is finite, this property of the $\left\{b_{n}\right\}$ is true. So if error integral (21) is finite, the generalized Laguerre series for the cumulative distribution function converges absolutely for $u>0$; this is a sufficient, but not necessary, condition.

For zero argument, the generalized Laguerre polynomials behave differently for large n. From $[5,22.4 .7$ and 6.1.39],

$$
L_{n}^{(\alpha)}(0)=\left(\begin{array}{c}
n+\alpha \\
n
\end{array}\right)=\frac{(\alpha+1) n}{n !} \sim \frac{n^{\alpha}}{\Gamma\left(\alpha^{+} 1\right)} \text { as } n \rightarrow+\infty .
$$

Then (97) and (98) are both replaced by $b_{n} n^{\alpha / 2}$ as $n \rightarrow+\infty$. However, for $\alpha>0$, the probability density function in (91) is zero at $u=0$ due to the $u^{\alpha}$ term, so there is no need to perform the sum then. And the cumulative distribution function is always zero at $u=0$, again eliminating the need to evaluate the sum in (95). So the difference in behavior at $u=0$ is of no consequence. 
EXPANSION OF CHARACTERISTIC FUNCTION $f$

The coefficients $a_{n}$ and $c_{n}$ for the generalized Laguerre series were defined in (90) and (92). Then the sum

$$
\begin{aligned}
& \sum_{n=0}^{\infty} c_{n} w^{n}=\sum_{n=0}^{\infty} w^{n} \int_{0}^{\infty} d u p(u) L(\alpha)(u / \beta)= \\
& =\int_{0}^{\infty} d u p(u) \sum_{n=0}^{\infty} w^{n} L_{n}^{(\alpha)}(u / \beta)=\int_{0}^{\infty} d u p(u)(1-w)^{-\alpha-1} \exp \left(-\frac{u w / \beta}{1-w}\right)= \\
& =(1-w)^{-\alpha-1} f\left(\frac{-w / \beta}{1-w}\right),
\end{aligned}
$$

where $f$ is the characteristic function, and where we used $(90),[5,22.9 .15]$, and $(5)$. Thus $\left\{c_{n}\right\}$ are the expansion coefficients of the right-hand side of (100) in powers of $w$. If we let $w=\frac{-\beta z}{1-\beta z}$, we have the expansion for the characteristic function

$$
f(z)=(1-\beta z)^{-\alpha-1} \sum_{n=0}^{\infty} c_{n}\left(\frac{-\beta z}{1-\beta z}\right)^{n},
$$

corresponding to given probability density function $p$. Weighting parameters $\alpha$ and $B$ are arbitrary in (100) and (101).

Collecting (91) and (101) together for comparison, and assuming that $p_{N} \rightarrow p$ as $N \rightarrow+\infty$, we have, upon use of (92),

$$
\begin{aligned}
& p(u)=\frac{u^{\alpha} \exp (-u / \beta)}{\beta^{\alpha+1} \Gamma(\alpha+1)} \sum_{n=0}^{\infty} a_{n} \frac{(\alpha)}{n}\left(\frac{u}{\beta}\right) \text { for } u>0, \\
& f(i \xi)=(1-i \beta \xi)^{-\alpha-1} \sum_{n=0}^{\infty} a_{n} \frac{(\alpha+1) n}{n !}\left(\frac{-i \beta \xi}{1-i \beta \xi}\right)^{n} .
\end{aligned}
$$


TR 7377

Thus, expansion of probability density function $p$ in an infinite generalized Laguerre series is equivalent to an expansion of the corresponding characteristic function in the series of the particular form in (102). Equations (100)-(102) will serve as very convenient starting points for the derivation of several alternative recurrences for expansion coefficients $\left\{a_{n}\right\}$. We reiterate that $\alpha$ and $B$ are arbitrary in the above, except that $\alpha>-1, \beta>0$.

An analogous result holds for $N$ finite, but must be derived differently since we can no longer use infinite sum $[5,22.9 .15]$. Define the Fourier transform of (91) as the $\mathrm{N}$-th order approximation to the characteristic function:

$$
\begin{gathered}
f_{N}(i \xi)=\int d u \exp (i \xi u) p_{N}(u)= \\
=\int_{0}^{\infty} d u \exp (i \xi u) \frac{u^{\alpha} \exp (-u / \beta)}{\alpha^{\alpha+1} \Gamma\left(\alpha^{+}\right)} \sum_{n=0}^{N} a_{n} L_{n}^{(\alpha)}\left(\frac{u}{\beta}\right)= \\
=\frac{1}{\Gamma\left(\alpha^{+}-1\right)} \sum_{n=0}^{N} a_{n} \int_{0}^{\infty} d t \exp (i \delta \xi t) t^{\alpha} e^{-t} L_{n}^{(\alpha)}(t) .
\end{gathered}
$$

In appendix $D$, it is shown that

$$
\int_{0}^{\infty} d t e^{i \omega t} t^{\alpha} e^{-t} L_{n}^{(\alpha)}(t)=\frac{\Gamma\left(\alpha^{+} 1+n\right)}{n !} \frac{(-i \omega)^{n}}{(1-i \omega)^{\alpha^{+1+n}}} .
$$

Substitution in (103) then yields

$$
f_{N}(i \xi)=\sum_{n=0}^{N} a_{n} \frac{(\alpha+1) n}{n !} \frac{(-i \beta \xi)^{n}}{(1-i \beta \xi)^{\alpha^{+1+n}}}=\sum_{n=0}^{N} c_{n} \frac{(-i \beta \xi)^{n}}{(1-i \beta \xi)^{\alpha^{+1+n}}},
$$

where the last relation follows by use of (92). This result is the leading $N$ terms of (102). 
COEFFICIENTS RECURSIVELY VIA CUMULANTS

We can now obtain some useful recursive relations for expansion coefficients $\left\{a_{n}\right\}$ and/or $\left\{c_{n}\right\}$ in $(100)-(105)$. We start by taking the $h$ of $(100)$ :

$$
\ln \left\{\sum_{n=0}^{\infty} c_{n} w^{n}\right\}=-(\alpha+1) \ln (1-w)+\ln f\left(\frac{-w / \beta}{1-w}\right) .
$$

Identify the left-hand side as a new power series, and use (7) and $[5,15.1 .8]$ to yield

$$
\begin{aligned}
& \sum_{n=0}^{\infty} n_{n} w^{n}=(\alpha+1) \sum_{n=1}^{\infty} \frac{1}{n} w^{n}+\sum_{k=0}^{\infty} \frac{1}{k !} \chi_{k}\left(\frac{-w / \beta}{1-w}\right)^{k}= \\
& =(\alpha+1) \sum_{n=1}^{\infty} \frac{1}{n} w^{n}+\sum_{k=0}^{\infty} \frac{(-1)^{k} \chi_{k}}{k ! \beta^{k}} w^{k} \sum_{m=0}^{\infty} \frac{(k) m}{m !} w^{m} .
\end{aligned}
$$

Equating coefficients of $w^{n}$, there follows $h_{0}=\chi_{0}$, while for $n \geq 1$,

$$
\begin{aligned}
& h_{n}=\frac{1}{n}(\alpha+1)+\sum_{k=0}^{n} \frac{(-1)^{k} \chi_{k}}{k ! \beta^{k}} \frac{(k)_{n-k}}{(n-k) !}= \\
& =\frac{1}{n}\left[\alpha^{+1}+\sum_{k=1}^{n}(-1)^{k}\left(\begin{array}{l}
n \\
k
\end{array}\right) \hat{\chi}_{k}\right],
\end{aligned}
$$

where we used the normalized cumulants defined in (62).

But since the left-hand sides of (106) and (107) are equal, we have

$$
\sum_{n=0}^{\infty} c_{n} w^{n}=\exp \left\{\sum_{n=0}^{\infty} h_{n} w^{n}\right\} \text {, }
$$

or via appendix $A$, the recurrence 
TR 7377

$$
c_{n}=\frac{1}{n} \sum_{m=1}^{n} m h_{m} c_{n-m} \quad \text { for } n \geq 1, \quad c_{0}=\exp \left(h_{0}\right) .
$$

Finally, define

$$
d_{m}=m h_{m} \text { for } m \geq 1
$$

for notational convenience and thereby obtain

$$
\begin{aligned}
& d_{m}=\alpha^{+}+\sum_{k=1}^{m}(-1)^{k}\left(\begin{array}{l}
m \\
k
\end{array}\right) \hat{\chi}_{k} \text { for } m \geq 1, \\
& c_{n}=\frac{1}{n} \sum_{m=1}^{n} d_{m} c_{n-m} \text { for } n \geq 1, \quad c_{0}=\exp \left(x_{0}\right),
\end{aligned}
$$

by means of (108) and (110), respectively. Equation (112) is a recursive relation for expansion coefficients $\left\{c_{n}\right\}$ in terms of cumulants $\left\{x_{n}\right\}$ and auxiliary variables $\left\{d_{m}\right\}$. The $\left\{a_{n}\right\}$ are immediately available via (92).

As particular cases, we have, employing (62),

$$
\begin{aligned}
& c_{1}=\left(\alpha+1-\frac{\chi_{1}}{\beta}\right) c_{0}, \\
& c_{2}=\frac{1}{2}\left[(\alpha+2)(\alpha+1)-2(\alpha+2) \frac{\chi_{1}}{\beta}+\frac{\chi_{2}+\chi_{1}^{2}}{\beta^{2}}\right] c_{0} .
\end{aligned}
$$

Parameters $\alpha$ and $\beta$ are completely arbitrary in all the above equations, except that $\alpha>-1$ and $\beta>0$, and $\left\{X_{n}\right\}_{0}^{N}$ are the available cumulants.

Observe that if we

$$
\text { let } \begin{aligned}
\alpha^{+} 1 & =\frac{\chi_{1}^{2}}{\chi_{2}}=\frac{\mu_{1}^{2}}{\mu_{2} \mu_{0}-\mu_{1}^{2}}=\frac{M^{2}}{R^{2}} \\
\text { and } \quad \beta & =\frac{\chi_{2}}{\chi_{1}}=\frac{\mu_{2} \mu_{0}-\mu_{1}^{2}}{\mu_{1} \mu_{0}}=\frac{R^{2}}{M},
\end{aligned}
$$


then $c_{1}=0$ and $c_{2}=0$ (here we also used (8) and (25)); this is a special case of general property (33) stated earlier. Since the probability density function $\mathrm{p}(u)$ is nonzero only for $u>0$, then $\chi_{1}>0$ and $\chi_{2}>0$, giving allowable solutions to (114) in all cases. There then also follows, along with $d_{1}=d_{2}=0$ in this special case, the explicit results

$$
\begin{aligned}
& c_{0}=\exp \left(X_{0}\right), \quad c_{1}=0, \quad c_{2}=0, \\
& c_{3}=\frac{\chi_{1}^{2}}{3 ! \chi_{2}^{3}}\left(2 x_{2}^{2}-x_{3} x_{1}\right) c_{0} \text {, } \\
& c_{4}=\frac{\chi_{1}^{2}}{4 ! \chi_{2}^{4}}\left(18 x_{2}^{3}-12 x_{3} x_{2} x_{1}+\chi_{4} \chi_{1}^{2}\right) c_{0} \\
& c_{5}=\frac{\chi_{1}^{2}}{5 ! \chi_{2}^{5}}\left(144 \chi_{2}^{4}-120 x_{3} \chi_{2}^{2} x_{1}+20 \chi_{4} \chi_{2} \chi_{1}^{2}-\chi_{5} \chi_{1}^{3}\right) c_{0}, \\
& c_{6}=\frac{\chi_{1}^{2}}{6 ! \chi_{2}^{6}}\left(40\left(30 x_{2}+x_{1}^{2}\right)\left(x_{2}^{2}-x_{3} x_{1}\right) x_{2}^{2}+10 x_{3}^{2} x_{1}^{4}+\right. \\
& \left.+300 \chi_{4} \chi_{2}^{2} \chi_{1}^{2}-30 \chi_{5} \chi_{2} \chi_{1}^{3}+\chi_{6} \chi_{1}^{4}\right) c_{0}, \\
& c_{7}=\frac{\chi_{1}^{2}}{7 ! \chi_{2}^{7}}\left(10800 x_{2}^{6}+1260 x_{2}^{5} \chi_{1}^{2}-12600 \chi_{3} \chi_{2}^{4} \chi_{1}-1470 \chi_{3} \chi_{2}^{3} \chi_{1}^{3}+\right. \\
& +420 \chi_{3}^{2} \chi_{2} \chi_{1}^{4}+4200 \chi_{4} \chi_{2}^{3} \chi_{1}^{2}+70 \chi_{4} \chi_{2}^{2} \chi_{1}^{4}-35 \chi_{4} \chi_{3} \chi_{1}^{5}- \\
& \left.-630 x_{5} \chi_{2}^{2} \chi_{1}^{3}+42 \chi_{6} \chi_{2} \chi_{1}^{4}-\chi_{7} \chi_{1}^{5}\right) c_{0} \text {. }
\end{aligned}
$$


TR 7377

These relations have been confirmed by numerical comparison with (112).

These results greatiy extend those of $[1,(129)-(131)]$, where the equivalent of our $c_{3}$ is given (in terms of moments instead of cumulants, and with $\left.\chi_{0}=0\right)$, along with the comment that "the higher-order coefficients are so complicated that the whole value of this type of series seems to depend on the fact that the first term alone $\left(c_{0}\right)$ is often a good approximation." We find, on the other hand, that not only can we avoid the special choice in (114) and the corresponding complicated special results in (115), but we can handle any $\alpha, \beta$ pair and get very high-order coefficients $c_{n}$, simply by using the recurrence in (112), which is easily programmed. The only thing we lose are explicit results of the type given in (115); however, the latter are so complicated that they are of limited utility anyway.

\section{COEFF ICIENTS DIRECTLY VIA MOMENTS}

We will need the following expression $[5,22.3 .9]$ :

$$
\frac{n !}{(\alpha+1)_{n}} L_{n}^{(\alpha)}(x)=\frac{n !}{(\alpha+1)_{n}} \sum_{k=0}^{n}\left(\begin{array}{l}
n+\alpha \\
n-k
\end{array}\right) \frac{(-x)^{k}}{k !}=\sum_{k=0}^{n}\left(\begin{array}{l}
n \\
k
\end{array}\right) \frac{(-x)^{k}}{\left(\alpha^{+}+1\right)_{k}} .
$$

Then (92), (90), and (3) yield, for $n \geq 0$,

$$
\begin{aligned}
& a_{n}=\frac{n !}{\left(\alpha^{+} 1\right)_{n}} c_{n}=\frac{n !}{\left(\alpha^{+}+\right)_{n}} \int_{0}^{\infty} d u p(u) L_{n}^{(\alpha)}\left(\frac{u}{\beta}\right)= \\
& =\sum_{k=0}^{n}\left(\begin{array}{l}
n \\
k
\end{array}\right) \frac{1}{\left(\alpha^{+}+\right)_{k}} \int_{0}^{\infty} d u p(u)(-u / \beta)^{k}=\sum_{k=0}^{n}(-1)^{k}\left(\begin{array}{l}
n \\
k
\end{array}\right) \frac{\mu_{k}}{(\alpha+1)_{k} \beta^{k}} .
\end{aligned}
$$

It is useful, in this generalized Laguerre series case, to define an alternative set of normalized moments 


$$
\tilde{\mu}_{n}=\frac{\mu_{n}}{(\alpha+1)_{n} \beta^{n}} \text { for } n \geq 0
$$

(Although this seems to be very different from the earlier normalization in (69), (118) actually reduces to (69) for the a here equal to zero.) When (118) is utilized in (117), we have the desired expression for expansion coefficients $\left\{a_{n}\right\}$, directly in terms of (normalized) moments, in the surprisingly simple form

$$
a_{n}=\sum_{k=0}^{n}(-1)^{k}\left(\begin{array}{l}
n \\
k
\end{array}\right) \tilde{\mu}_{k} \text { for } n \geq 0 \text {. }
$$

Parameters $\alpha$ and $\beta$ in (118) are arbitrary, except that $\alpha>-1, \beta>0$.

As particular cases, (117)-(119) yield

$a_{0}=\mu_{0}, \quad a_{1}=\mu_{0}-\frac{\mu_{1}}{(\alpha+1) \beta}, \quad a_{2}=\mu_{0}-\frac{2 \mu_{1}}{(\alpha+1) \beta}+\frac{\mu_{2}}{(\alpha+1)(\alpha+2) \beta}$.

These agree with (113) which utilized cumulants. If we make the special choices of $\alpha^{+} 1=\mu_{1}^{2} /\left(\mu_{2} \mu_{0}-\mu_{1}^{2}\right)$ and $\beta=\left(\mu_{2} \mu_{0}-\mu_{1}^{2}\right) /\left(\mu_{1} \mu_{0}\right)$, then $a_{1}=0$ and $a_{2}=0$; this is a common approach to the approximation problem, but totaliy unnecessary.

An alternative derivation of the direct moment relation (117) is possible: from $(100),(6)$, and $[5,15.1 .8]$, 


$$
\begin{gathered}
\sum_{n=0}^{\infty} c_{n} w^{n}=(1-w)^{-\alpha-1} \sum_{k=0}^{\infty} \frac{1}{k !} \mu_{k}\left(\frac{-w / \beta}{1-w}\right)^{k}= \\
=\sum_{k=0}^{\infty} \frac{1}{k !} \mu_{k}\left(-\frac{w}{\beta}\right)^{k}(1-w)^{-\alpha-1-k}=\sum_{k=0}^{\infty} \frac{1}{k !} \mu_{k}\left(-\frac{w}{\beta}\right)^{k} \sum_{m=0}^{\infty} \frac{(\alpha+1+k)_{m} m}{m !} w^{m} .
\end{gathered}
$$

Equating coefficients of $w^{n}$, we have, for $n \geq 0$,

$c_{n}=\sum_{k=0}^{n} \frac{1}{k !} \mu_{k}\left(-\frac{1}{\beta}\right)^{k} \frac{(\alpha+1+k) n-k}{(n-k) !}=\frac{\left(\alpha^{+} 1\right)_{n}}{n !} \sum_{k=0}^{n}(-1)^{k}\left(\begin{array}{l}n \\ k\end{array}\right) \frac{\mu_{k}}{(\alpha+1)_{k} \beta^{k}}$,

which is equivalent to (117).

COEFF ICIENTS RECURSIVELY VIA MOMENTS

The starting point for this case is the characteristic function expansion in (101):

$f(z)=\sum_{m=0}^{\infty} c_{m}(-\beta z)^{m}(1-\beta z)^{-\alpha-1-m}=\sum_{m=0}^{\infty} c_{m}(-\beta z)^{m} \sum_{k=0}^{\infty} \frac{(\alpha+1+m) k}{k !}(\beta z)^{k}$

by use of $[5,15.1 .8]$. Now expand the left-hand side of (123) in powers of $z$, according to (6), and equate the coefficients of $z^{n}$ to get, for $n \geq 0$,

$\frac{1}{n !} \mu_{n}=\sum_{m=0}^{n} c_{m}(-\beta)^{m} \frac{\left(\alpha^{+} 1+m\right) n-m}{(n-m) !} \beta^{n-m}=\beta^{n} \sum_{m=0}^{n} \frac{c_{m}(-1)^{m}}{(n-m) !} \frac{\left(\alpha^{+1}\right)_{n}}{(\alpha+1)_{m}}$.

Therefore

$\frac{\mu_{n}}{\left(\alpha^{+}+1\right)_{n} \beta^{n}}=\sum_{m=0}^{n}(-1)^{m} \frac{n !}{(n-m) !} \frac{c_{m}}{(\alpha+1)}=\sum_{m=0}^{n}(-1)^{m}\left(\begin{array}{l}n \\ m\end{array}\right) a_{m}$ for $n \geq 0$, 
by use of (92). Then using normalized moment definition (118), (125) can be expressed as

$$
a_{n}=(-1)^{n}\left[\tilde{\mu}_{n}-\sum_{m=0}^{n-1}(-1)^{m}\left(\begin{array}{l}
n \\
m
\end{array}\right) a_{m}\right] \text { for } n \geq 0 \text {. }
$$

This is a recursive relation for expansion coefficients $\left\{a_{n}\right\}$ in terms of (normalized) moments. The parameters $\alpha$ and $\beta$ in (118) are arbitrary, except that $\alpha>-1, \beta>0$.

SUMMARY

The approximations to the probability density function and cumulative distribution function are given by (91) and (95), respectively, where $\alpha$ and $\beta$ are arbitrary constants, except that $\alpha>-1, \beta>0$. The generalized Laguerre polynomials are available via (96). The expansion coefficients $\left\{a_{n}\right\}$ are given by the three alternatives (112), (119), (126), in terms of normalized cumulants (62) and normalized moments (118); in the case of (112), the interrelationship between expansion coefficients $\left\{a_{n}\right\}$ and $\left\{c_{n}\right\}$ is given in (92). Programs for all three alternative procedures for determining the expansion coefficients $\left\{a_{n}\right\}$ are listed in an appendix. The basis for these relations is the characteristic function expansion in (100)-(102). 
TR 7377

\section{EXAMPLES OF HERMITE EXPANSION}

\section{EXAMPLE A}

The first example is one which can be handled analytically, and thereby furnishes checks on numerical procedures and results. Consider the Gaussian probability density function

$$
p(u)=\frac{1}{\omega} \phi\left(\frac{u-\gamma}{\omega}\right) \quad(\omega>0)
$$

with cumulative distribution function and characteristic function

$$
P(u)=\Phi\left(\frac{u-\gamma}{\omega}\right), f(i \xi)=\exp \left(i \xi \gamma-\frac{1}{2} \xi^{2} \omega^{2}\right) .
$$

The cumulants are

$$
\chi_{0}=0, \quad \chi_{1}=r, \quad \chi_{2}=\omega^{2}, \quad \chi_{n}=0 \text { for } n \geq 3 \text {, }
$$

while the moments are most easily evaluated by the recurrence

$$
\mu_{n}=\gamma \mu_{n-1}+(n-1) \omega^{2} \mu_{n-2} \text { for } n \geq 2, \mu_{0}=1, \mu_{1}=r .
$$

It is obvious in this Hermite expansion case that the best choice of weighting parameters would be $\alpha=\gamma, \beta=\omega$, for then weighting $w$ would match $p$ perfectly and there would follow $b_{n}=0$ for $n \geq 1$. We consider a mismatched choice of $\alpha$ and $\beta$ to illustrate rapid decay of the expansion coefficients and some conditions on convergence.

\footnotetext{
Expansion coefficient $C_{n}$ follows from (45) and (127A) according to
} 


$$
\begin{aligned}
& c_{n}=\int d u p(u) H e_{n}\left(\frac{u-\alpha}{\beta}\right)=\beta \int d x p(\alpha+\beta x) H e_{n}(x)= \\
& =\beta \int d x \frac{1}{\omega} \phi\left(\frac{\alpha-\gamma+\beta x}{\omega}\right) H e_{n}(x)=\left(\frac{\sqrt{\beta^{2}-\omega^{2}}}{\beta}\right)^{n} H e_{n}\left(\frac{\gamma-\alpha}{\sqrt{\beta^{2}-\omega^{2}}}\right),
\end{aligned}
$$

the last step via use of $[5,22.5 .18]$ and $[8,7.37410]$. Then from (47),

$$
b_{n}=(n !)^{-1 / 2}\left(\frac{\sqrt{\beta^{2}-\omega^{2}}}{\beta}\right)^{n} \quad H_{n}\left(\frac{\gamma-\alpha}{\sqrt{\beta^{2}-\omega^{2}}}\right) \text { for } n \geq 0 \text {. }
$$

This equation is correct for all positive values of $\beta$ and $\omega$. However, for $\beta<\omega$, a more convenient form can be obtained by use of (74), if desired:

$$
b_{n}=(n !)^{-1 / 2}\left(\frac{\sqrt{\omega^{2}-\beta^{2}}}{\beta}\right)^{n} H i n\left(\frac{\gamma-\alpha}{\sqrt{\omega^{2}-\beta^{2}}}\right)
$$

where $\mathrm{Hi}_{\mathrm{n}}$ is the modified Hermite polynomial. For $\beta=\omega$, a limit of (130) yields $b_{n}=(n !)^{-1 / 2}((\gamma-\alpha) / B)^{n}$.

If $B>\omega$, we can use the result in (50A) on (130) and obtain

$$
b_{n} \propto\left(\frac{\sqrt{\beta^{2}-\omega^{2}}}{\beta}\right)^{n} n^{-1 / 4} \text { as } n \rightarrow+\infty \text {. }
$$

Since the quantity in parentheses is always less than 1 in this case of $\beta>\omega$, we have $b_{n} \rightarrow 0$ as $n \rightarrow+\infty$.

For $\beta<\omega$, we use [7, theorem 8.22.7] and find now that

$$
b_{n} \propto \exp (\sqrt{2 n} A)\left(\frac{\sqrt{\omega^{2}-\beta^{2}}}{\beta}\right)^{n} n^{-1 / 4} \text { as } n \rightarrow+\infty
$$


TR 7377

where $A$ is the absolute value of the argument of $\mathrm{He}_{n}$ in (130). This quantity (133) tends to zero with $n$, regardless of $A$, when $\beta>\omega / \sqrt{2}$.

Combining with the result above, we can conclude that

$$
b_{n} \rightarrow 0 \text { as } n \rightarrow+\infty \quad \text { for } \frac{\omega}{\sqrt{2}}<\beta<+\infty
$$

Furthermore, $b_{n}$ behaves as an $n$-th power, which is faster than $n^{-1 / 4}$, thereby guaranteeing convergence of the probability density function and cumulative distribution function series, according to the discussion in (50A) et seq. On the other hand, $\left\{b_{n}\right\}$ diverges when $0<\beta<\omega / \sqrt{2}$, as may be seen from (133).

The error integral in (21) is, for Hermite weighting (40) and probability density function (127),

$$
\int d u \frac{p^{2}(u)}{w(u)}=\frac{\beta^{2}}{\omega \sqrt{2 \beta^{2}-\omega^{2}}} \exp \left(\frac{(\gamma-\alpha)^{2}}{2 \beta^{2}-\omega^{2}}\right) \text { if } \frac{\omega}{\sqrt{2}}<\beta,
$$

by use of $[8,3.3232]$; this integral is divergent if $\beta<\omega / \sqrt{2}$. Thus, for this particular example, the error integral and expansion coefficient sequence $\left\{b_{n}\right\}$ converge or diverge together, depending on the condition $\beta \gtrless \omega / \sqrt{2}$. The choice of $\alpha$ is irrelevant in this case.

A numerical example of sequence $\left\{b_{n}\right\}$ for

$$
\gamma=1.1, \omega=2.3 \quad \alpha=1.14, \beta=2.34
$$


is plotted in figure 1 on a logarithmic ordinate. Values of $b_{n}$ less than $1 E-7$ in absolute value are all plotted at the $\pm 1 E-7$ line. The critical ratio $\sqrt{\beta^{2}-\omega^{2}} / \beta$ in (130) is .184 for this example, leading to rapid decay of expansion coefficients $\left\{b_{n}\right\}$. The three sets of expansion coefficients in figure 1 are labelled according to the shorthand notation

$$
\begin{aligned}
& \text { RC: Recursively via Cumulants, } \\
& \text { DM: Directly via Moments, } \\
& \text { RM: Recursively via Moments. }
\end{aligned}
$$

It is seen that the expansion coefficients determined recursively via cumulants, namely, the RC plot, decay rapidly and never encounter round-off error, whereas the DM and RM procedures both are subject to large round-off error for $n>70$, as indicated by the large increasing oscillations. This example can be rather misleading, however, since all the cumulants (128A) of Gaussian probability density function (127A) are zero, except for $\chi_{1}=r, \chi_{2}=\omega^{2}$; this leads to a very special form of the RC procedure unique to the Gaussian case.

In figure 2, the cumulative distribution function and exceedance distribution function, 1-P(u), as determined by Hermite expansion (48) using $N=50$ terms, are plotted. The exact result, (1278), overlapped these curves over the full range plotted. The three procedures, RC, DM, and RM, all yielded identical distributions in figure 2, as inspection of figure 1 confirms, since the three sets of expansion coefficients are virtually the same for $n<50$. Even though the three sets of expansion coefficients differ significantly for $n>60$, the corresponding approximate probability density 


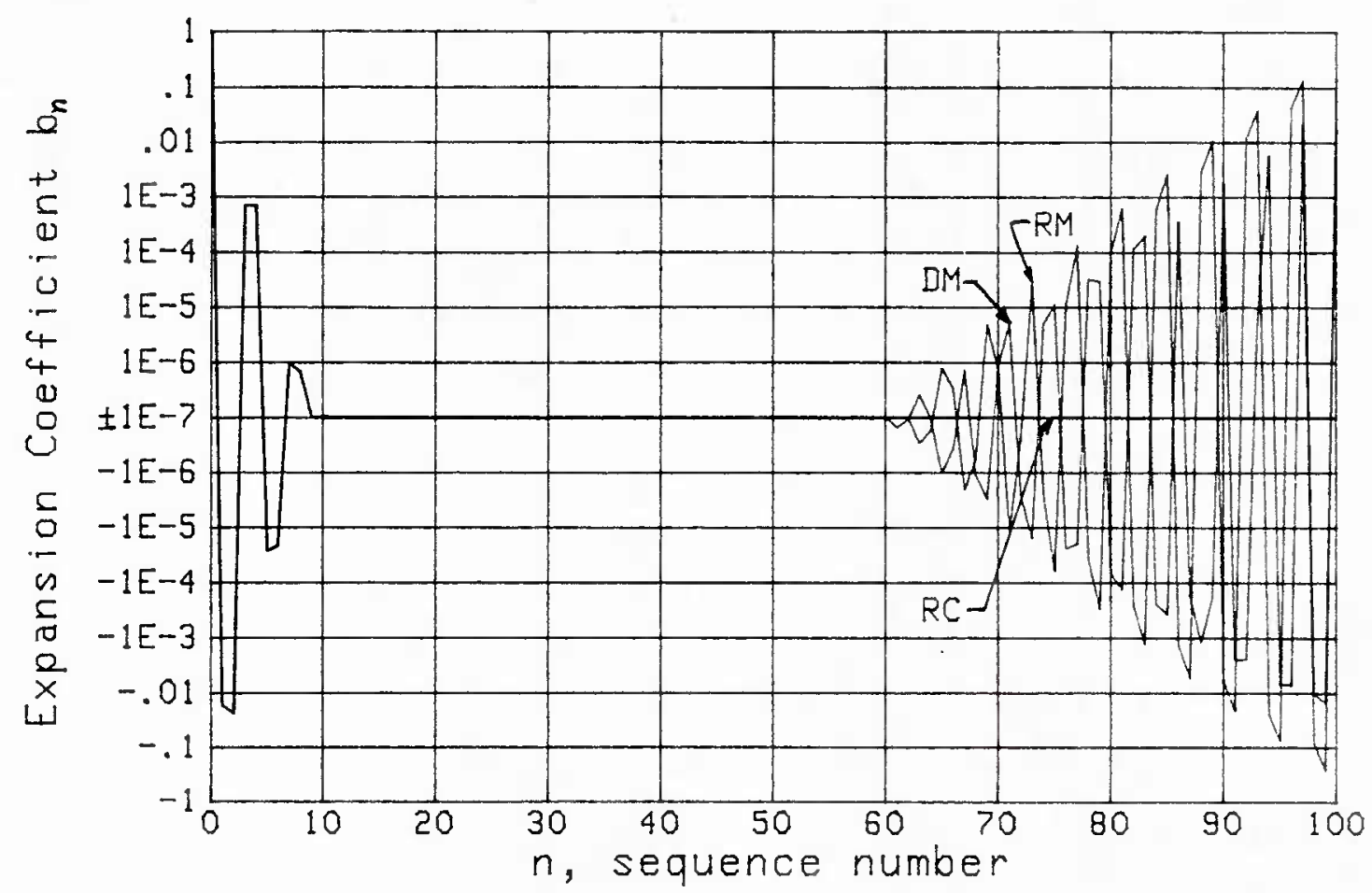

Figure 1. Hermite Coefficients for Example $A$

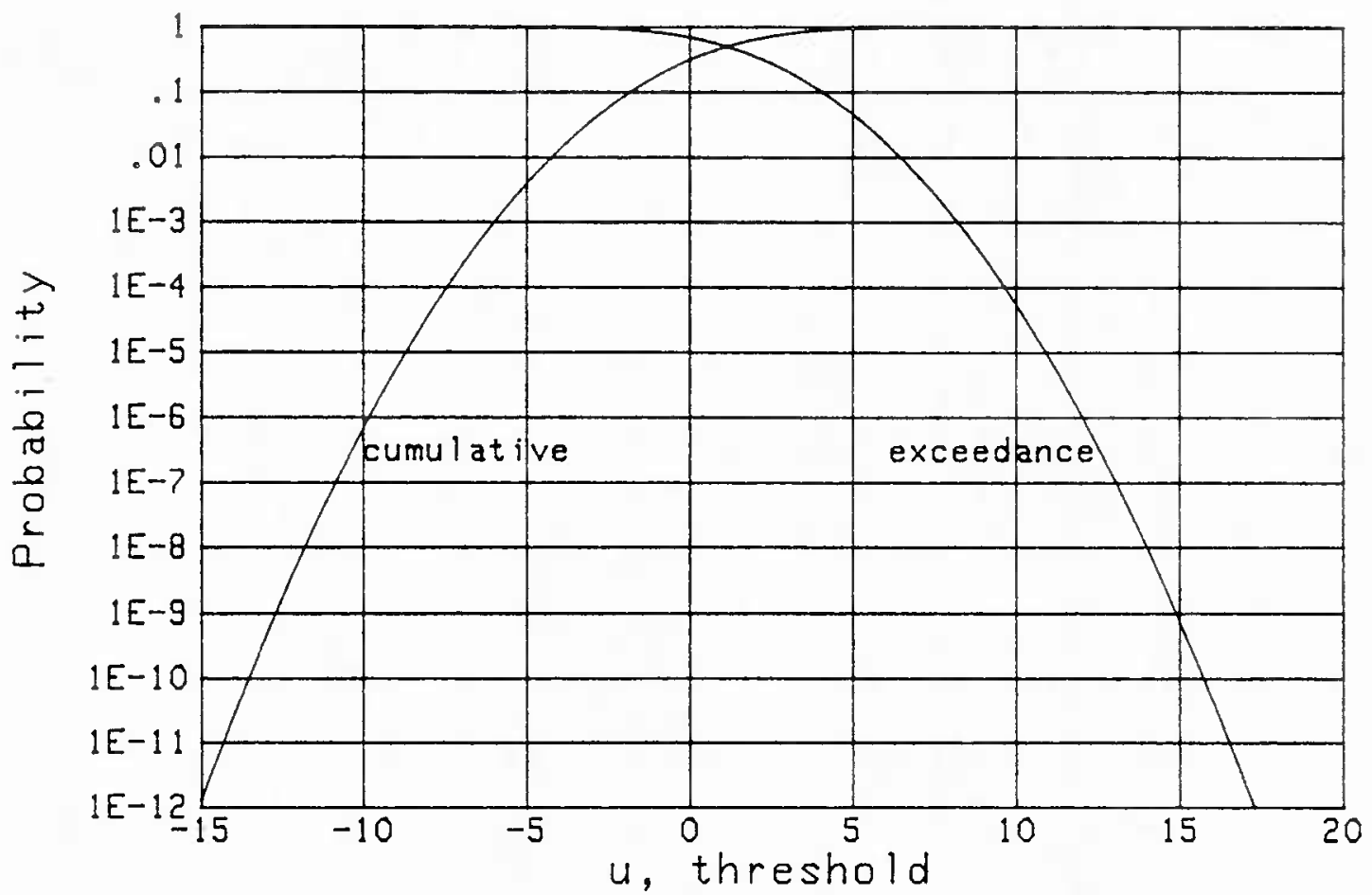

Figure 2. Distributions for Example $A$ 
TR 7377

functions and cumulative distribution functions for $N=70$, say, would not be very different, because the relative differences in $p$ and $P$ are very small, somewhere, in the $1 E-5$ range; see figure 1 for $n=70$, and recal1 that $b_{0}=1$ for this example.

EXAMPLE B

The probability density function of interest here is the one previously considered in (34) et seq.:

$$
p(u)=\frac{2 u^{\gamma} \exp \left(-u^{2} / \omega^{2}\right)}{\omega^{\gamma^{+}} \Gamma\left(\frac{\gamma^{+}}{2}\right)} \text { for } u>0 \quad(\gamma>-1, \omega>0) \text {. }
$$

This class of probability density functions includes, for $\gamma=0,1,2$, respectively, the one-sided Gaussian, Rayleigh, and Maxwell as special cases. The characteristic function and cumulants are not easily determined directiy for this function. However, the moments, as given already in (35), are readily evaluated via the simple recursion

$$
\mu_{n}=\mu_{n-2} \frac{\omega^{2}}{2}(\gamma-1+n) \quad \text { for } n \geq 2, \quad \mu_{0}=1, \quad \mu_{1}=\omega \frac{\Gamma\left(\frac{\gamma}{2}+1\right)}{\Gamma\left(\frac{\gamma^{+}}{2}\right)} \text {. }
$$

An example of the expansion coefficients for

$$
\gamma=3, \omega=1 \quad \alpha=0, \beta=.7
$$

is depicted in figure 3 . The values of $b_{n}$ for $n=0,1,2,3$ are 1, 1.90, $2.18,1.63$, respectively, and lie above the top of the plotted region. The 


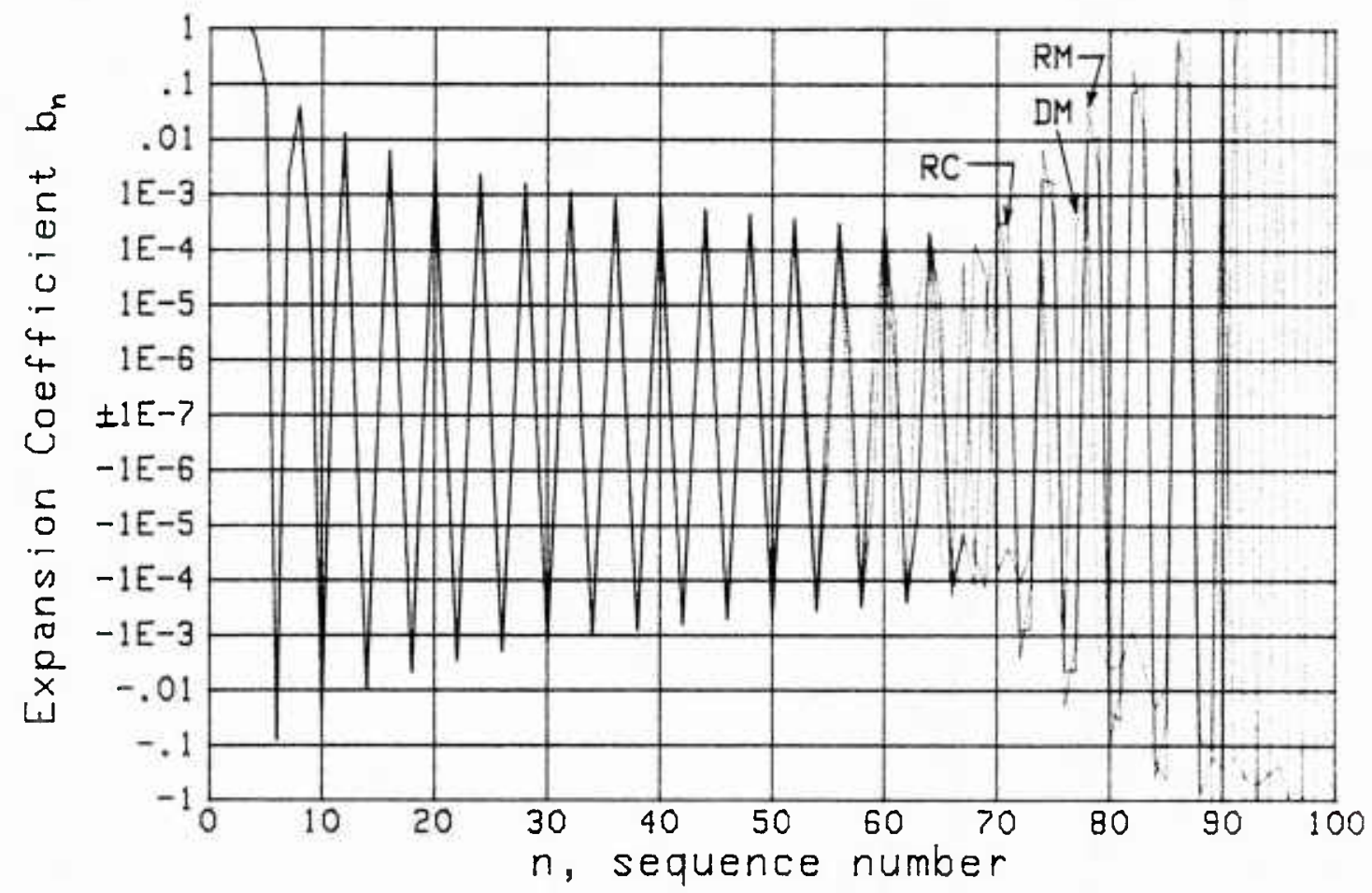

Figure 3. Hermite Coefficients for Example B

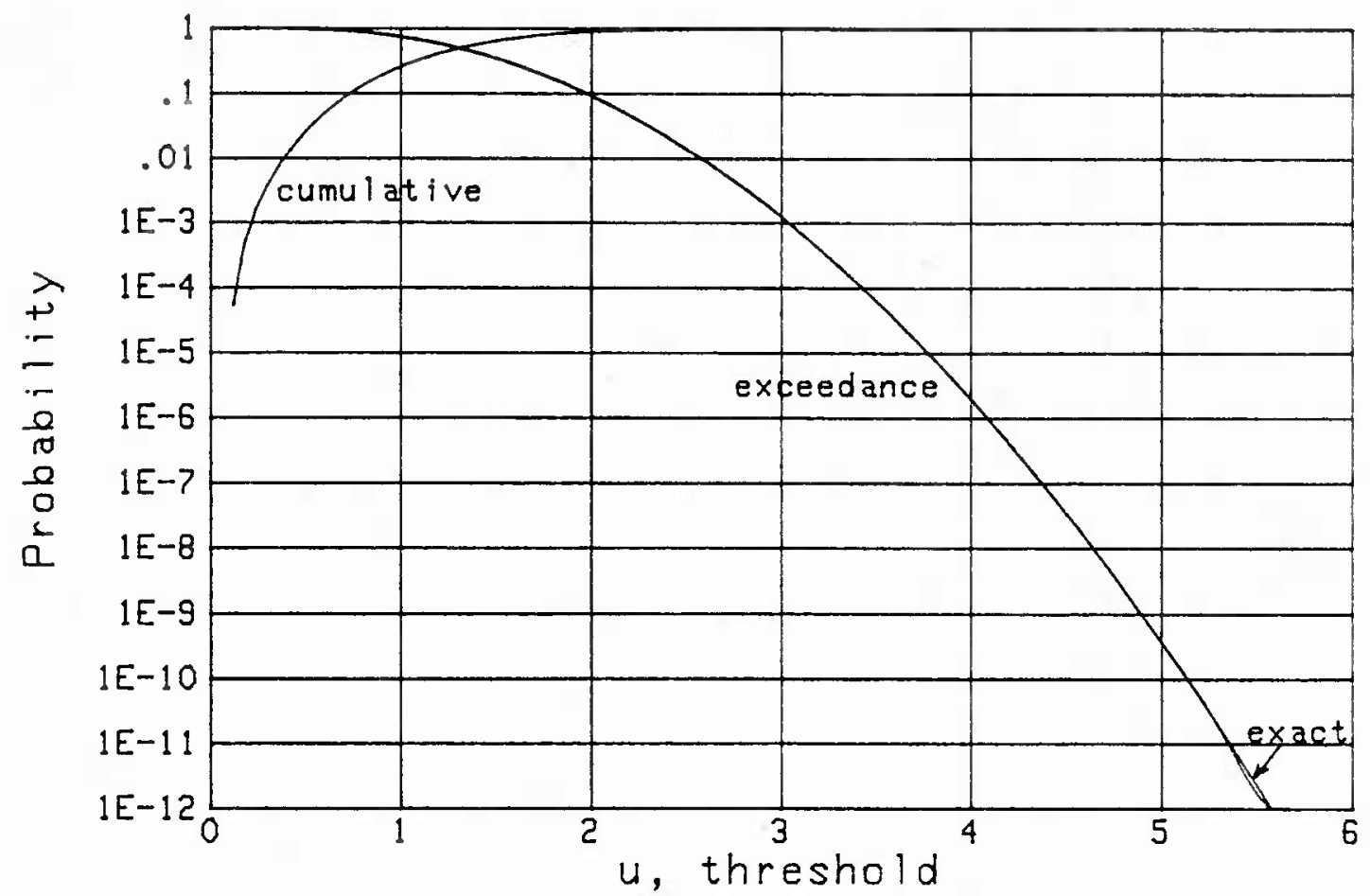

Figure 4. Distributions for Example B 
TR 7377

coefficients obtained directly via moments, DM, decay to approximately $1 E-4$ near $n=70$ and then encounter round-off error. The expansion coefficients corresponding to $R C$ and $R M$ are more noisy. The procedure used for $R C$ was to determine the moments via (139), transform directly to cumulants according to $(A-7)$, and then use (63).

A plot of the distributions using $N=65$ terms is given in figure 4 ; the results are the same for all three sets of expansion coefficients, as may be seen by reference to figure 3. Furthermore, the exact cumulative distribution function, $P(u)=1-\left(1+u^{2}\right) \exp \left(-u^{2}\right)$ for $u>0$, overlays these results except for the bow in the exceedance distribution function below $1 E-11$ near $u=5.5$. Values of the cumulative distribution function for $u<0$ as determined by series (48) are not zero, although they should be for this example; the generalized Laguerre series would fit this example better, since it is nonzero only for positive arguments.

EXAMPLE C

Consider the class of Bessel-function probability density functions

$$
p(u)=\Lambda u^{\gamma} \exp \left(-u^{2} / \omega^{2}\right) I_{\rho}(\theta u) \text { for } u>0 \text {, }
$$

which includes the Rice and generalized $Q_{M}$ distributions, for example. The $n$-th moment is $[8,6.6311]$

$$
\mu_{n}=\Lambda \frac{\theta^{\rho} \Gamma\left(\frac{n}{2}+h\right) \omega^{n+2 h}}{2^{\zeta+1} \Gamma(\rho+1)} 1 F_{1}\left(\frac{n}{2}+h ; \rho+1 ; \frac{1}{4} \omega^{2} \theta^{2}\right) \text { for } n \geq 0 \text {, }
$$


TR 7377

with $h=(\gamma+\zeta+1) / 2$; in order for $\mu_{0}$ to be finite, we must have $h>0$. The ${ }_{1} F_{1}$ function in (142) can be evaluated via recursion; this leads to a recursion for the moments (see appendix $E$ ).

We consider here only the special case of the Rice probability density function, namely,

$$
\Lambda=\frac{2}{\omega^{2}} \exp \left(-\frac{1}{4} \omega^{2} \theta^{2}\right), \quad \gamma=1, \quad J=0,
$$

for which

$$
p(u)=\frac{2 u}{\omega^{2}} \exp \left(-\frac{u^{2}}{\omega^{2}}-\frac{\omega^{2} \theta^{2}}{4}\right) I_{0}(\theta u) \text { for } u>0 \text {. }
$$

The moments in (142) then reduce to

$$
\mu_{n}=\Gamma\left(\frac{n}{2}+1\right) \omega^{n}{ }_{1} F_{1}\left(-\frac{n}{2} ; 1 ;-\frac{1}{4} \omega^{2} \theta^{2}\right),
$$

and can be easily determined by the recurrence presented in $(E-5)$. The cumulative distribution function corresponding to (144) is the Q function [1]

$$
P(u)=1-Q\left(\frac{\omega \theta}{\sqrt{2}}, \frac{\sqrt{2} u}{\omega}\right) \text { for } u>0 \text {; }
$$

the characteristic function is given in [9, appendix A] as an infinite series, meaning that the cumulants cannot be determined directly, except via the moments.

The particular example we consider here for the Hermite expansion is a sum of 8 independent random variables, each with Rice probability density function (144). For direct comparison with the exact results in [9], we also consider the normalized form of (144), namely $\omega^{2}=2$. Furthermore, we limit 
numerical consideration in this particular example to evaluation of the cumulative and exceedance distribution functions for $\theta=0$, which corresponds physically to the false alarm probability for the sum of eight normalized envelopes of narrowband Gaussian noise (i.e., a Rayleigh probability density function for the individual random variables).

For $\alpha=4, \beta=2.15$, the expansion coefficients $\left\{b_{n}\right\}$ are displayed in figure 5 for the RC, DM, and $P M$ approaches. All the $\left\{b_{n}\right\}$ for $1 \leq n \leq 20$ are bigger than 1 ; the biggest is $b_{6}=12.25$. The $\left\{b_{n}\right\}$, for both moment approaches, have not been plotted for $n>60$ because they continue to oscillate well beyond the \pm 1 limits, while the RC coefficients decay exponentially with $n$. Despite the fact that the moments were the initially determined quantities for this example, the RC method far outperforms the DM and RM methods, as seen in figure 5. The reason for this is as follows: for the RC method, the procedure was to obtain moments via (145), cumulants via $(A-7)$, cumulants of the sum of 8 independent random variables by simple scaling by a factor of 8 , and then expansion coefficients via (63). For the DM and RM methods, the moments of the sum of 8 random variables were determined via $[6,(14)]$ which progressively determined the moments of a sum of 2 random variables, then $3,4, \ldots, 8$ in order, and then employed (70). This iterated procedure for moments requires more number-crunching and leads to considerably larger round-off error than the simple scaling required for the RC procedure. Thus it appears that when the random variable of interest is obtained as a sum of several independent random variables, the RC approach will be the prime candidate for expansion coefficient evaluation; this applies also if the individual random variables have different statistics, but remain independent. 


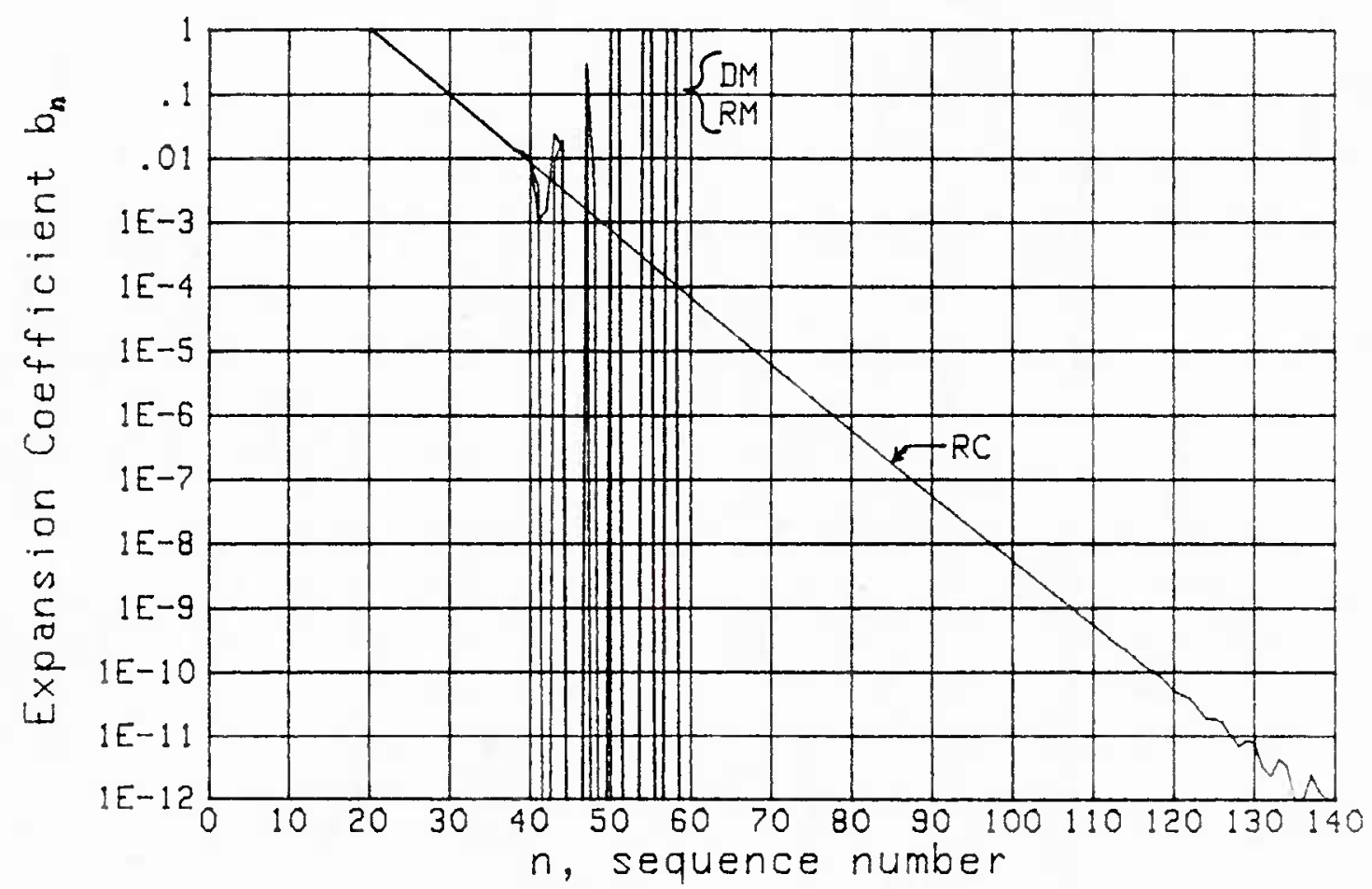

Figure 5. Hermite Coefficients for Example $C$

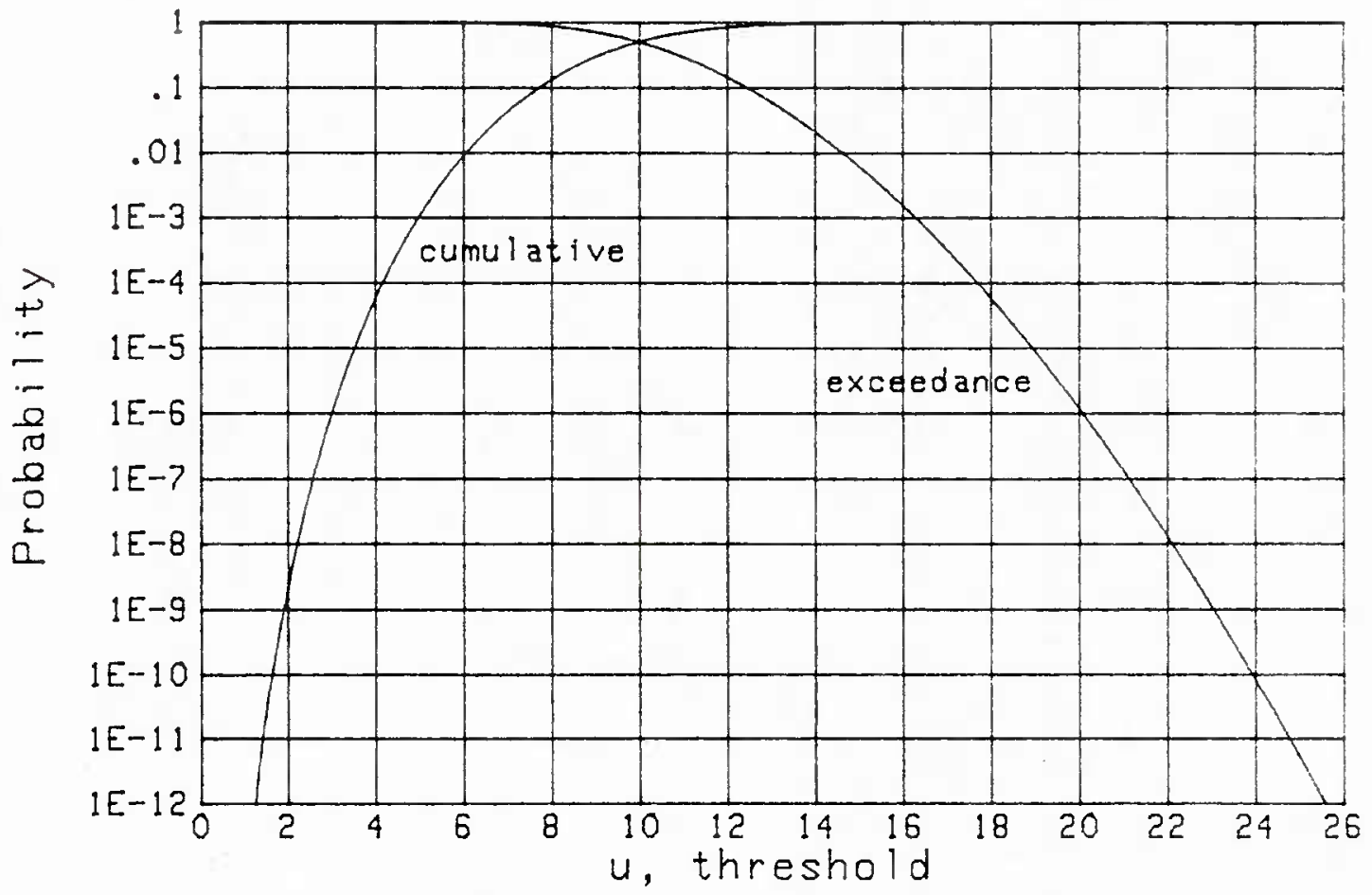

Figure 6. Distributions for Example C 
The cumulative and exceedance distribution functions for this sum of 8 normalized Rayleigh variates are plotted in figure 6 , for the $N=140$ expansion coefficients of the $R C$ procedure in figure 5 . In order to make a precise determination of the accuracy of this Hermite series approach, the false alarm probabilities were computed at the eight thresholds listed under $M=8$ in [9, table 1]. To the precision given in that table, the computed probabilities were exactly the specified values $1 E-m$ for $m=1(1) 8$. Thus, as anticipated by figure 5, very accurate evaluation of false alarm probabilities are possible by this series approach.

A short search of values of the best weighting parameters $\alpha$ and $\beta$, to use with the DM approach, led to $\alpha=5.84 ; \beta=2.28$ and expansion coefficients $b_{n}$ near $1 E-4$ at $n=28$, before round-off error became dominant. This is better than the result of DM in figure 5 for $\alpha=4, \beta=2.15$. Evaluation of the false alarm probabilities at the thresholds in [9, table 1] gave 7 decimal accuracy at .1, and 4 decimal accuracy at $1 E-8$. This is adequate for most purposes, but is not as good as the RC approach.

\section{EXAMPLE D}

In [4, appendix C], the characteristic function for shot noise with random amplitude and duration modulation, and arbitrary individual pulse shape, is derived. (This result is then specialized to elliptical pulses and Rayleigh amplitude modulation $[4,(C-36)-(C-42)]$.$) Also, the cumulants are$ extracted, with general result $[4,(24)]$, where $v$ is the average number of pulses/second, $\bar{l}$ is the average length of the duration modulation, $\mu_{a}(n)$ is 
TR 7377

the $n$-th moment of the amplitude modulation, and $F(x)$ is the individual pulse shape of the shot noise. Thus shot noise is a case where the cumulants are directly capable of evaluation, whereas the moments must be found indirectly.

For the special case of elliptical pulses and Rayleigh amplitude modulation, there follows for the cumulants $[4,(29)]$ :

$$
\chi_{n}=v \bar{l} \sigma_{a}^{n} 2^{\frac{3}{2} n+1} \Gamma^{3}\left(\frac{n}{2}+1\right) / \Gamma(n+2) \quad \text { for } n \geq 1, \quad \chi_{0}=0 .
$$

These quantities are easily evaluated via recurrence

$$
\chi_{n}=\chi_{n-2} \sigma_{a}^{2} n^{2} /(n+1) \text { for } n \geq 3, \chi_{1}=\left(\frac{\pi}{2}\right)^{3 / 2} v \bar{l} \sigma_{a}, \chi_{2}=\frac{8}{3} v \bar{l} \sigma_{a}^{2} \text {. }
$$

This procedure was used in [4, appendix 0] to obtain the probability density function and cumulative distribution function results given there.

There is a nuance that arises in shot noise for pulse shapes of finite duration; see [4, pp. 40-42]. Namely, there is an impulse in the probability density function, at $u=0$, of area

$$
P_{0}=\exp \left[-\nu \bar{l}\left(x_{2}-x_{1}\right)\right] \text {, }
$$

where $\left(x_{1}, x_{2}\right)$ is the non-zero extent of an unmodulated individual pulse. Since an impulse is very difficult to approximate by a finite series of continuous functions, the effect of this quantity should be subtracted from the statistics (moments or cumulants), and the continuous portion of the probability density function should be approximated. Similarly, the 
corresponding step in the cumulative distribution function at the origin should be eliminated from the approximation procedure.

This feature is easily incorporated if $P_{0}$ is subtracted from the zero-th order moment [4, p. 42]. The only undesireable side-effect of this manipulation is that the initially computed cumulants must be transformed to moments, then $\mu_{0}$ corrected, and then all the new cumulants evaluated. This double transformation is necessary because the correction (subtraction) procedure can onty be accomplished in the moment domain. Of course, when the DM or RM procedures are employed instead of RC, the last transformation to cumulants is unnecessary; this was, in fact, the procedure used in [4, p. 60].

When the individual pulse $F(x)$ has infinite duration, as for an exponential or Gaussian waveform, then $x_{2}-x_{1}$ is infinite and $P_{0}$ in (149) is zero. In that case, the considerations in the last two paragraphs can be disregarded, and the cumulants generated via (148) used as is. It is then very likely that even better accuracy in the expansion coefficients will be achieved than for this current example.

$$
\begin{aligned}
& \text { For overlap factor }[4, p .43] \\
& \qquad \bar{K}_{1}=v \bar{\ell}\left(x_{2}-x_{1}\right)=6.2, \quad P_{0}=\exp (-6.2)=.00203, \quad \sigma_{a}=1,
\end{aligned}
$$

and for weighting parameters $\alpha=6.1, \beta=4.3$, the expansion coefficients $\left\{b_{n}\right\}$ are displayed in figure 7 for the three recursive procedures. The RM results are considerably poorer than the $\mathrm{RC}$ and $\mathrm{DM}$ coefficients, which are 


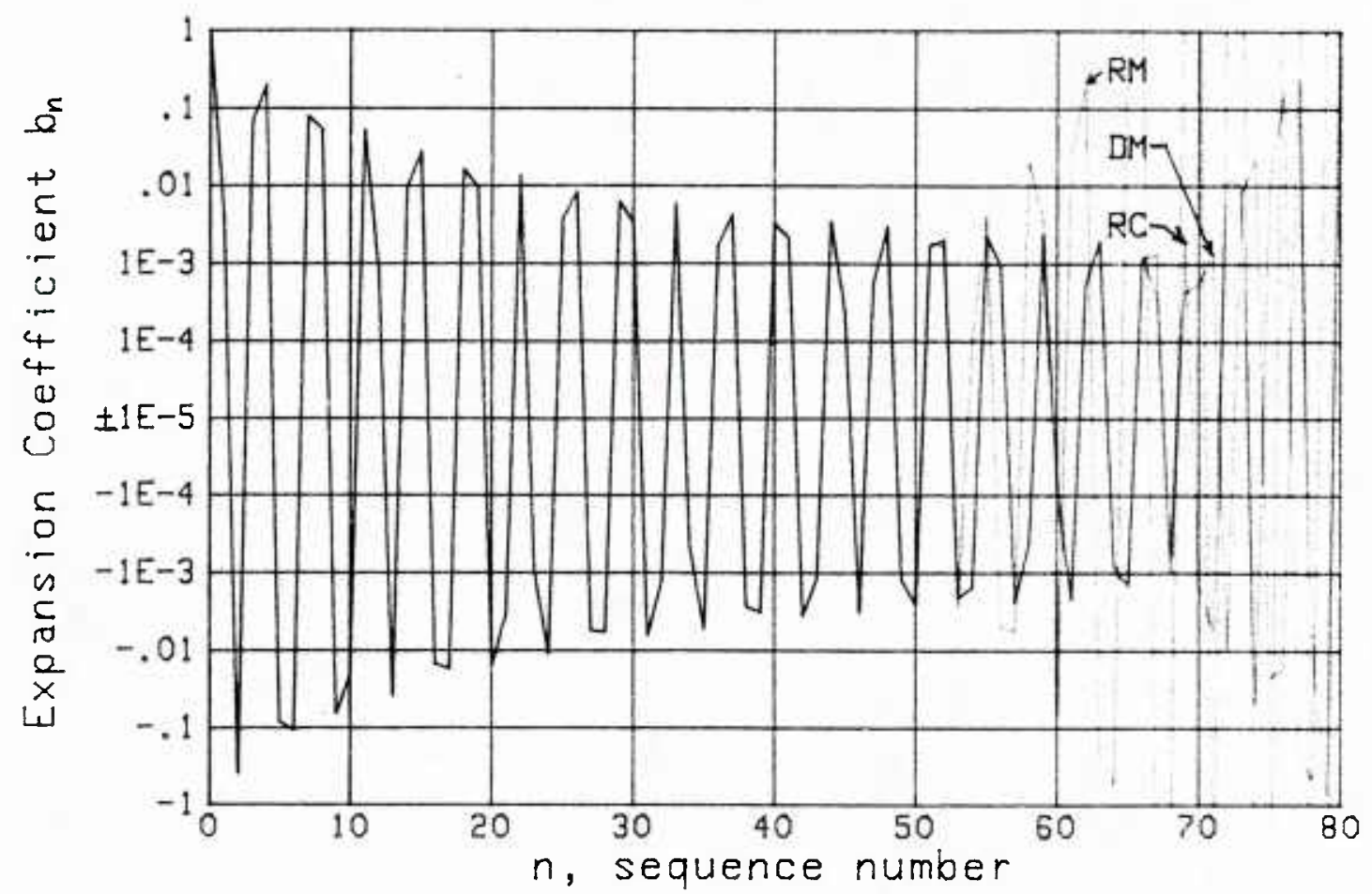

Figure 7. Hermite Coefficients for Example $D$

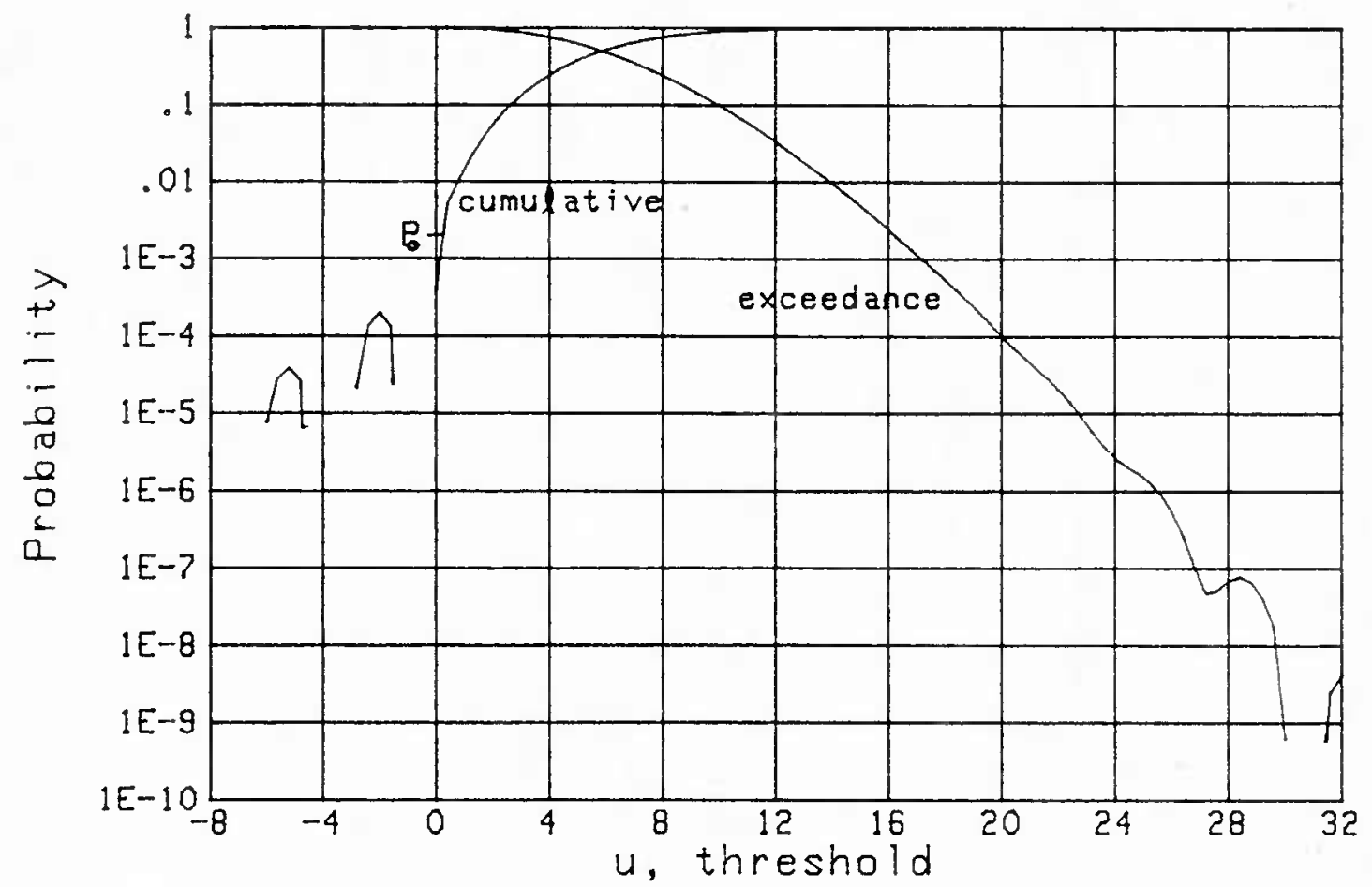

Figure 8. Distributions for Example D 
comparable for $n<65$. However, even here, the coefficients have only decayed to the 1E-3 level, which may not be sufficiently small for accurate results.

The distributions using $N=65$ terms and the $R C$ expansion coefficients are given in figure 8. Although the actual cumulative distribution function is zero for $u<0$, the approximation oscillated around zero, reaching a positive peak of value .22E-3 at $u=-2$. Similarly, significant wiggles develop in the exceedance distribution function below the $1 E-4$ level. The reason for the inadequacy of these Hermite expansions near $u=0$ is the abrupt zero behavior of the true probability density function for negative arguments, a feature inherently difficult to approximate by means of smooth continuous functions. The error of the approximations in figure 8 is estimated in a later section and superposed on the plot, for ease of ascertaining the reliability of the curves. The corresponding approximations for the generalized Laguerre series are better for this type of probability density function, as will be demonstrated in the next section.

The approximate probability density function for this example, again with $N=65$ terms, is given in figure 9 on a linear ordinate. It reaches a negative peak of $-8 E-4$, and crosses the $u=0$ axis with value .004 ; both of these values should be zero, and will be for the generalized Laguerre series. To see how the approximate probability density function behaves for larger arguments, the logarithmic plot in figure 10 is used. Wiggles develop near the $1 \mathrm{E}-4$ level and become large enough that negative values of the density are yielded near $u=28$ and 31 . It will be worthwhile to compare this Hermite series with the generalized Laguerre series to be presented in the next section. The estimated error associated with figure 10 is developed in a later section. 


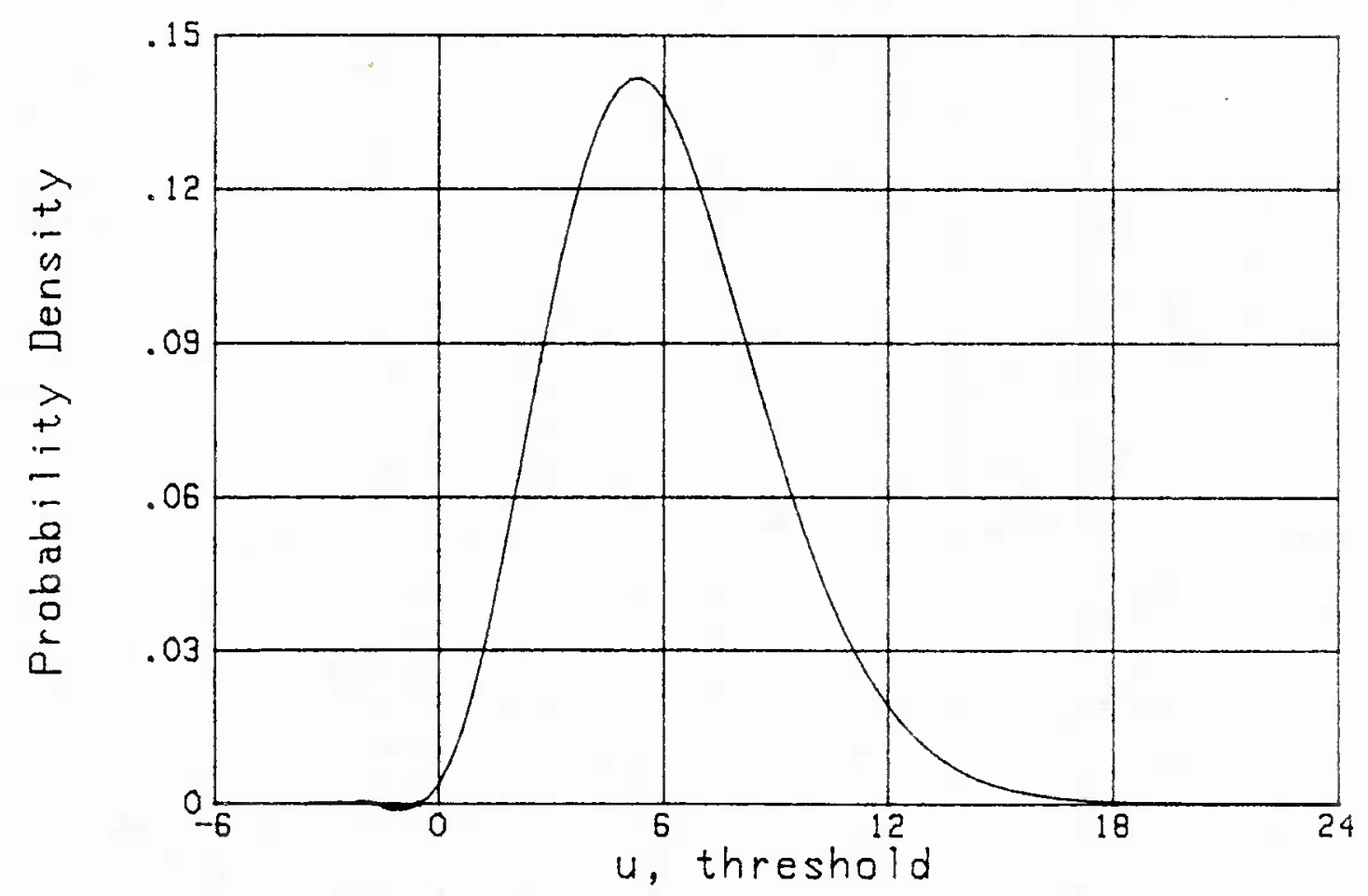

Figure 9. Linear Density for Example $D$

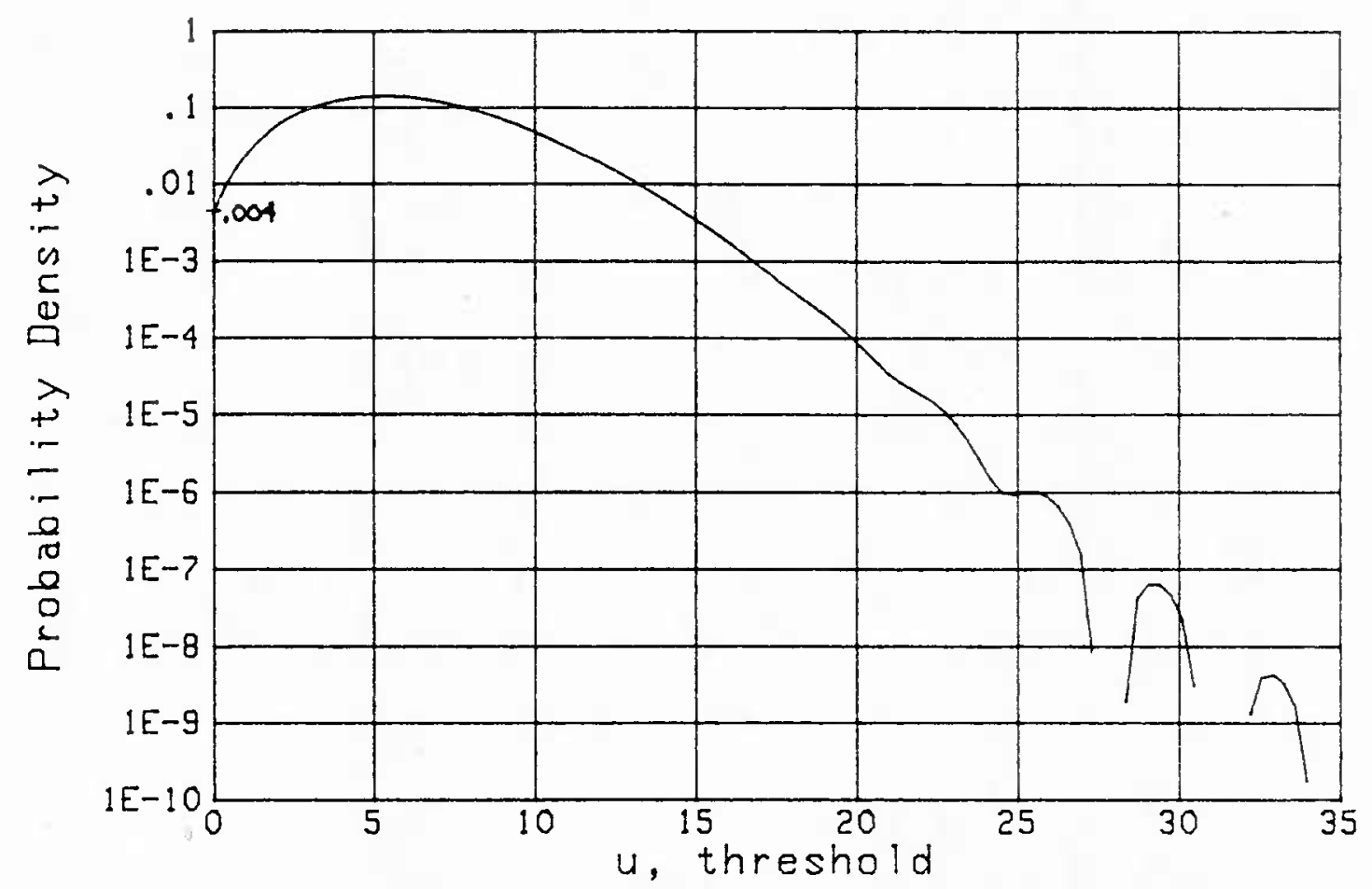

Figure 10. Log Density for Example D 


\section{EXAMPLES OF GENERAL IZED LAGUERRE EXPANS ION}

\section{EXAMPLE E}

As with the earlier Hermite expansions, the first generalized Laguerre example here is one that can be evaluated analytically, for purposes of checking numerical procedures and results. Namely consider the Chi-square probability density function of $2\left(\gamma^{+} 1\right)$ degrees of freedom (which need not be integer):

$$
p(u)=\frac{u^{\gamma} \exp (-u / \omega)}{\omega^{+} 1 \Gamma\left(\gamma^{+} 1\right)} \quad(\gamma>-1, \omega>0) .
$$

All probability density functions and approximations are limited to $u>0$ in this section, since they are zero for $u<0$; this restriction will be presumed in the remainder of the presentation.

The exceedance distribution function is related to the incomplete Gamma function $[5,6.5 .3]$ :

$$
1-P(u)=\int_{u}^{\infty} d t p(t)=\Gamma(\gamma+1, u / \omega) / \Gamma(\gamma+1) .
$$

The characteristic function follows from (151) as

$$
f(i \xi)=(1-i \xi \omega)^{-\gamma-1},
$$

with cumulants

$$
\chi_{k}=(k-1) !(\gamma+1) \omega^{k} \quad \text { for } k \geq 1, \chi_{0}=0,
$$

and moments

$$
\mu_{k}=\left(\gamma^{+1}\right)_{k}{ }^{\omega^{k}} \text { for } k \geq 0 \text {. }
$$


TR 7377

Thus either set of statistics can be used as a starting position. The error integral in (21) is finite if

$$
-1<\alpha<2 \gamma+1 \text { and } \beta>\omega / 2 \text {. }
$$

We will find the expansion coefficients by means of the characteristic function expansion (100), developed earlier for the generalized Laguerre series. Specifically, we utilize the power series expansion

$$
\begin{aligned}
& (1-w)^{-\alpha-1} f\left(\frac{-w / \beta}{1-w}\right)=(1-w)^{\gamma-\alpha}\left(1-w \frac{\beta-\omega}{\beta}\right)^{-\gamma-1}= \\
& \quad=\sum_{m=0}^{\infty} \frac{(\alpha-\gamma)_{m}}{m !} w^{m} \sum_{k=0}^{\infty} \frac{\left(\gamma^{+1}\right) k}{k !}\left(\frac{\beta-\omega}{\beta}\right)^{k} w^{k},
\end{aligned}
$$

where we used (153) and [5, 15.1.8] twice. The coefficient of a general term $w^{n}$ is then immediately given by the closed form

$$
c_{n}=\sum_{m=0}^{n} \frac{(\alpha-\gamma) m}{m !} \frac{(\gamma+1) n-m}{(n-m) !}\left(\frac{\beta-\omega}{\beta}\right)^{n-m} \text { for } n \geq 0 \text {. }
$$

Alternative expressions for the expansion coefficients are

$$
\begin{aligned}
& c_{n}=\frac{(\gamma+1) n}{n !}\left(\frac{\beta-\omega}{\beta}\right)^{n} \quad F\left(\alpha-\gamma,-n ;-n-\gamma ; \frac{\beta}{\beta-\omega}\right)= \\
& =\frac{(\gamma+1) n}{n !}\left(\frac{-\omega}{\beta}\right)^{n} F\left(-n,-n-\alpha ;-n-\gamma ; \frac{\beta}{\omega}\right)= \\
& =\frac{\left(\alpha^{+} 1\right) n}{n !} F\left(-n, \gamma+1 ; \alpha+1 ; \frac{\omega}{\beta}\right) \quad \text { for } n \geq 0 \text {, }
\end{aligned}
$$

obtained by means of $[5,15.1 .1,15.3 .5,15.3 .7]$ respectively. In fact, the last result can be obtained directly by using $[8,7.4147]$ on (90) and (151): 


$$
c_{n}=\int_{0}^{\infty} d u \frac{u^{\gamma} \exp (-u / \omega)}{\omega^{\gamma^{+}} \Gamma\left(\gamma^{+1}\right)} L_{n}^{(\alpha)}\left(\frac{u}{\beta}\right) .
$$

However, the latter two results in (159) are not numerically stable, whereas (158) and the first line of (159) are stable for large $n$, without encountering round-off error.

Some special cases of (158) are as follows:

$$
\begin{aligned}
& \text { if } \alpha=\gamma \text {, then } c_{n}=\frac{(\gamma+1)_{n}}{n !}\left(\frac{\beta-\omega}{\beta}\right)^{n} ; \\
& \text { if } \beta=\omega \text {, then } c_{n}=\frac{(\alpha-\gamma)_{n}}{n !} ; \\
& \text { if } \alpha=\gamma \text { and } \beta=\omega \text {, then } c_{n}=\delta_{n 0} \text {. }
\end{aligned}
$$

The last case is to be expected, since the weighting exactly matches. the probability density function (151) then.

A numerical example of sequence $\left\{b_{n}\right\}$ for

$$
\gamma=1.1, \quad \omega=2.3 \quad \alpha=1.105, \quad \beta=2.1
$$

is shown in figure 11, using the three recursive procedures developed earlier for the generalized Laguerre series in (112), (119), (126). In addition, exact result (158) is plotted for comparison. The expansion coefficients have a rapidly decaying transient for $n<10$, and then a decay approximately proportional to $n^{-3 / 2}$ for large $n$. The abrupt change of character at $n=5$ does not signify the onset of round-off error; rather, the latter is indicated 


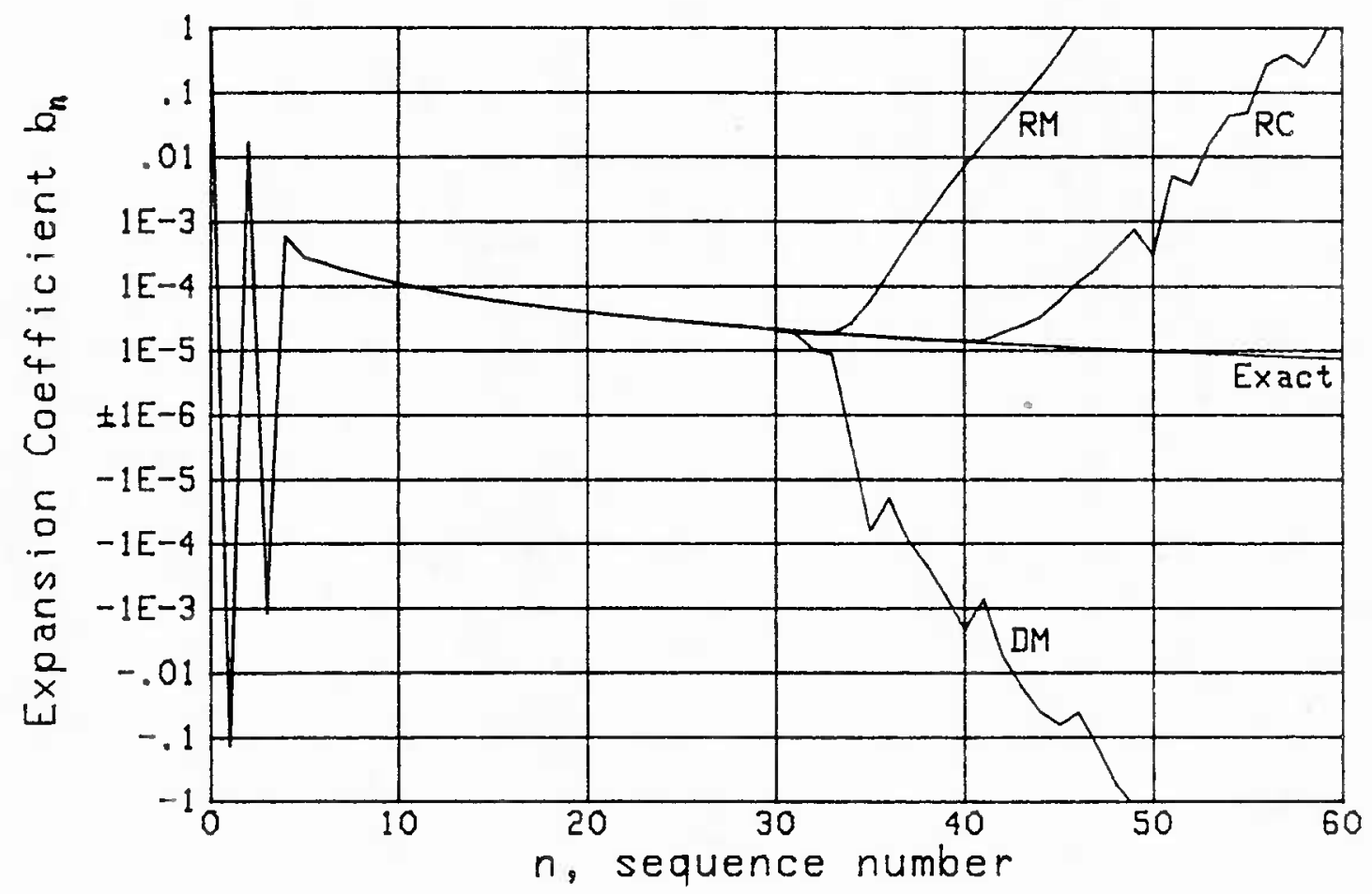

Figure 11. Generalized Laguerre; Example E

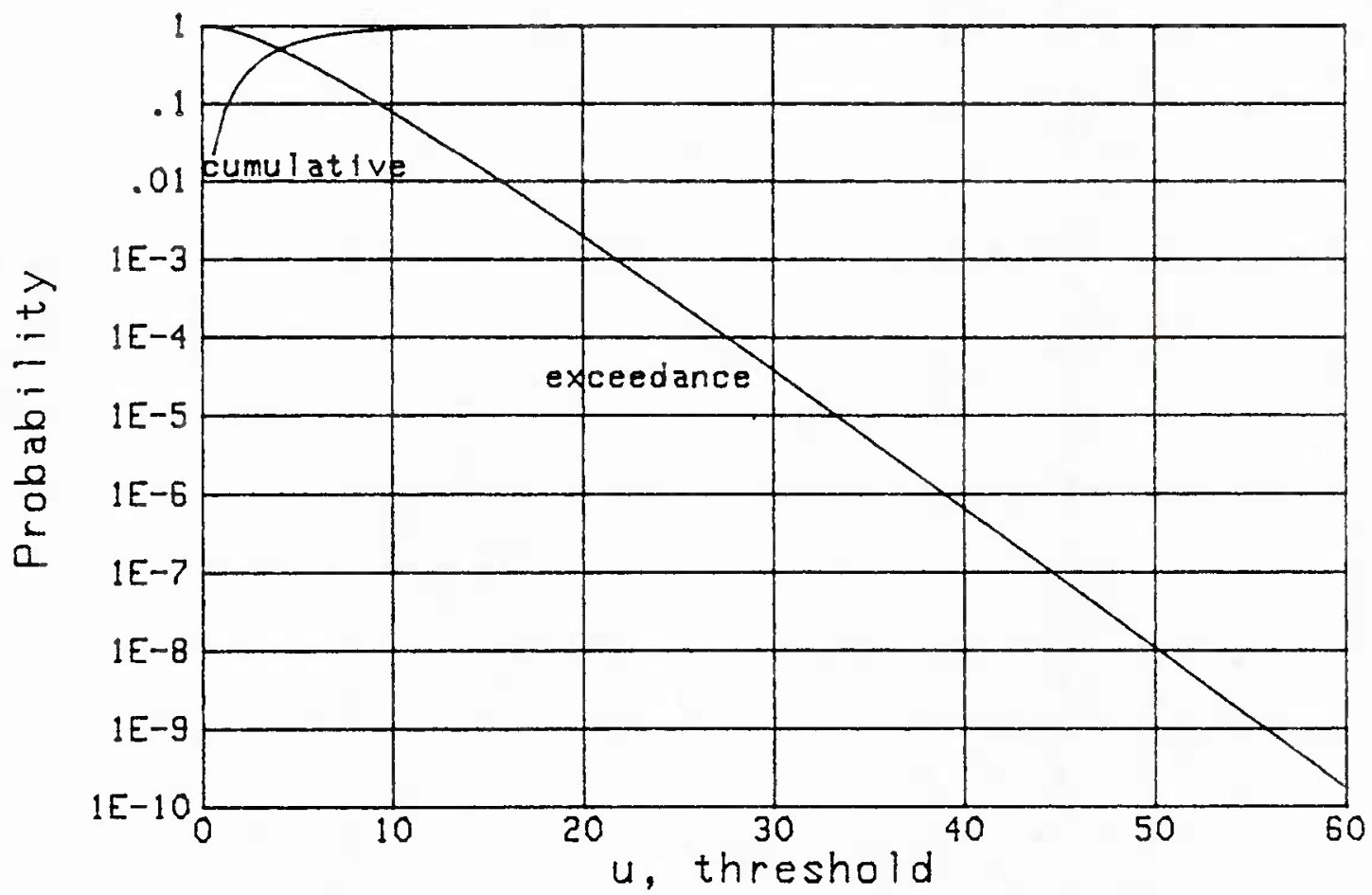

Figure 12. Distributions for Example $E$ 
by an erratic behavior, typically increasing exponentially with $n$ (linear growth on a logarithmic ordinate).

A different plotting strategy will be adopted henceforth for the expansion coefficients $\left\{b_{n}\right\}$, in order not to clutter the diagrams with large oscillations as in figures $1,3,5,7$. Specifically, when the expansion coefficient $b_{n}$ first exceeds the \pm 1 limits, the remainder of sequence $\left\{b_{n}\right\}$ will not be plotted, since this is a region of large round-off error. Thus, although the $R M$ curve in figure 11 returns to the \pm 1 limits briefly at $n=52,53$, these values are not displayed.

Round-off error for the RC procedure does not become as significant as for the two moment approaches until $n$ has increased by almost 10 , for this example in figure 11. In fact, the expansion coefficients for the RC procedure overlap the exact values until $n=40$. The corresponding approximate distributions, using $N=40$ terms in expansion (95) as determined by $\mathrm{RC}$, are plotted in figure 12 . The exact result (152) overlays these results over the entire range plotted.

EXAMPLE F

The following probability density function corresponds to a noncentral Chi-square variate of $2 v$ degrees of freedom:

$$
p(u)=\frac{1}{2} \exp \left(-\frac{d^{2}+u}{2}\right)\left(\frac{\sqrt{u}}{d}\right)^{v-1} I_{v-1}(d \sqrt{u}) \quad(v>0) ;
$$


TR 7377

$d$ is the noncentrality parameter, and $2 v$ need not be integer. The characteristic function is $[8,6.6314]$

$$
f(i \xi)=(1-i 2 \xi)^{-\nu} \exp \left(\frac{i d^{2} \xi}{1-i 2 \xi}\right) \text {, }
$$

and is the same as the one considered in $[10,(50)$ et seq.]. The exceedance distribution function is the generalized Q-function:

$$
\begin{aligned}
1-P(u) & =\int_{u}^{\infty} d t \frac{1}{2} \exp \left(-\frac{d^{2}+t}{2}\right)\left(\frac{\sqrt{t}}{d}\right)^{v-1} I_{v-1}(d \sqrt{t})= \\
= & \int_{\sqrt{u}}^{\infty} d x x \exp \left(-\frac{d^{2}+x^{2}}{2}\right)\left(\frac{x}{d}\right)^{v-1} I_{v-1}(d x)=Q_{v}(d, \sqrt{u}) .
\end{aligned}
$$

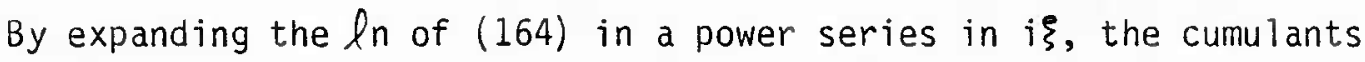
follow as

$$
\chi_{n}=2^{n}(n-1) !\left(v+\frac{1}{2} d^{2} n\right) \quad \text { for } n \geq 1, \quad \chi_{0}=0
$$

And the moments are obtained from (163) as

$$
\begin{aligned}
\mu_{n} & =2^{n}(v){ }_{n} 1_{1} F_{1}\left(-n ; v ;-d^{2} / 2\right)= \\
& =2^{n} n ! L_{n}^{(v-1)}\left(-d^{2} / 2\right) \text { for } n \geq 0,
\end{aligned}
$$

by use of $[8,6.6311]$ and $[5,13.6 .9]$. Both (166) and (167) lend themselves to simple recurrences which involve only positive quantities; thus the starting statistics can be quickly and accurately evaluated.

The numerical example we consider here will be compared with the exact results in [10, figure 11$]$, namely, 


$$
v=2.7, \quad d=3 \quad \alpha=1.7, \quad \beta=5.5 \text {. }
$$

Since the probability density function in (163) behaves as $u^{\nu-1}$ as $u \rightarrow 0^{+}$, it is reasonable to choose weighting parameter $\alpha$ in (82) as $v-1$, as indicated in (168). And since (163) behaves as $\exp (-u / 2)$ as $u \rightarrow+\infty$, we must choose B > 1 in order that the error integral in (21) is finite. The particular values in (168) approximateiy minimize the sum of $\left\{b_{n}^{2}\right\}_{0}^{N}$ in (21).

The expansion coefficients $\left\{b_{n}\right\}$ as determined by the three available recursive procedures are displayed in figure 13. The RC coefficients decrease to values less than $1 E-10$ near $n=50$, before round-off error becomes significant. The two moment approaches deteriorate near $\mathrm{n}=30$, which is markedly poorer than the cumulant approach. The distributions, as determined by $N=50$ terms of the $R C$ approach, are given in figure 14, and agree with the $d=3$ curve of $[10$, figure 11$]$. When the approximate probability density function for $N=50$ was compared with exact result (163), 10 decimals of agreement were obtained; this is due to the ability to get very small $\left\{b_{n}\right\}$ in figure 13 via the $R C$ method.

EXAMPLE G

This example is the Rice probability density function given in (144), with moments (145) and cumulative distribution function (146). The starting statistics are the moments as determined by recurrence $(E-5)-(E-6)$. 


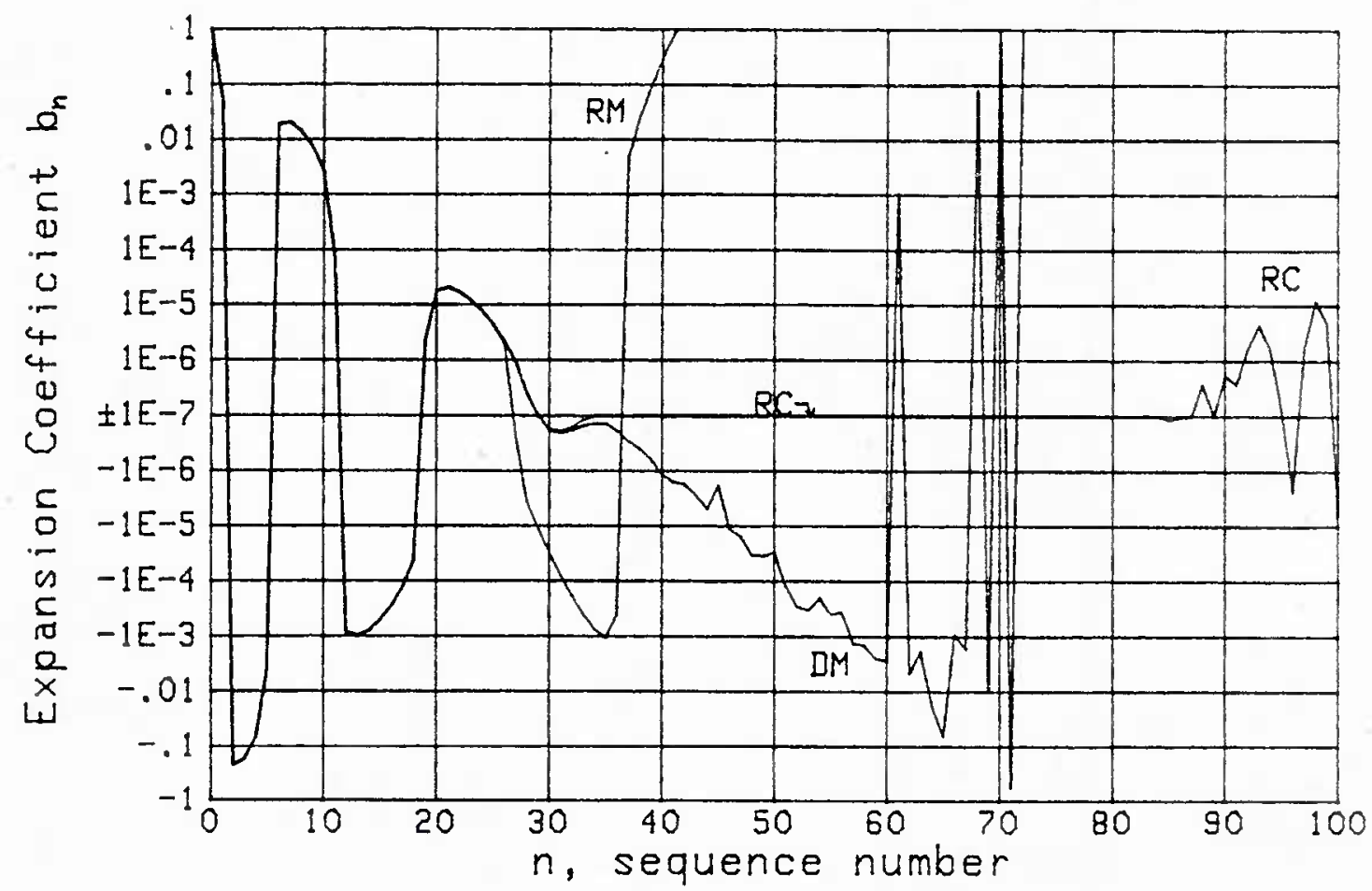

Figure 13. Generalized Laguerre; Example F

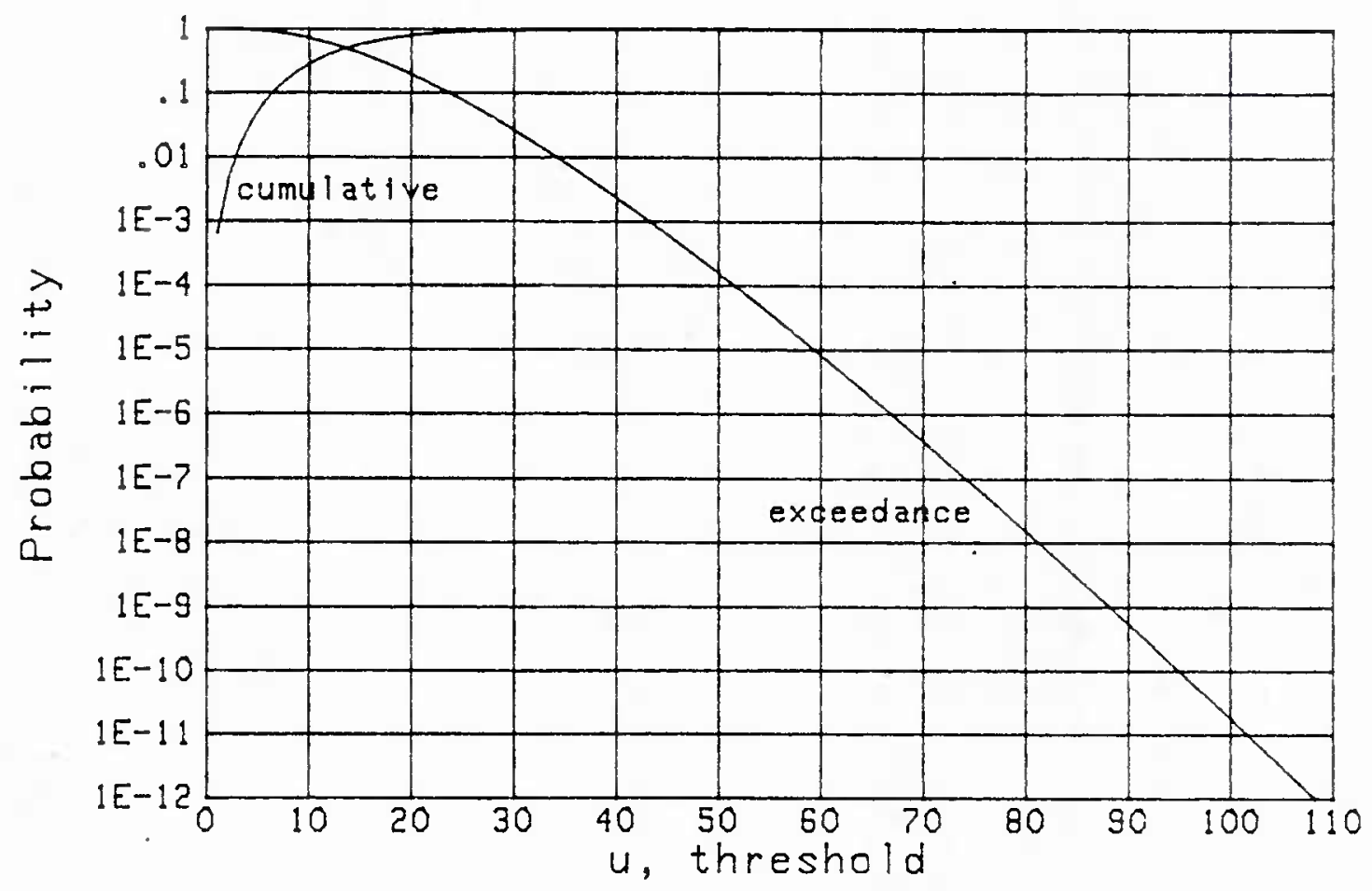

Figure 14. Distributions for Example $F$ 
The particular numerical case of interest is

$$
\theta=3, \quad \omega^{2}=2 \quad \alpha=1, \quad \beta=1 \text {. }
$$

The values of $\alpha$ and $\beta$ were found by the usual trial and error search procedure of observing plots of expansion coefficients $\left\{b_{n}\right\}$, looking for rapid decay and small round-off error; results for this example are displayed in figure 15. The $R M$ procedure deteriorates rapidly at $n=30$, whereas $D M$ and $R C$ are useable up to $\mathrm{n}=55$ and 65 approximately.

The cumulative and exceedance distribution functions for $N=65$ terms of the $\mathrm{RC}$ procedure are plotted in figure 16, along with exact result (146). The approximate exceedance distribution function overlaps the exact one until slightly below the probability level $1 E-4$, which corresponds to the level of reliability of $b_{n}$ in figure 15 at $n=65$. Then the exceedance distribution function makes a positive (upward) turn below $1 E-6$, which is impossible for a physical density function which must remain positive; thus the approximation deteriorates rapidly for $u>7$.

EXAMPLE H

This is a follow-on to the previous example, in that we consider a sum of 8 Rice variates, each with the statistics in (169). The expansion coefficients for

$$
\theta=3, \quad \omega^{2}=2 \quad \alpha=26, \quad \beta=1
$$




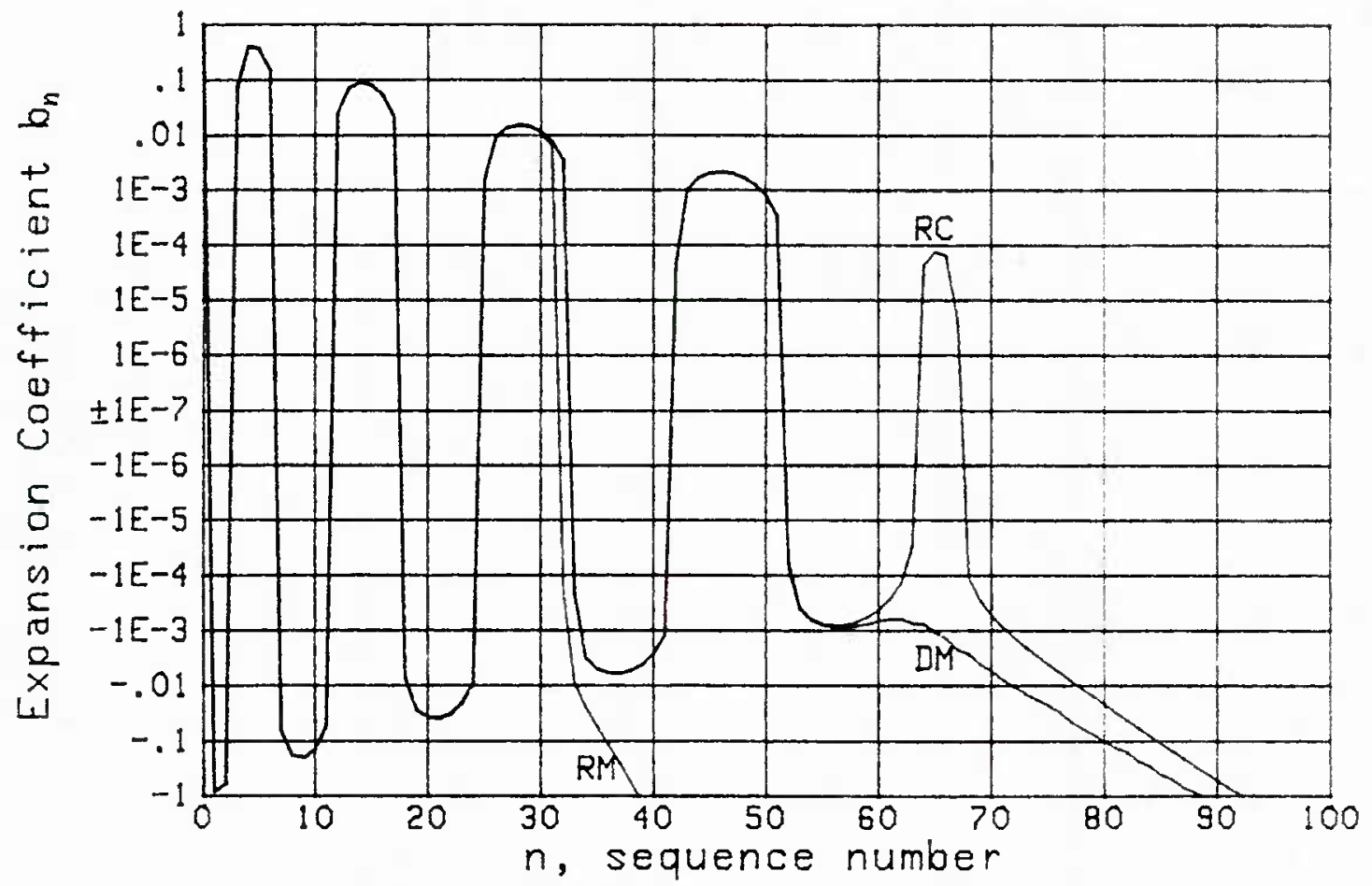

Figure 15. Generalized Laguerre; Example $G$

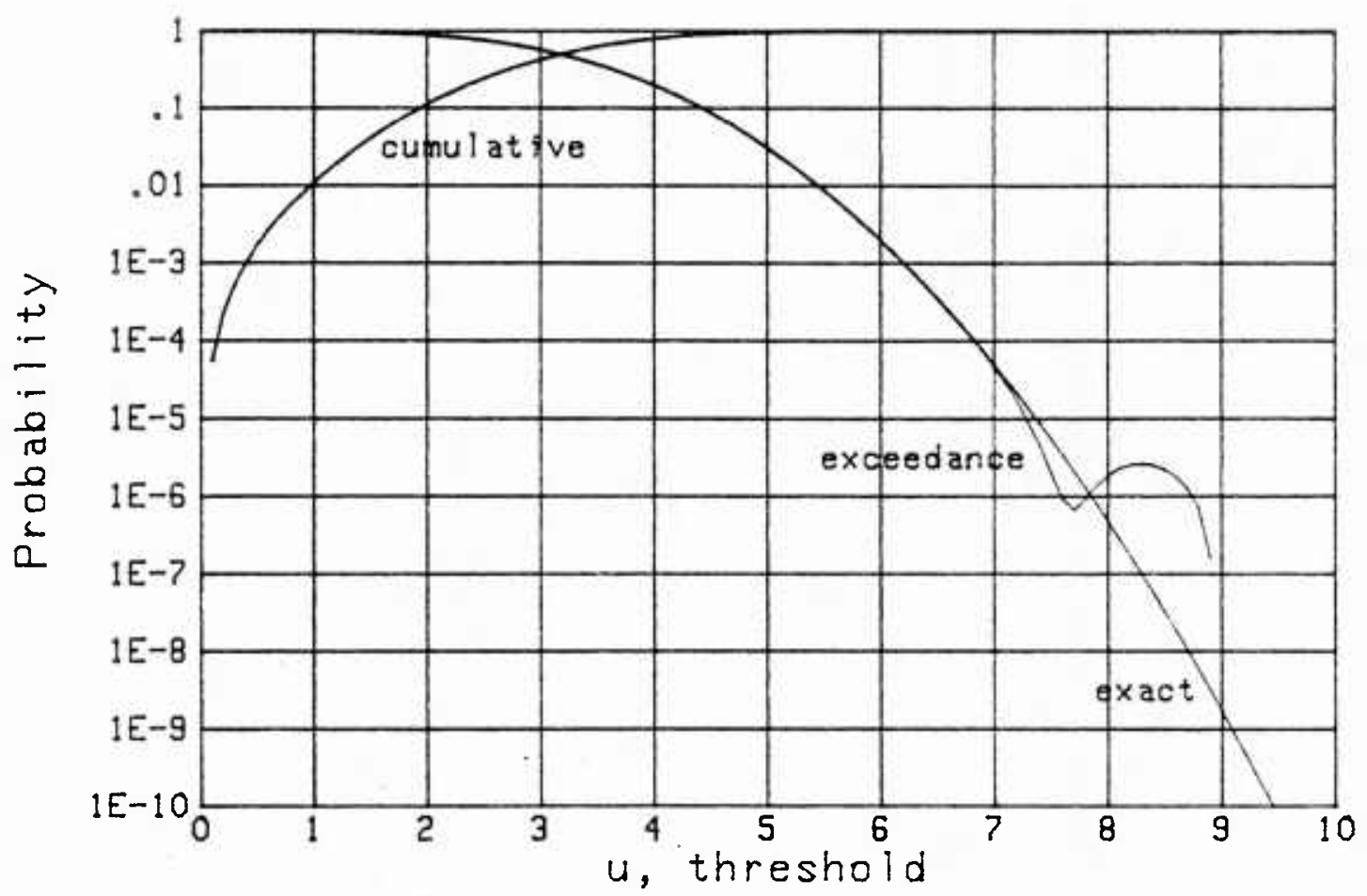

Figure 16. Distributions for Example $G$ 
are displayed in figure 17. Whereas both $D M$ and $R M$ are useless beyond $n=25$, the expansion coefficients determined by RC decay down to the $1 E-10$ level at $n \simeq 150$ before round-off error becomes significant. The corresponding distributions in figure 18, using $N=143$ terms of the expansion via $R C$, reveal accurate results down to the $1 E-12$ level of probability, except for a slight flare in the exceedance distribution function below $1 E-11$.

We also checked the example of the sum of 8 normalized Rayleigh variates considered earlier via a Hermite series in example $C$. For $\alpha=10, \beta=.9$, the expansion coefficients $\left\{b_{n}\right\}$ decayed to the $1 E-11$ level at $n=100$ for the $R C$ approach and agreed with the false alarm probabilities calculated exactly in $[9$, table 1] for $M=8$. By contrast, the $D M$ expansion coefficients were subject to significant round-off error by the time $n$ reached 30 , and were useless for small probability calculations.

\section{EXAMPLE I}

We return to the shot noise process previously considered via a Hermite series in example D. The equations and discussions there should be reviewed, since they are directly relevant to the generalized Laguerre expansion here. For the choice of parameters in (150), the selection of generalized Laguerre weighting parameters

$$
\alpha=.74, \quad \beta=2.1
$$

leads to the expansion coefficients plotted in figure 19. The DM and RC results agree to $n=32$, and then begin to diverge from each other. By way of 


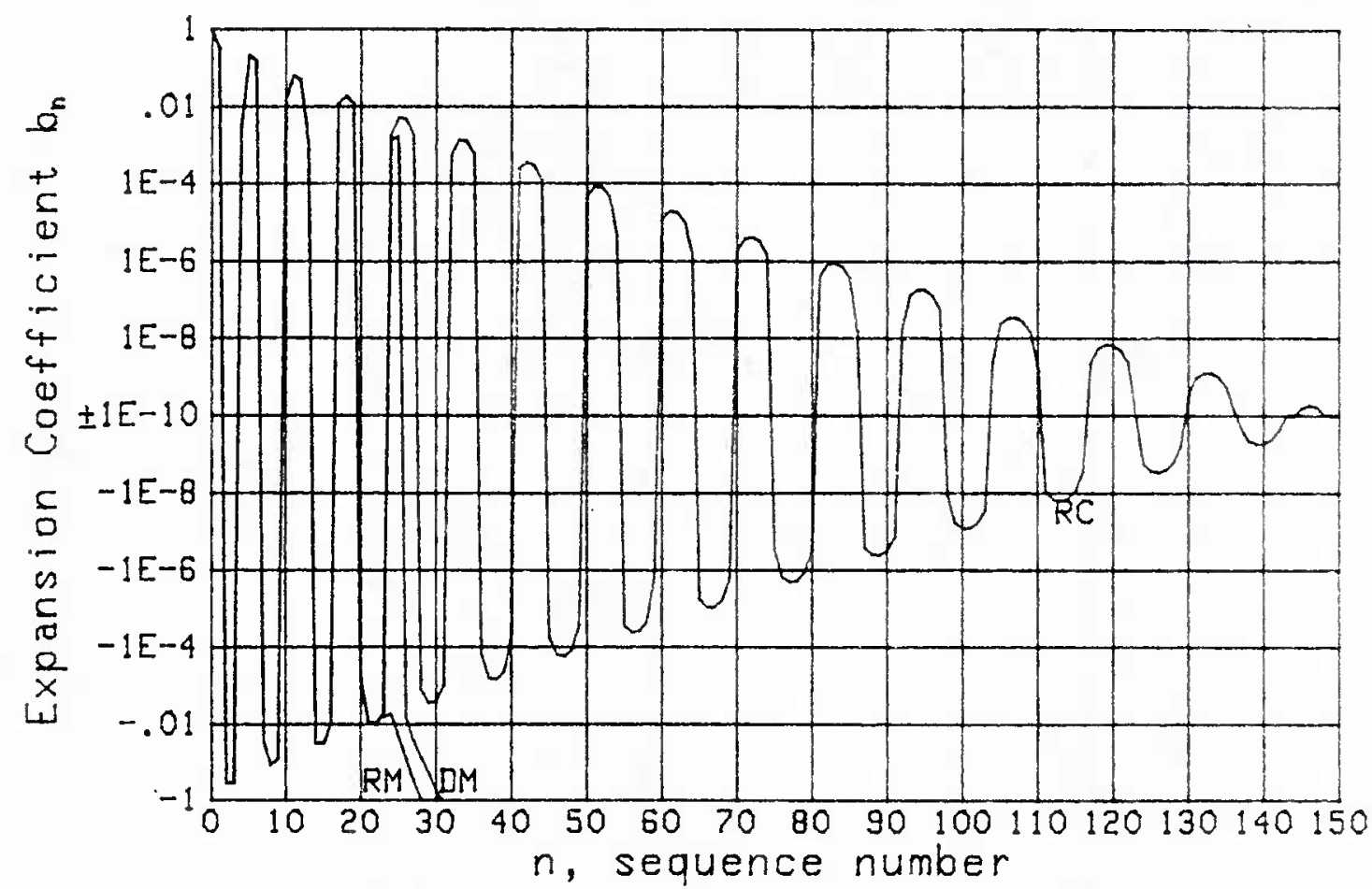

Figure 17. Generalized Laguerre; Example H

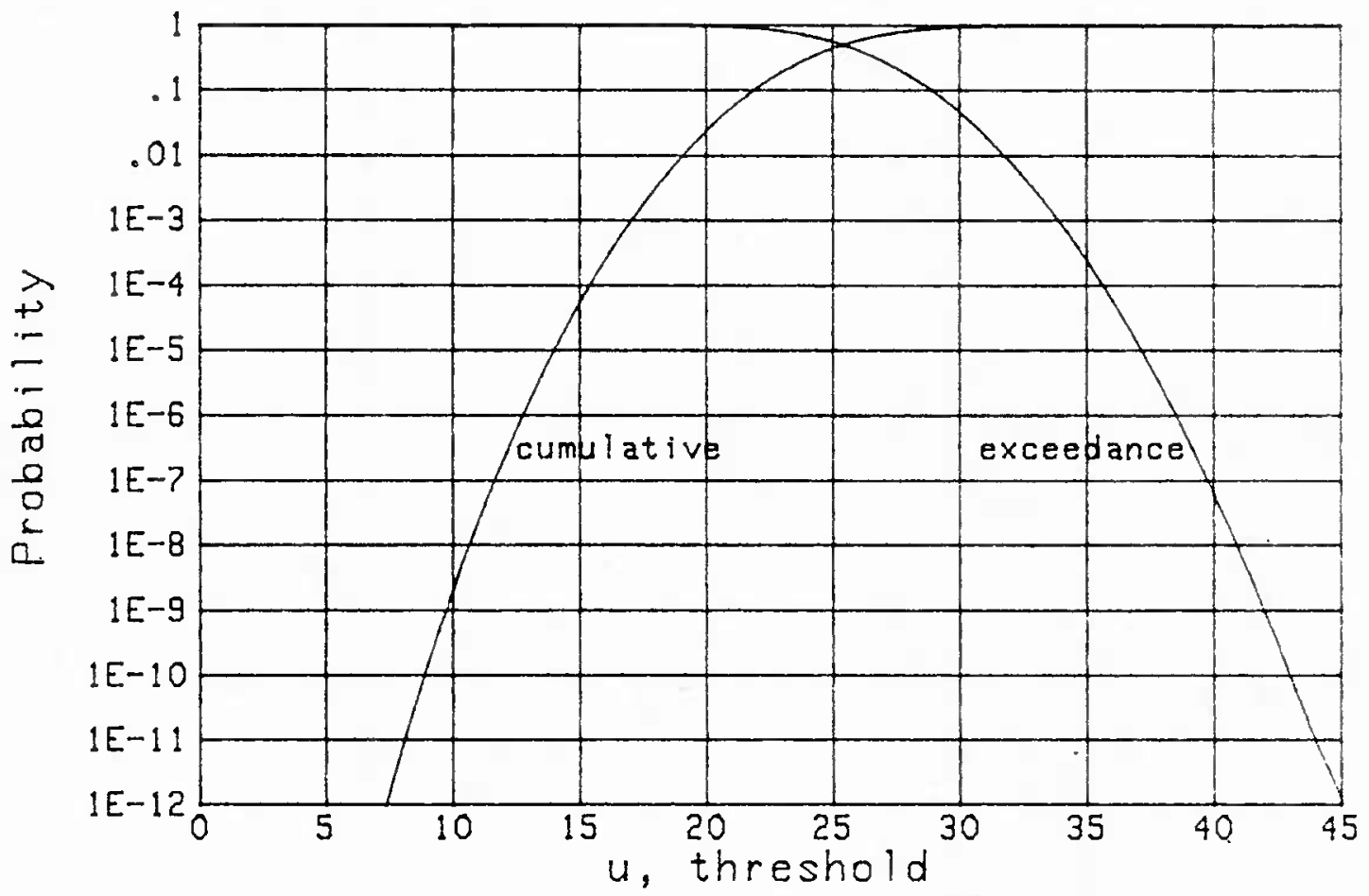

Figure 18. Distributions for Example $H$ 


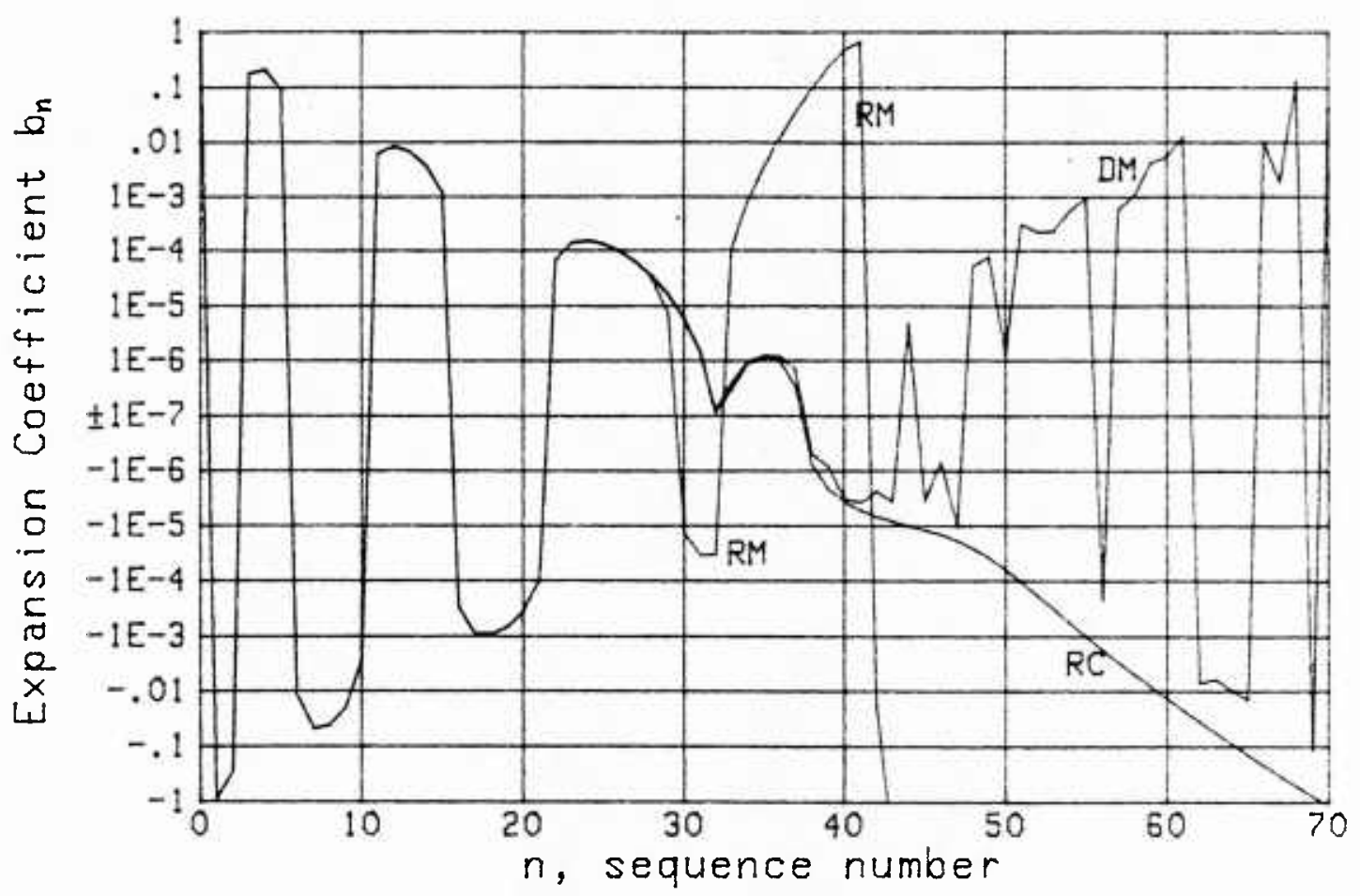

Figure 19. Generalized Laguerre; Example I

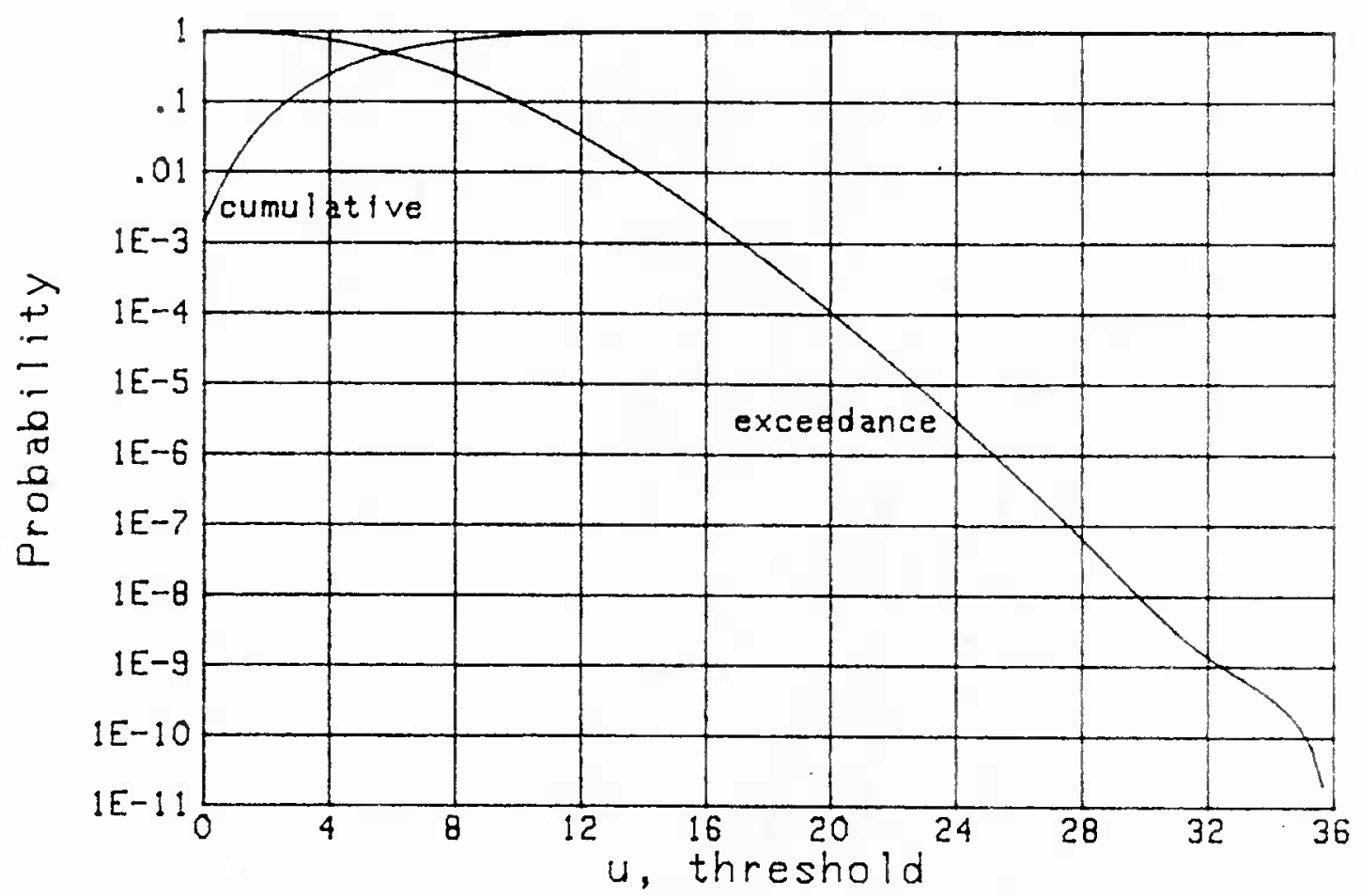

Figure 20. Distributions for Example I 
TR 7377

contrast with the Hermite expansion coefficients in figure 7, where values in the $1 E-3$ range were achieved, values in the $1 E-6$ range can be obtained here for the generalized Laguerre expansion, for $n$ in the mid-30s. The DM result was previously given in [4, figure $0-1]$.

The distributions for $N=32$ terms of the $R C$ procedure are plotted in figure 20. This result is considerably better than the Hermite expansion in figure 8; instead of the wiggles which developed at $1 E-4$ in figure 8 , the curve in figure 20 is smooth down to the $1 E-8$ probability level, and then develops a bump. Also, the cumulative distribution function is accurate at $u=0$, where it takes on the value $P_{0}=.002$ given in $(150)$, and is zero for $u<0$. This cumulative distribution function was previously given in $[4$, figure 8$]$.

The probability density function for $N=32$ terms of the $R C$ procedure is given in figure 21 ; this result was previously given in [4, figure 9]. It is significantly better near the origin than the Hermite approximation given earlier in figure 9, which developed negative values for $u<0$. In order to see what the probability density function does for larger $u$ values, the same probability density function is plotted on a logarithmic ordinate in figure 22. It is accurate to the $1 E-9$ level but then develops a hook that is incorrect; however, this approximation remains positive even at this very low value of the density, whereas the corresponding result via a Hermite expansion in figure 10 developed negative values. The estimated errors in figures 20 and 22 are evaluated in a later section. 
TR 7377

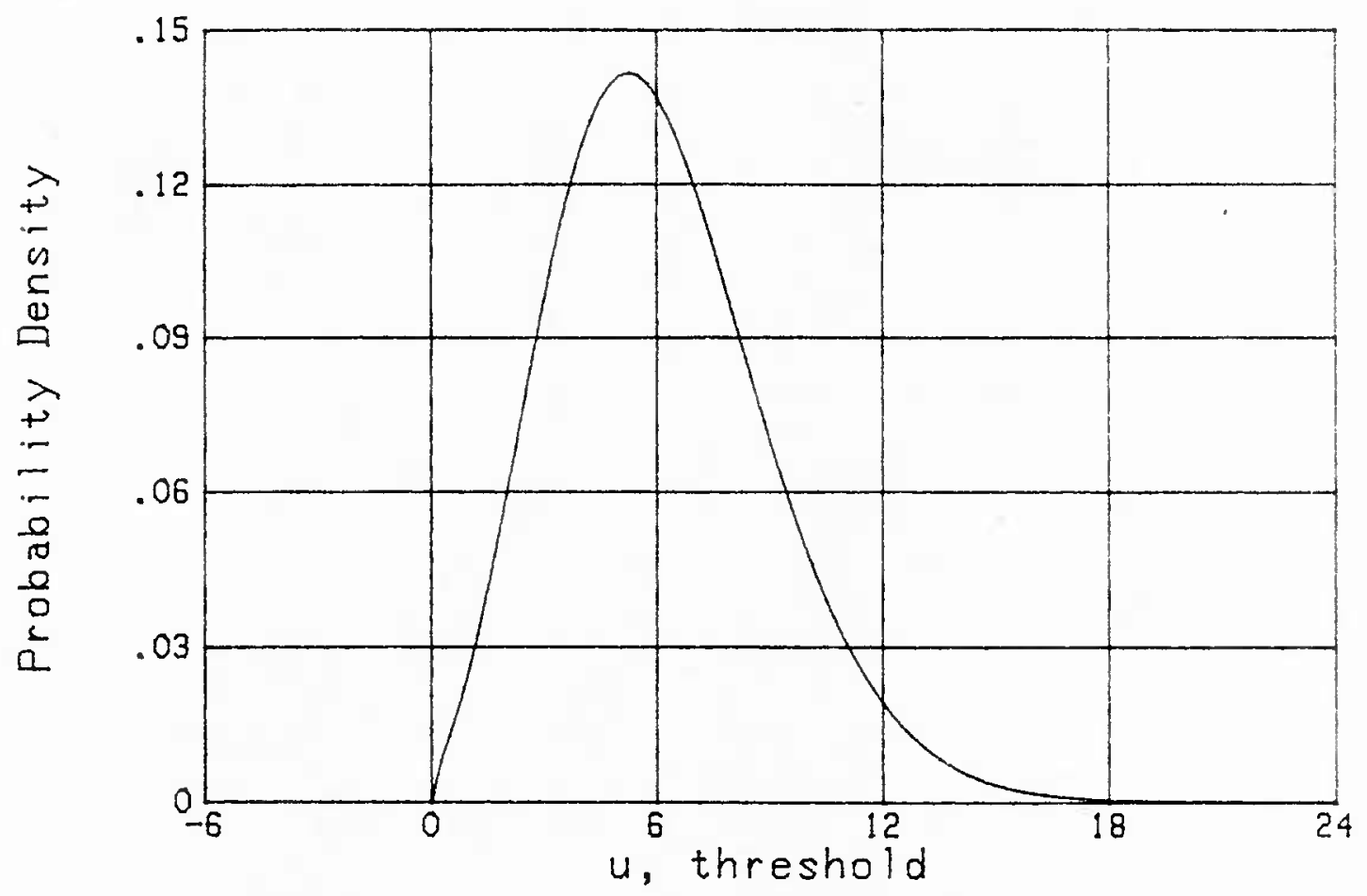

Figure 21. Linear Density for Example I

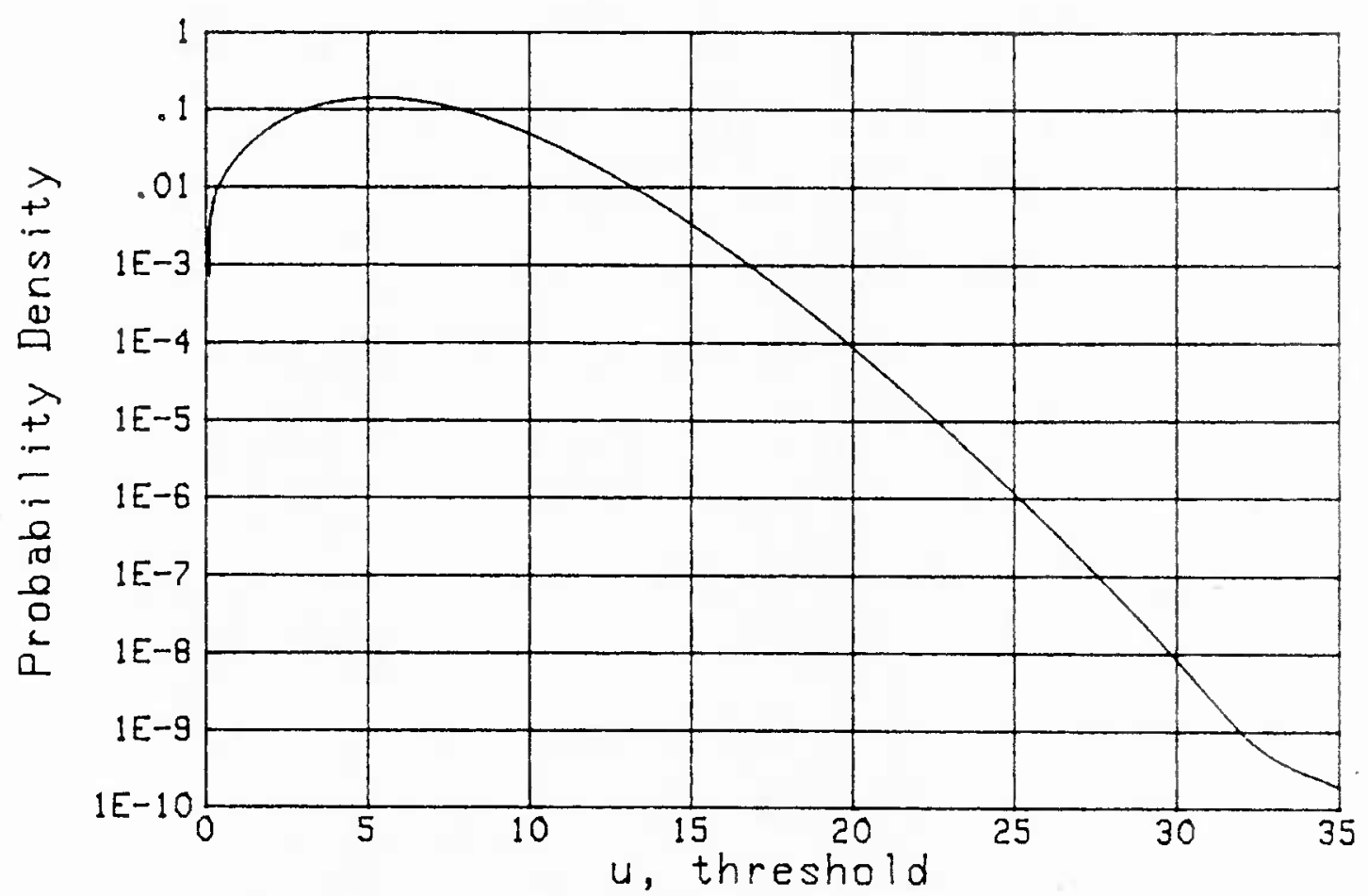

Figure 22. Log Density for Example I 
TR 7377

EXAMPLE $J$

This last example is for probability density function

$$
p(u)=\frac{1}{2} \exp \left(-u^{1 / 2}\right) \text { for } u>0,
$$

for which the moments are

$$
\mu_{n}=(2 n+1) ! \text {. }
$$

The characteristic function and cumulants are not available in any convenient analytic form.

This is a particularly difficult example, since the characteristic function expansion in (6) has a zero radius of convergence; thus the moments do not uniquely determine the probability density function or cumulative distribution function. Aiso, the error integral in (21) is always infinite; in fact, regardless of the choice of weighting parameters $\alpha$ and $\beta$ used in the generalized Laguerre series, the expansion coefficients $\left\{b_{n}\right\}$ always diverged. Nevertheless, a search of parameter values led to a pair of selections, namely,

$$
\alpha=-.35, \quad \beta=30,
$$

for which the expansion coefficients had an initial decay to the $1 E-2$ level before divergence took over; see figure 23. In fact, the identical same results were obtained for all three methods, RC, DM, RM; this is probably due to the fact that divergence of $\left\{b_{n}\right\}$ dominated before round-off error became significant. 


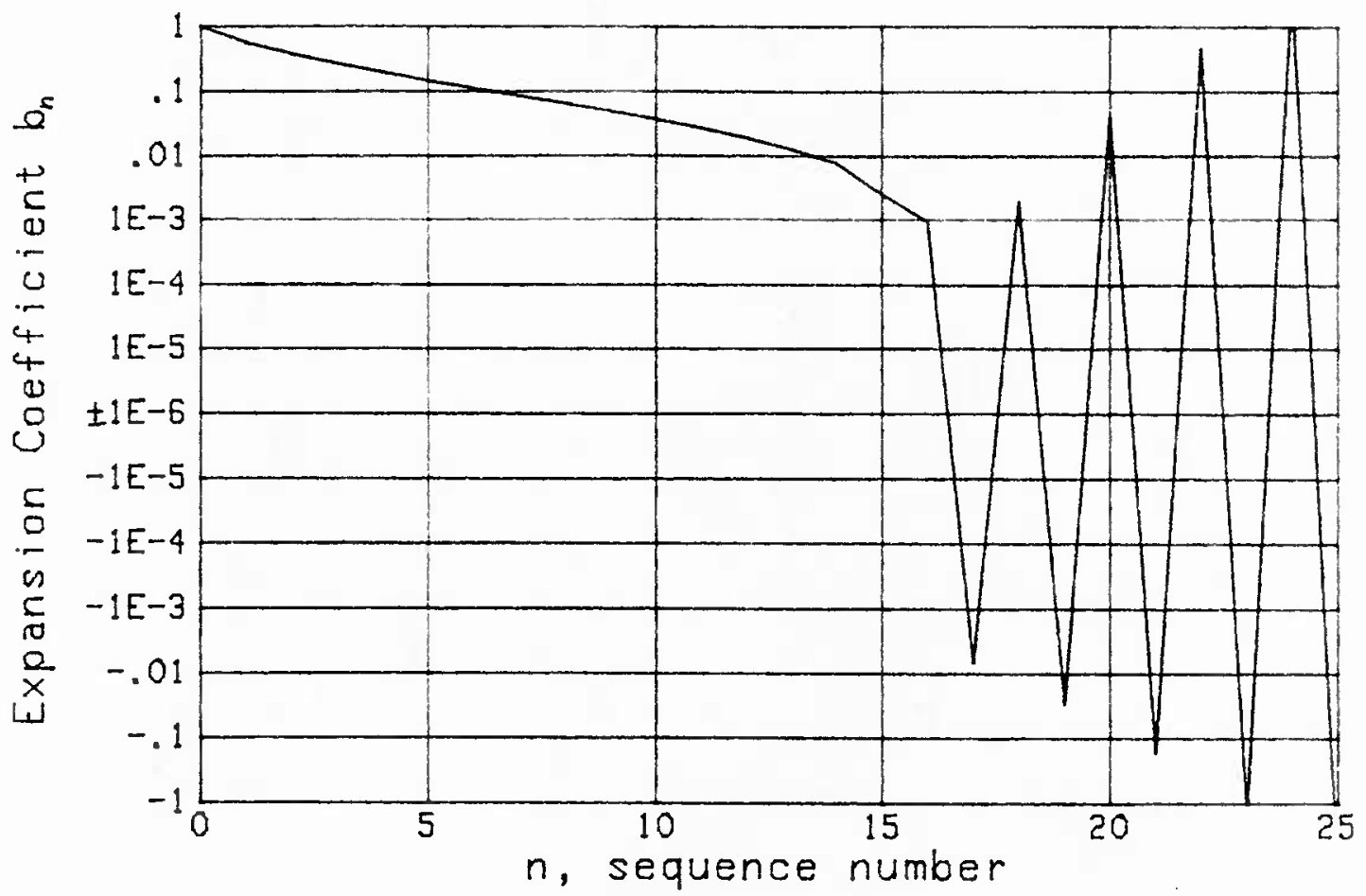

Figure 23. Generalized Laguerre; Example J

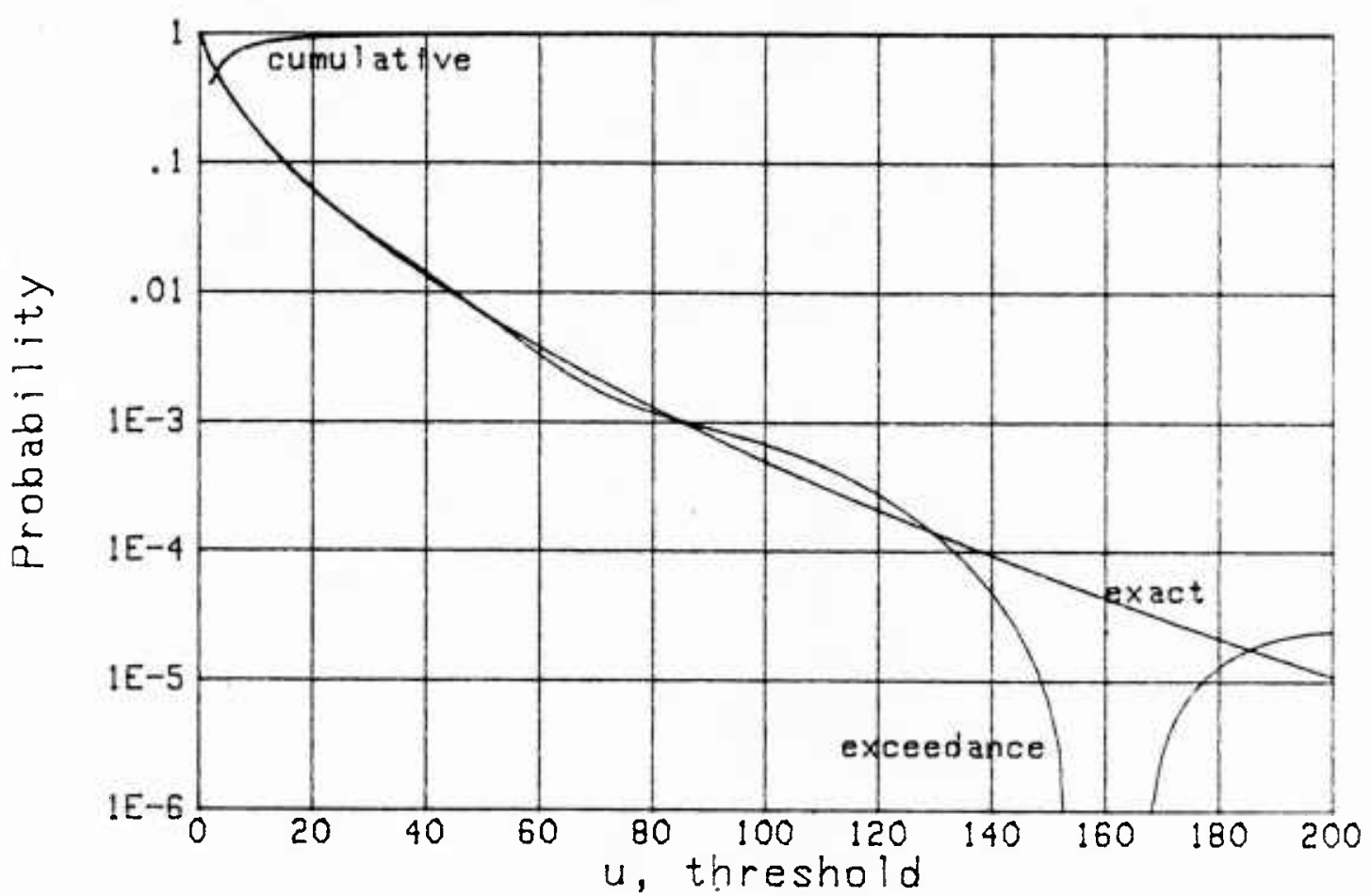

Figure 24. Distributions for Example J 
The distributions are plotted in figure 24 for $N=15$ terms of the generalized Laguerre series. Comparison with the exact exceedance distribution function

$$
1-P(u)=\left(1+u^{1 / 2}\right) \exp \left(-u^{1 / 2}\right) \text { for } u>0
$$

reveals that the approximation is decent down to the .01 probability level, but then oscillates more and more violently as $u$ increases. Thus even in this non-unique example, a limited-quality approximation is achieved by the generalized Laguerre series; this example confirms the comment in [3, p. 167] that, even for a divergent series, a limited number of expansion coefficients often gives a satisfactory approximation.

The exact and approximate probability density functions are plotted on a linear ordinate in figure 25, and on a logarithmic ordinate in figure 26 , using $N=15$ terms of the generalized Laguerre series, when the expansion coefficients were determined by the DM method. The approximate probability density function is negative for $150<u<190$, around the $1 E-6$ level. The estimated errors of the approximations in figures 24 and 26 will be developed in the next section. 
TR 7377

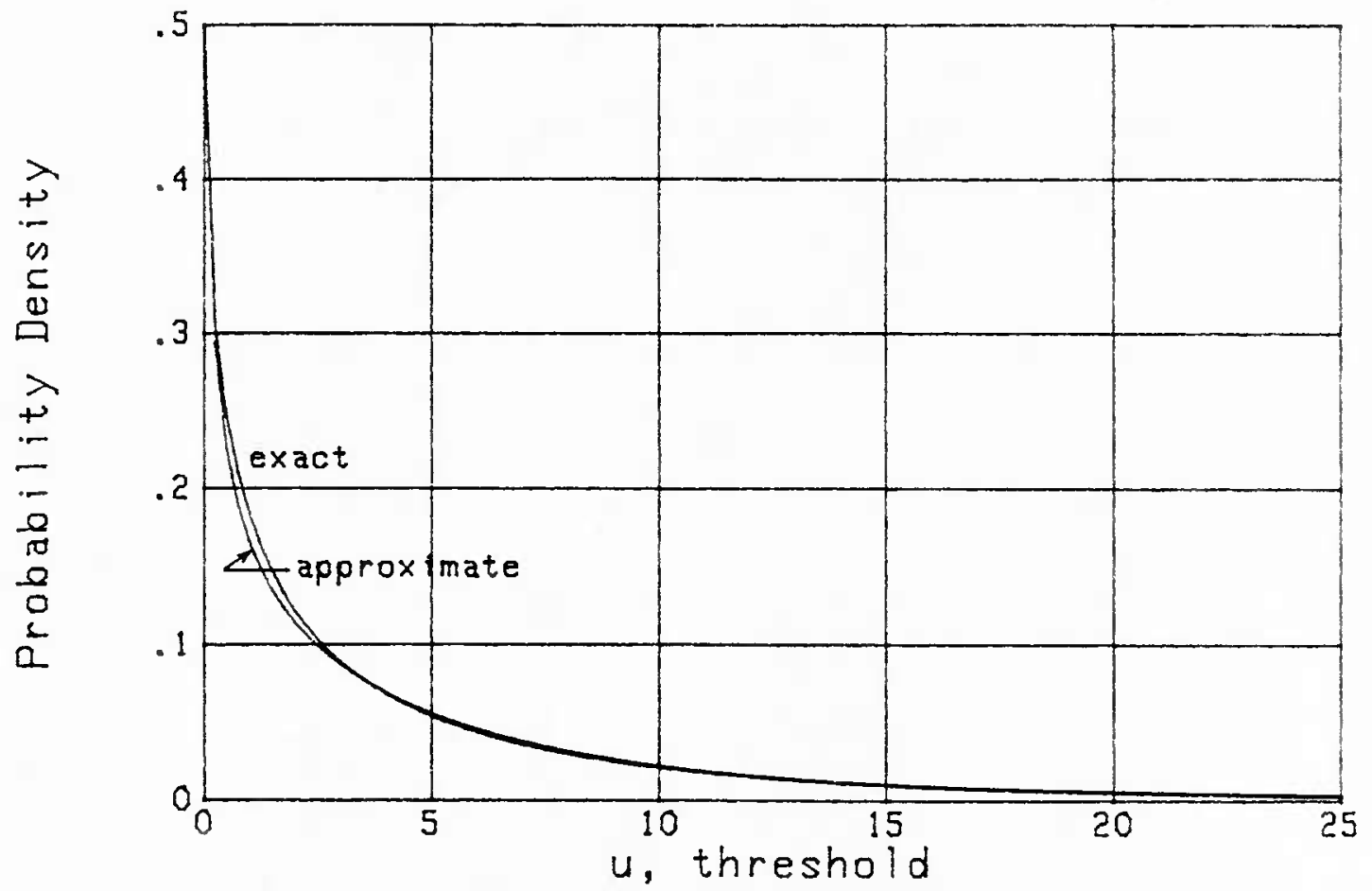

Figure 25. Linear Density for Example J

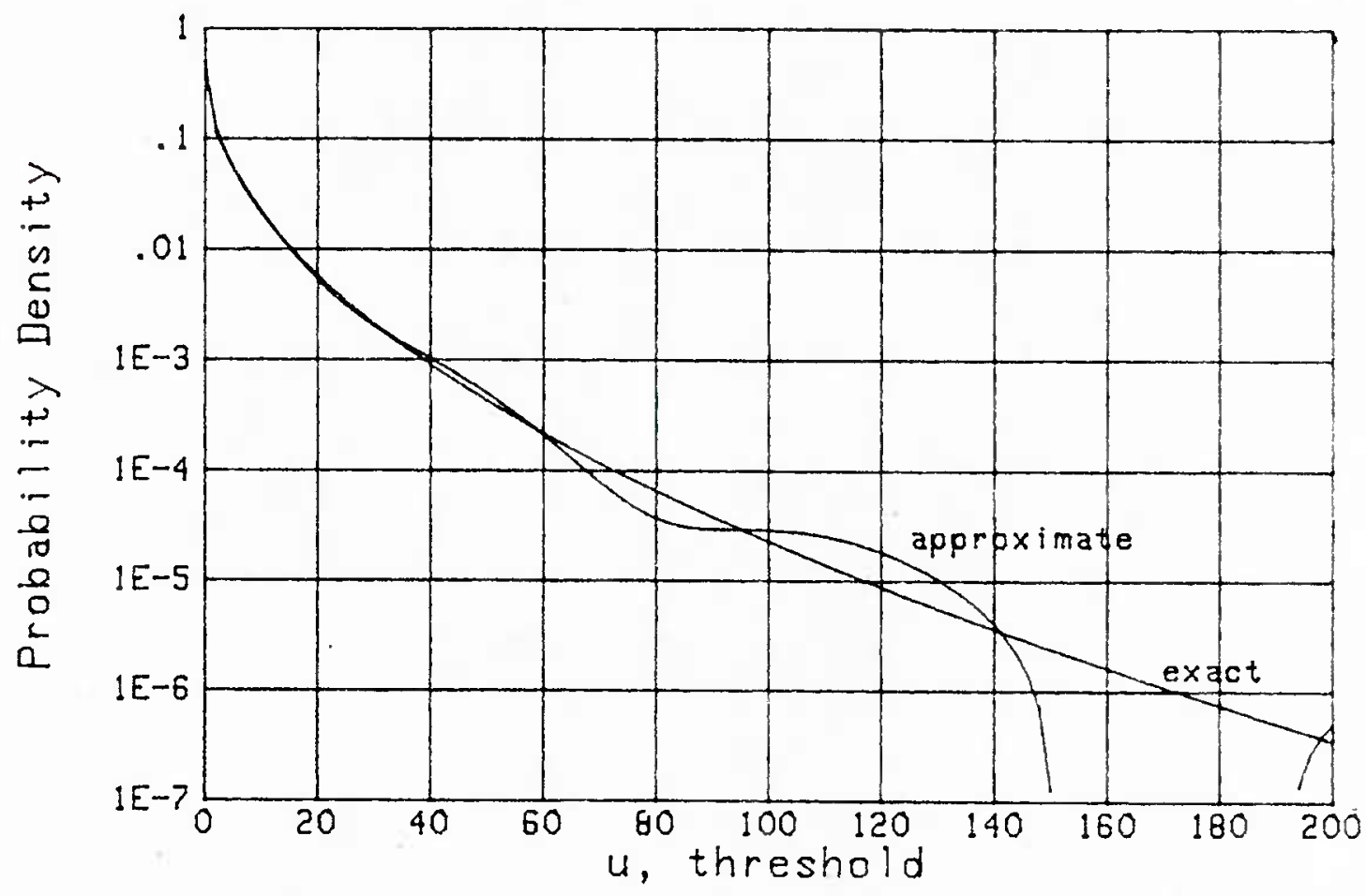

Figure 26. Log Density for Example J 
TR 7377

EST IMATED ERRORS OF APPROXIMATIONS

When the calculations of the approximate cumulative or exceedance distribution functions or the corresponding probability density function are made, it would be very useful to have a rough estimate of their reliability. One way, as discussed in the previous sections, is to look for nonsmooth or anomalous behavior on the tails of the functions. Here, we will develop a more quantitative estimate of the error and superpose it on some of the previous examples, for confirmation.

Both the Hermite and generalized Laguerre orthonormal polynomials oscillate with $n$ and decay slowly. The same general behavior is true of expansion coefficients $\left\{b_{n}\right\}$. This leads to summations for the various functions with terms that also oscillate and decay. A rough estimate of the error is afforded by the envelope of these oscillations, evaluated at the first neglected term of the summation. This procedure will be pursued for both types of expansions; how useful it is will be indicated by numerical examples.

HERMITE EXPANSION

The following result for the envelope of the Hermite polynomial is obtained from $[5,6.1 .39$ and 22.5 .18$]$ and $[7,8.22 .8]$ :

$$
\operatorname{Env}\left\{(n !)^{-1 / 2} H_{n}(x)\right\} \sim \exp \left(x^{2} / 4\right)\left(\frac{2}{\pi n}\right)^{1 / 4} \text { as } n \rightarrow+\infty
$$

Also, from (46) and (47), the $n$-th term of the approximate probability density function is 


$$
\frac{1}{\beta} \phi\left(\frac{u-\alpha}{\beta}\right) b_{n}(n !)^{-1 / 2} H_{n}\left(\frac{u-\alpha}{\beta}\right) \text {. }
$$

Then the magnitude of the error of the probability density function approximation, if the $n$-th term is the first one neglected, is roughly

$$
\begin{aligned}
& E_{n}(u ; p) \equiv \frac{1}{\beta} \phi\left(\frac{u-\alpha}{\beta}\right) \operatorname{Env}\left\{b_{n}\right\} \operatorname{Env}\left\{(n !)^{-1 / 2} \operatorname{He}_{n}\left(\frac{u-\alpha}{\beta}\right)\right\}= \\
& \sim\left[2^{1 / 4} \pi^{3 / 4}\right]^{-1} \exp \left(-\frac{(u-\alpha)^{2}}{4 \beta^{2}}\right) n^{-1 / 4} \operatorname{Env}\left\{b_{n}\right\} \text { as } n \rightarrow+\infty
\end{aligned}
$$

Here we used (176).

As for the cumulative distribution function, we have from (47)-(49), the $\mathrm{n}$-th term of the approximation as

$$
-\phi\left(\frac{u-\alpha}{\beta}\right) b_{n}(n !)^{-1 / 2} H e_{n-1}\left(\frac{u-\alpha}{\beta}\right) .
$$

The magnitude of the error for the cumulative and exceedance distribution functions if the $n$-th term is the first one neglected, is then defined as

$$
\begin{aligned}
& E_{n}(u ; P) \equiv \emptyset\left(\frac{u-\alpha}{\beta}\right) \operatorname{Env}\left\{b_{n}\right\} \operatorname{Env}\left\{(n !)^{-1 / 2} H_{n-1}\left(\frac{u-\alpha}{\beta}\right)\right\}= \\
& \sim\left[2^{1 / 4} \pi^{3 / 4}\right]^{-1} \exp \left(-\frac{(u-\alpha)^{2}}{4 \beta^{2}}\right) n^{-3 / 4} \operatorname{Env}\left\{b_{n}\right\} \text { as } n \rightarrow+\infty .
\end{aligned}
$$

Again, (176) was of crucial importance in getting this result. 
TR 7377

Since the above estimates are asymptotic in $n$, they will be most reliable for $n$ large; their use for small $n$ could be very misleading. The way to use these error estimates for the density and distribution approximations is as follows. First, a search on $\alpha$ and $\beta$, to find the fastest decaying expansion coefficients $\left\{b_{n}\right\}$, is conducted. The weighting parameter values, $\alpha$ and $\beta$, and the corresponding envelope value of the expansion coefficients $\left\{b_{n}\right\}$ at the point, $n$, where round-off error becomes dominant, are then noted. (For example, for figure 7, we observe that $E_{n v}\left\{b_{n}\right\} \approx 2 E-3$ at $n=65$, when $\alpha=6.1, \beta=4.3$; see example D.) Then (178) and (180) can be computed and plotted in the ranges of $u$ of interest.

An example of this procedure for the shot noise process in example $D$ is given in figures 27 and 28. In particular, the approximate results are repeated from figures 8 and 10, and error measures (180) and (178), respectively, are superposed as dashed lines, each on the appropriate figure. Just where the approximations develop large wiggles, the errors are of comparable magnitude, indicating unreliable estimates there.

It should be observed from these figures (or from (178) and (180)) that the absolute error is maximum at $u=\alpha$, but that the relative error is a minimum in that neighborhood. Also, although the absolute error decays with $u$, the correct answer decays faster, leading to an increasing relative error, which eventually becomes so excessive in the tails of the various functions that the approximations are useless. 


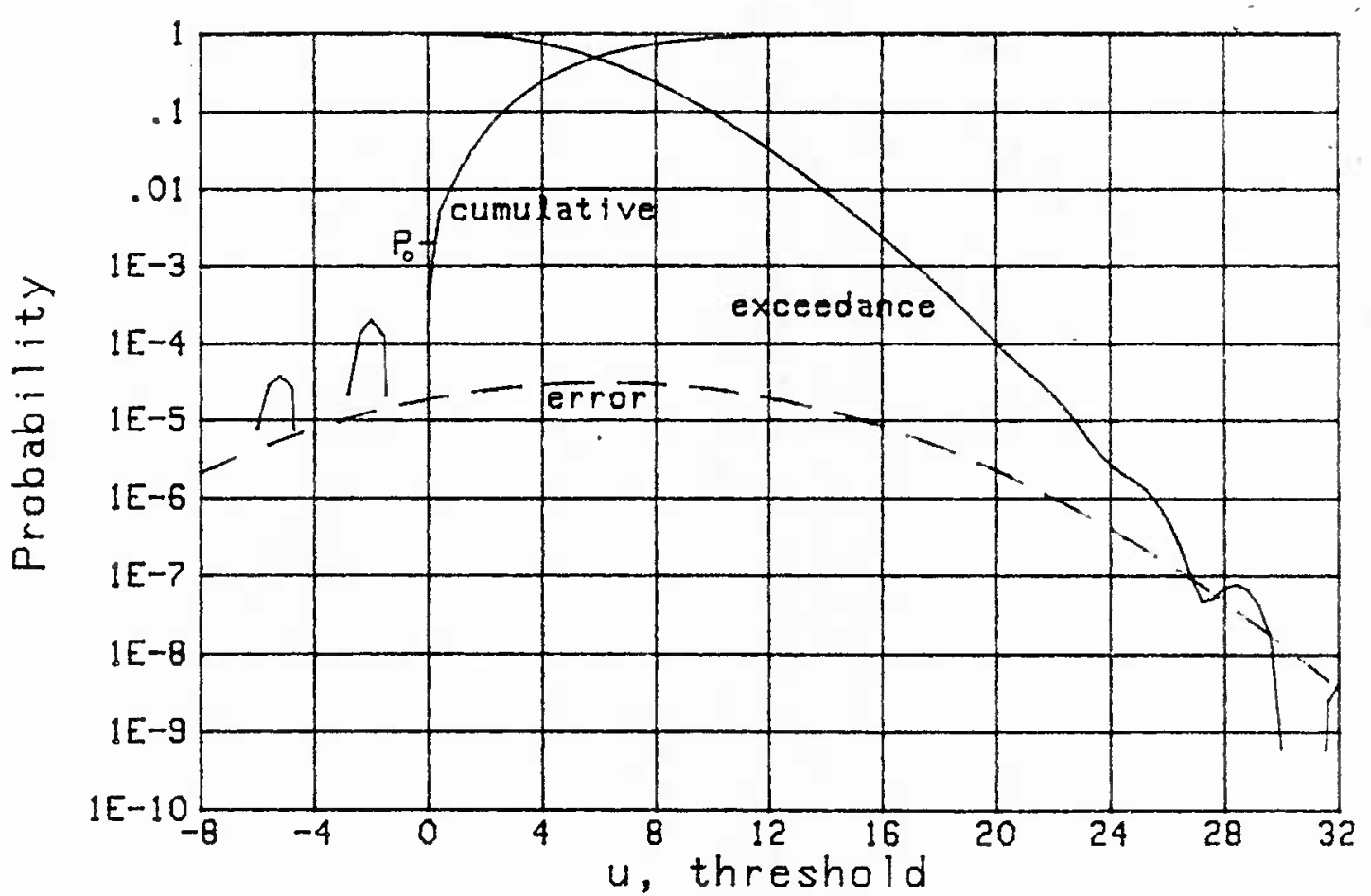

Figure 27. Estimated Error of Figure 8

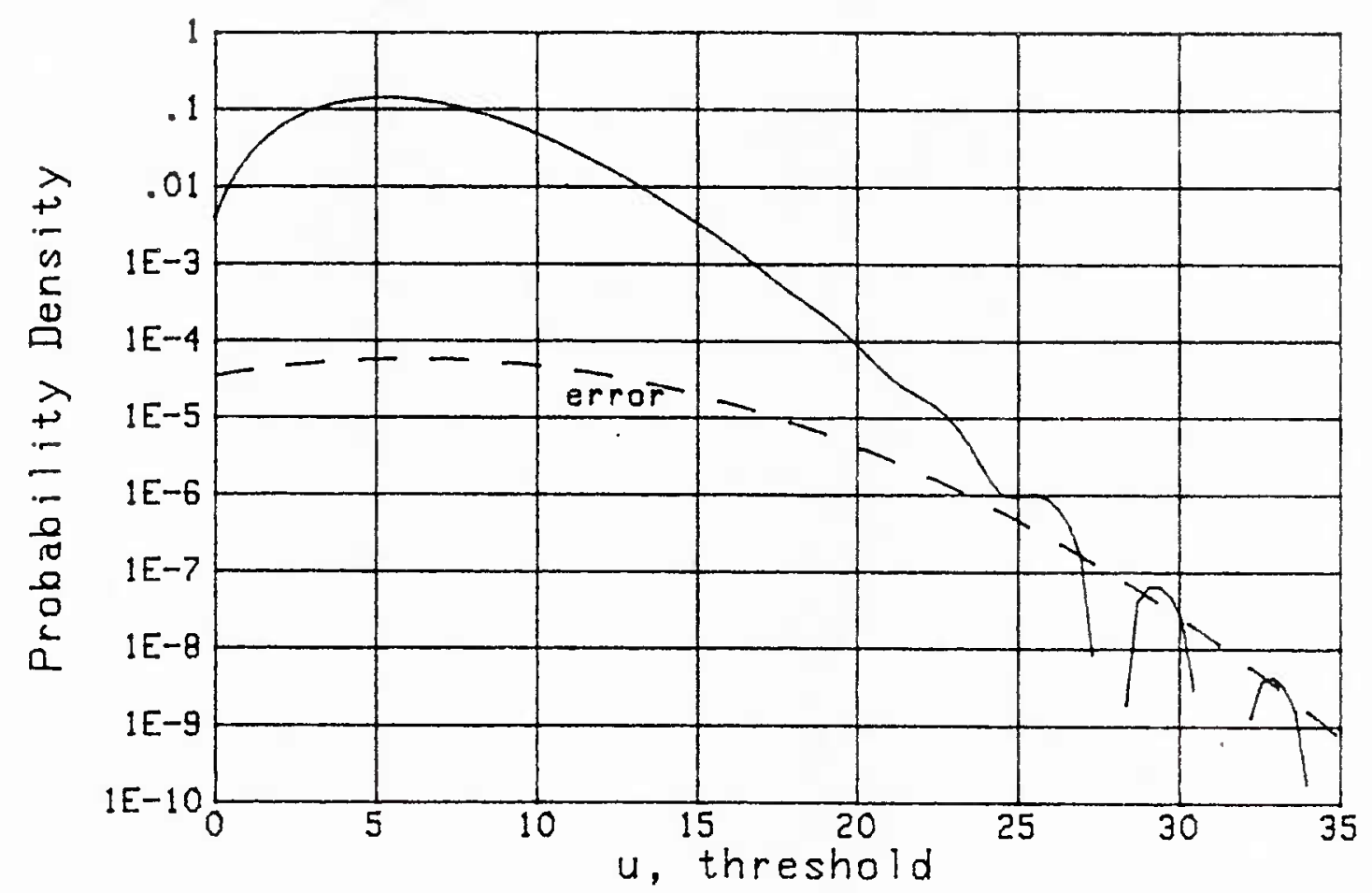

Figure 28. Estimated Error of Figure 10 
TR 7377

GENERAL IZED LAGUERRE EXPANSION

The details for the generalized Laguerre series are very similar to those above and so will be abbreviated. The envelope of the generalized Laguerre polynomial is $[7,8.22 .1]$

Env $\left\{L_{n}^{(\alpha)}(x)\right\} \sim \pi^{-\frac{1}{2}} e^{\frac{x}{2}} x^{-\frac{\alpha}{2}-\frac{1}{4}} n^{\frac{\alpha}{2}-\frac{1}{4}}$ as $n \rightarrow+\infty, \quad$ for $x>0$.

From (91) and (92), the $n$-th term of the approximate probability density.

function is

$$
\frac{u^{\alpha} \exp (-u / \beta)}{\beta^{\alpha+1} \Gamma\left(\alpha^{+1}\right)} b_{n}\left(\frac{n !}{\left(\alpha^{+}+\right)_{n}}\right)^{1 / 2} L_{n}^{(\alpha)}\left(\frac{u}{\beta}\right) .
$$

Then the magnitude of the error of the probability density function approximation is, for $u>0$,

$$
\begin{gathered}
E_{n}(u ; p) \equiv \frac{u^{\alpha} \exp (-u / \beta)}{\beta^{\alpha+1} \Gamma(\alpha+1)} \operatorname{Env}\left\{b_{n}\right\} \operatorname{Env}\left\{\left(\frac{n !}{(\alpha+1)_{n}}\right)^{1 / 2} L_{n}^{(\alpha)}\left(\frac{u}{\beta}\right)\right\}= \\
\sim\left[\pi \Gamma(\alpha+1) \beta^{2}\right]^{-1 / 2}\left(\frac{u}{\beta}\right)^{\frac{\alpha}{2}-\frac{1}{4}} \exp \left(-\frac{u}{2 \beta}\right) n^{-1 / 4} \operatorname{Env}\left\{b_{n}\right\} \text { as } n \rightarrow+\infty,
\end{gathered}
$$

where we used $[5,6.1 .47]$ and (181). This quantity peaks at $u=\beta\left(\alpha-\frac{1}{2}\right)$.

With regards to the cumulative distribution function, the $n$-th term of the approximation is, from (95) and (92), 


$$
\frac{u^{\alpha+1} \exp (-u / \beta)}{\beta^{\alpha^{+1}} \Gamma\left(\alpha^{+} 1\right)} b_{n} \frac{1}{n}\left(\frac{n !}{\left(\alpha^{+}+1\right)_{n}}\right)^{1 / 2} L_{n-1}^{(\alpha+1)}\left(\frac{u}{\beta}\right)
$$

Then the magnitude of the distribution error, for both the cumulative and the exceedance distribution functions, is roughly

$$
\begin{aligned}
& E_{n}(u ; P) \equiv \frac{u^{\alpha+1} \exp (-u / \beta)}{\beta^{\alpha+1} \Gamma(\alpha+1)} \operatorname{Env}\left\{b_{n}\right\} \operatorname{Env}\left\{\frac{1}{n}\left(\frac{n !}{(\alpha+1)_{n}}\right)^{1 / 2} L\left(\alpha_{n-1}^{(+1)}\left(\frac{u}{\beta}\right)\right\}=\right. \\
& \sim[\pi \Gamma(\alpha+1)]^{-\frac{1}{2}}\left(\frac{u}{\beta}\right)^{\frac{\alpha}{2}+\frac{1}{4}} \exp \left(-\frac{u}{2 \beta}\right) n^{-\frac{3}{4}} \text { Env }\left\{b_{n}\right\} \text { as } n \rightarrow+\infty \text {, for } u>0,
\end{aligned}
$$

upon use of (181). This quantity reaches its peak at $u=\beta\left(\alpha^{+} \frac{1}{2}\right)$.

An application of these results to the shot noise process, which was re-investigated in example I via the generalized Laguerre series, is given in figures 29 and 30 . Specifically, the approximate results from figures 20 and 22 have been repeated, and error measures (185) and (183), respectively, superposed as dashed lines. They confirm the earlier observations that the distribution and density approximations are reliable until the anomalous behavior on the tails manifests itself.

The difficult example $J$ is considered in figures 31 and 32 . Since the expansion coefficient sequence $\left\{b_{n}\right\}$ in figure 23 diverged for large $n$, the selection of $n=15$, as used in figures $24-26$, is not the large value needed to justify the use of (183) and (185). Thus, the dashed curves on figures 31 and 32 must be considered only as ball-park estimates; in general, the approximate error appears to be too conservative in these two figures. 


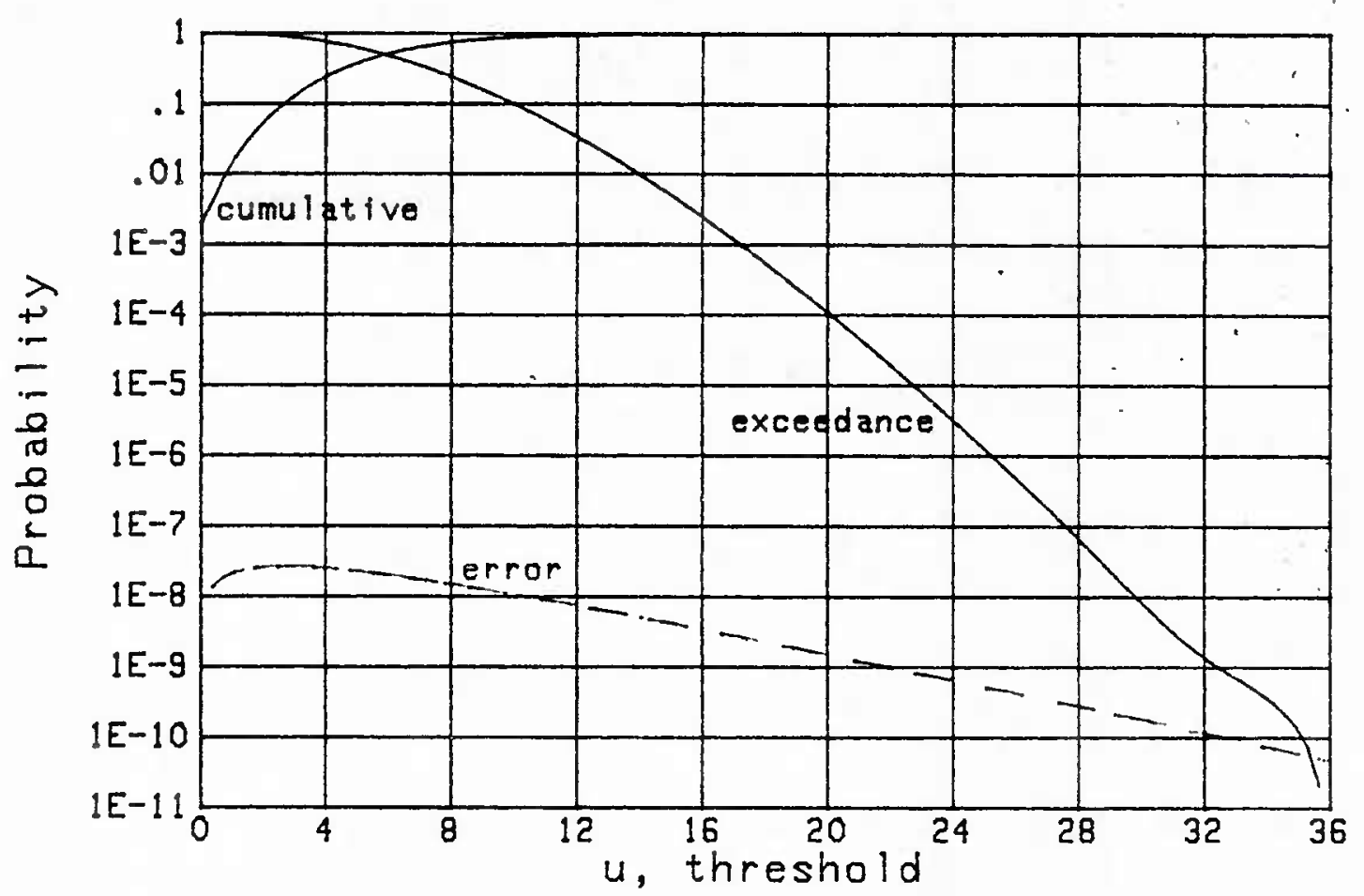

Figure 29. Estimated Error of Figure 20

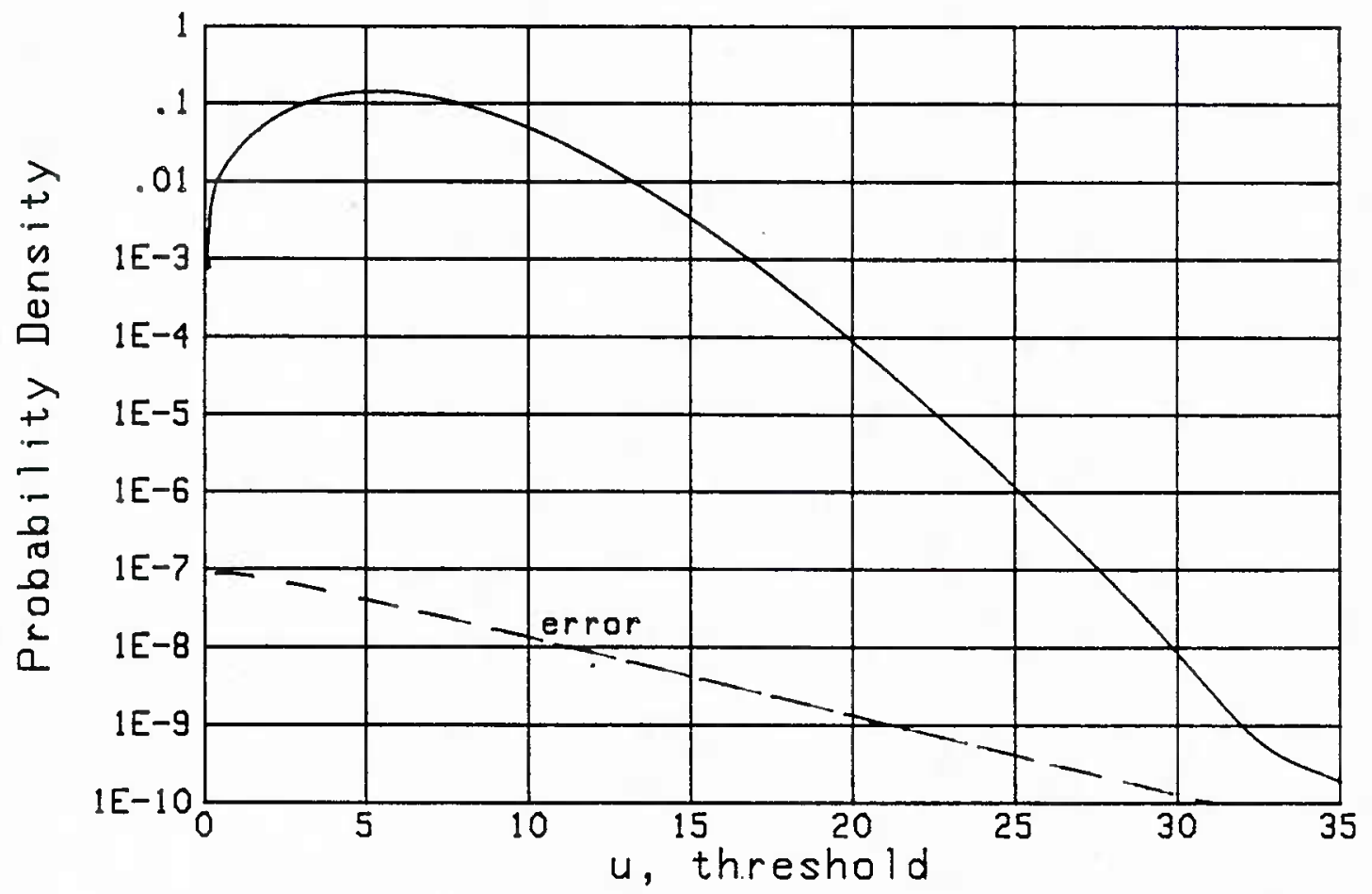

Figure 30. Estimated Error of Figure 22 


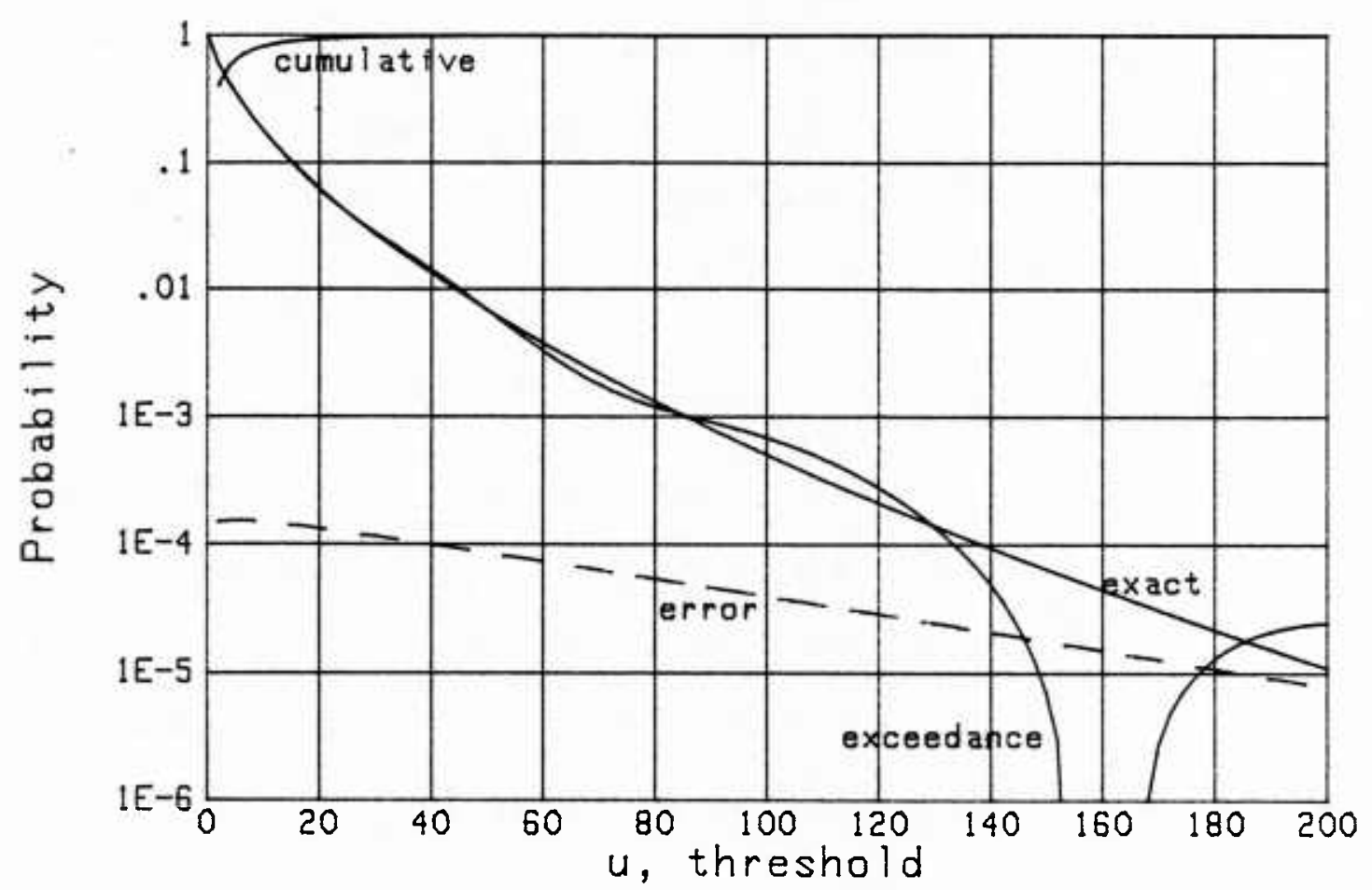

Figure 31. Estimated Error of Figure 24

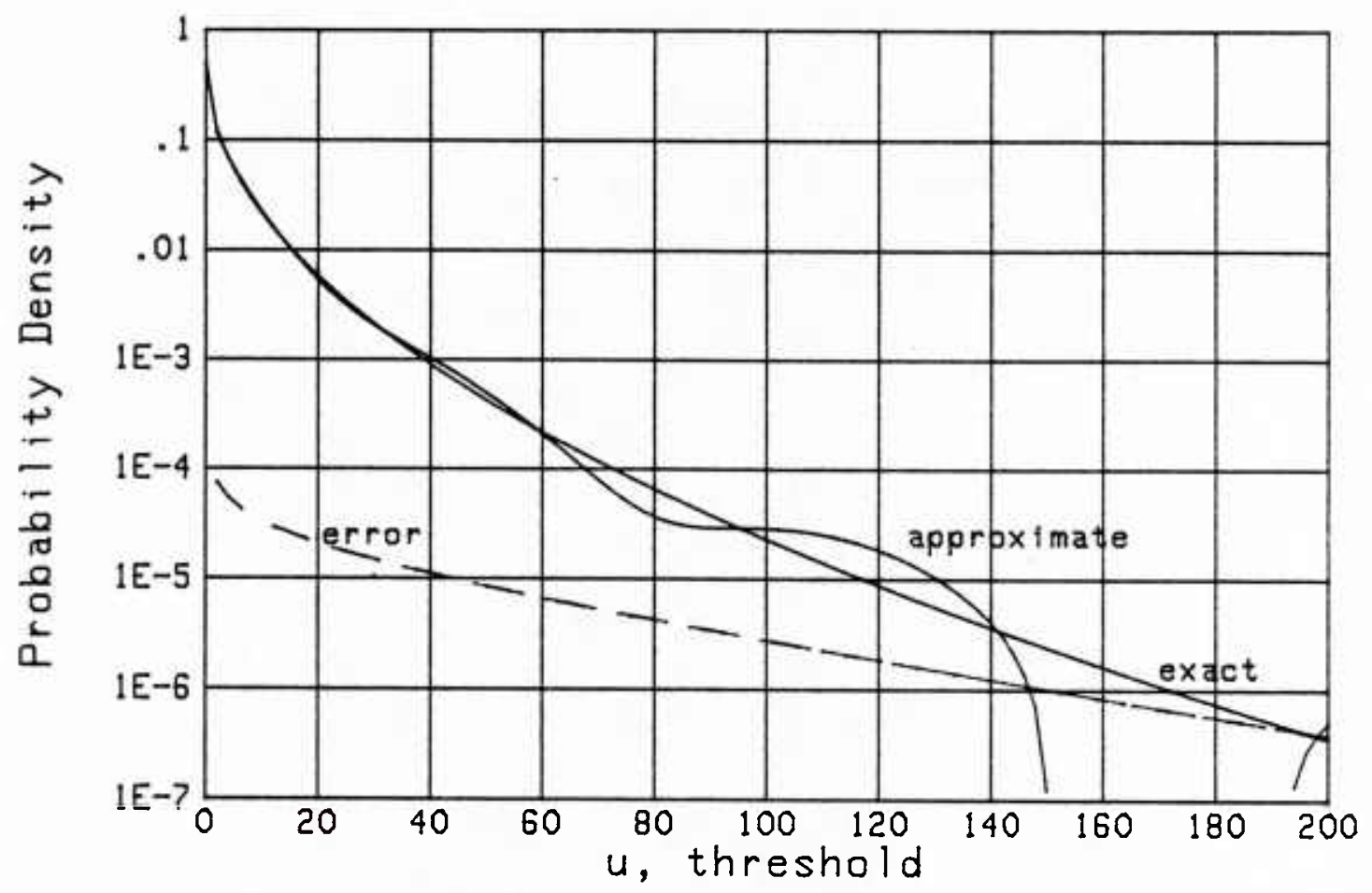

Figure 32. Estimated Error of Figure 26 
Finally, the Rice variate of example $G$ is re-considered in figure 33 . We took Env\{ $\left.b_{n}\right\} \approx 3 E-4$ at $n=65$, by extrapolating in figure 15 from smaller $n$, since round-off error is becoming significant by this value. It verifies the unreliability of the approximation in figure 33 for $u>7$.

Although all the examples in this report have the capability of evaluating either the moments or the cumulants via recursion, this is by no means necessary. Any method whatsoever of accurately calculating the starting statistics, be they moments or cumulants, is acceptable. For example, if a random variable with known probability density function $q$ is passed through a complicated nonlinearity $\mathrm{g}$, the moments of the output are given by

$$
\mu_{n}=\int d u g^{n}(u) q(u)
$$

These quantities could be evaluated for $0 \leq n \leq N$ by brute-force numerical procedures if necessary. The limit value $\mathrm{N}$ will depend on the accuracy with which $g$ and $q$ can be evaluated; if $g(u) \geq 0$ for all $u$, these integrals can be accomplished to a high degree of accuracy, thereby allowing large values of $\mathrm{N}$ to be employed. 


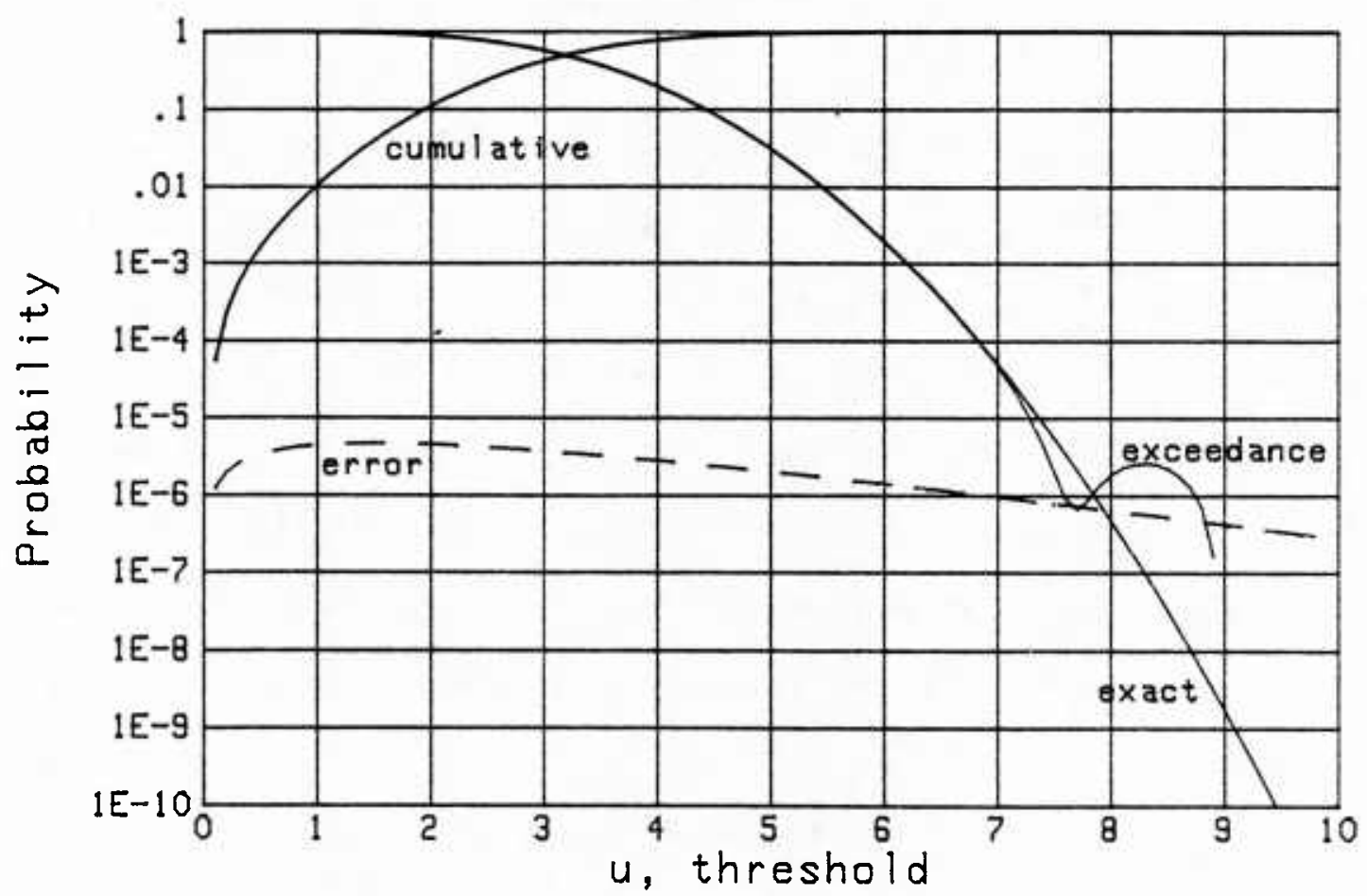

Figure 33. Estimated Error of Figure 16 
TR 7377

\section{DISCUSSION}

Several alternative methods have been presented for obtaining either Hermite or generalized Laguerre series expansions of probability density functions or cumulative and exceedance distribution functions, by means of recursive relations involving either moments or cumulants. Furthermore, estimates of the errors of the approximations are furnished so that the reliability can be assessed. Comparisons between approximations obtained by either the Hermite or the generalized Laguerre series afford an assessment of the accuracy of each; also, the availability of three alternative recursive procedures for the expansion coefficients allows for selection of the best method and results, and determination of the amount of round-off error.

The key feature to this approach is the rapid calculation and observation of the orthonormal expansion coefficients $\left\{b_{n}\right\}$ for each particular guess of weighting parameters $\alpha$ and $\beta$. A trial and error procedure is suggested for determining $\alpha$ and $\beta$ values that yield the set of fastest-decaying expansion coefficients. From observation of the expansion coefficients, the number of terms to retain in the series expansions is ascertained, being sure to avoid the effects of round-off error which dominates the calculated expansion coefficients $\left\{b_{n}\right\}$ for large $n$. Since the amount and location of round-off error on the plot of expansion coefficients also depends on $\alpha$ and $B, a$ judicious search may be required to find acceptable weighting parameter values. Of course, a computer with a larger number of significant digits would greatly alleviate this drawback; the particular computer used for all the calculations reported here is the Hewlett-Packard 9000 Model 520 which 
devotes 52 bits (15.65 decimal digits) to the mantissa and 11 bits to the exponent. Failure of the technique is indicated by divergence of the expansion coefficient sequence $\left\{b_{n}\right\}$.

Programs for the shot noise process considered in examples $D$ and $I$ are presented in appendix F. Times of execution are as follows. For the Hermite series, the 80 cumulants or 80 moments required as input for figure 7 took .7 or .35 seconds, respectively. The calculation, plotting, and display of the 80 expansion coefficients in figure 7 took 1.6 seconds via the RC approach and 1.75 seconds via the two moment approaches. The computation and display of the 100-point plots of the cumulative distribution function in figure 8 and the probability density function in figure 9, each using 65 terms in the series expansion, took 1.1 and .95 seconds, respectively.

For the generalized Laguerre series, the 70 cumulants or 70 moments required as input for figure 19 took .54 seconds or .28 seconds, respectively. The calculation and display of the 70 expansion coefficients in figure 19 took 1.8 seconds via the RC approach and 1.5 seconds via the two moment approaches. The computation and display of the 100-point plots of the cumulative distribution function in figure 20 and the probability density function in figure 21 took 1.1 and .7 seconds, respectively. These execution times are short enough to allow a human observer to conduct a rapid trial-and-error search of $\alpha, \beta$ space, determine adequate parameter values, and assess their accuracy.

Alternative exact procedures for determination of cumulative and exceedance distribution functions via characteristic functions have been presented in $[9,10,11]$. Those methods generally have the potential for 
TR 7377

greater accuracy, are less subject to round-off-error, and would be preferred if possible. However, analysis of systems with nonlinearities and memory sometimes precludes or greatly hinders their application; in such cases, the current approach is a very good candidate for consideration.

The two weightings in (1) and (2), namely the Hermite and generalized Laguerre, have been investigated rather intensively here, because so many properties and recursions are available for the corresponding (orthonormal) polynomials. These properties have been utilized to derive simple recursive relations for the expansion coefficients and density and distribution functions, thereby realizing quick efficient procedures for numerical evaluation and observation.

It would be extremely useful to be able to extend these resuits to the weighting

$$
u^{\alpha} \exp \left(-u^{2} / \beta^{2}\right) \text { for } u>0 \text {, }
$$

since this class of probability density functions is often encountered in nonlinear systems with Gaussian inputs. However, there are several pivotal recursive relations for the corresponding orthonormal polynomials that would be needed, and it is questionable if a fast procedure could be devised without them. Also, it is unknown if recursive procedures for the expansion coefficients in terms of moments or cumulants could be derived, as was done here for the Hermite and generalized Laguerre weightings. This is a topic worthy of further investigation. 
APPENDIX A. COEFFICIENT RECURSION FOR EXPONENTIAL OF POWER SERIES

Suppose power series $\sum_{n=0}^{\infty} h_{n} z^{n}$ converges for some $|z|>0$, and we exponentiate it, getting a new power series

$$
\sum_{n=0}^{\infty} g_{n} z^{n}=\exp \left\{\sum_{n=0}^{\infty} n_{n} z^{n}\right\} \text {. }
$$

Then the lowest order coefficient is

$$
g_{0}=\exp \left(h_{0}\right)
$$

while for $k \geq 1$, we have

$$
\begin{aligned}
& g_{k}=\frac{1}{k !}\left(\frac{d}{d z}\right)^{k}\left[\exp \left\{\sum_{n=0}^{\infty} n_{n} z^{n}\right\}\right]_{z=0}= \\
& =\frac{1}{k !}\left(\frac{d}{d z}\right)^{k-1}\left[\sum_{n=1}^{\infty} n h_{n} z^{n-1} \exp \left\{\sum_{n=0}^{\infty} n_{n} z^{n}\right\}\right]_{z=0}= \\
& =\frac{1}{k !} \sum_{p=0}^{k-1}\left(\begin{array}{c}
k-1 \\
p
\end{array}\right)\left(\frac{d}{d z}\right)^{p}\left[\sum_{n=1}^{\infty} n n_{n} z^{n-1}\right]_{z=0}\left(\frac{d}{d z}\right)^{k-1-p}\left[\exp \left\{\sum_{n=0}^{\infty} n_{n} z^{n}\right\}\right]_{z=0}= \\
& =\frac{1}{k !} \sum_{p=0}^{k-1}\left(\begin{array}{c}
k-1 \\
p
\end{array}\right)(p+1) ! h_{p+1}(k-1-p) ! g_{k-1-p}= \\
& =\frac{1}{k} \sum_{p=0}^{k-1}(p+1) h_{p+1} g_{k-1-p}=\frac{1}{k} \sum_{m=1}^{k} m n_{m} g_{k-m} .
\end{aligned}
$$

Thus we have the recursion for coefficients $\left\{g_{k}\right\}$ in terms of the $\left\{h_{m}\right\}$ :

$$
g_{k}=\frac{1}{k} \sum_{m=1}^{k} m h_{m} g_{k-m} \quad \text { for } k \geq 1, \quad g_{0}=\exp \left(h_{0}\right) \text {. }
$$


TR 7377

If we now refer to (6) and (7) and identify

$$
g_{n}=\mu_{n} / n !, \quad h_{n}=x_{n} / n !
$$

there follows the moments in terms of the cumulants according to

$$
u_{k}=\sum_{m=0}^{k-1}\left(\begin{array}{c}
k-1 \\
m
\end{array}\right) X_{k-m} \mu_{m} \quad \text { for } k \geq 1, \quad \mu_{0}=\exp \left(X_{0}\right)
$$

This is a slight generalization of $[6,(10)]$. This equation is immediately inverted, to yield the cumulants in terms of moments:

$$
\chi_{k}=\frac{1}{\mu_{0}}\left[\mu_{k}-\sum_{m=1}^{k-1}\left(\begin{array}{c}
k-1 \\
m
\end{array}\right) \chi_{k-m} \mu_{m}\right] \text { for } k \geq 1, \quad \chi_{0}=\ln \mu_{0},
$$

which generalizes $[6,(11)]$.

In terms of the normalized cumulants and moments defined in (62) and (69) respectively, we have

$$
\hat{u}_{k}=\frac{1}{k} \sum_{m=0}^{k-1} \hat{\chi}_{k-m} \hat{\mu}_{m} \quad \text { for } k \geq 1, \quad \hat{\mu}_{0}=\exp \left(\chi_{0}\right),
$$

and

$$
\hat{\chi}_{k}=\frac{1}{\hat{\mu}_{0}}\left[k \hat{\mu}_{k}-\sum_{m=1}^{k-1} \hat{\chi}_{k-m} \hat{\mu}_{m}\right] \text { for } k \geq 1,\left(\chi_{0}=\ln \mu_{0}\right) \text {. }
$$




\section{APPENDIX B. EXPANSION OF $\mathrm{He}_{n}(x+y)$}

The quantity $\mathrm{He}_{n}(x+y)$ is a polynomial of degree $n$ in $y$. Therefore we can expand

$$
H_{n}(x+y)=\sum_{m=0}^{n} \gamma_{m} \frac{y^{m}}{m !},
$$

where $\gamma_{m}$ will also depend on $n$ and $x$. In fact,

$$
\begin{aligned}
& r_{m}=\left(\frac{\partial}{\partial y}\right)^{m}\left[H e_{n}(x+y)\right]_{y=0}=\left(\frac{\partial}{\partial t}\right)^{m}\left[H_{n}(t)\right]_{t=x}= \\
& =\left(\frac{\partial}{\partial t}\right)^{m-1}\left[n H_{n-1}(t)\right]_{t=x}=n(n-1) \ldots(n-m+1) H e_{n-m}(x)= \\
& =\frac{n !}{(n-m) !} H e_{n-m}(x),
\end{aligned}
$$

where we used $[5,22.8 .8]$ repeatedly. Using $(B-2)$ in $(B-1)$, we have the alternative forms for the expansion,

$$
\begin{aligned}
H e_{n}(x+y) & =\sum_{m=0}^{n}\left(\begin{array}{l}
n \\
m
\end{array}\right) H e_{n-m}(x) y^{m}= \\
& =\sum_{m=0}^{n}\left(\begin{array}{l}
n \\
m
\end{array}\right) H e_{n-m}(y) x^{m}= \\
& =\sum_{k=0}^{n}\left(\begin{array}{l}
n \\
k
\end{array}\right) H e_{k}(y) x^{n-k} \text { for } n \geq 0 .
\end{aligned}
$$


We have, from (94),

$$
I_{n}(y)=\int_{0}^{y} d x x^{\alpha} e^{-x} L_{n}^{(\alpha)}(x) \text { for } n \geq 0 .
$$

Then

$$
I_{0}(y)=\int_{0}^{y} d x x^{\alpha} e^{-x} 1=\gamma(\alpha+1, y)=\frac{y^{\alpha+1} e^{-y}}{\alpha^{+1}}{ }_{1} F_{1}(1 ; \alpha+2 ; y),
$$

using $[5,22.4 .7,6.5 .2$, and 6.5.12]. Also, we have from $[5,22.11 .6]$,

$$
x^{\alpha} e^{-x} L_{n}^{(\alpha)}(x)=\frac{1}{n !}\left(\frac{d}{d x}\right)^{n}\left\{e^{-x} x^{\alpha^{+} n}\right\} .
$$

Then for $n \geq 1,(C-1)$ can be developed as

$$
\begin{aligned}
& I_{n}(y)=\int_{0}^{y} d x \frac{1}{n !}\left(\frac{d}{d x}\right)^{n}\left\{e^{-x} x^{\alpha+n}\right\}= \\
& =\frac{1}{n !} \int_{0}^{y} d\left(\frac{d}{d x}\right)^{n-1}\left\{e^{-x} x^{\alpha+n}\right\}=\frac{1}{n !}\left(\frac{d}{d y}\right)^{n-1}\left\{e^{-y} y^{\alpha+n}\right\}= \\
& \quad=\frac{1}{n} y^{\alpha+1} e^{-y} L_{n-1}^{\left(\alpha^{+} 1\right)}(y),
\end{aligned}
$$

where we set the lower limit of the evaluated integral to zero since $\alpha^{+n} \geq \alpha^{+1}>0$. 
APPENDIX D. FOURIER TRANSFORM OF GENERAL IZED LAGUERRE POLYNOMIAL

We wish to evaluate transform

$$
A(\omega)=\int_{0}^{\infty} d t e^{i \omega t} t^{\alpha} e^{-t} L_{n}^{(\alpha)}(t) .
$$

Now

$$
n ! t^{\alpha} e^{-t} L_{n}^{(\alpha)}(t)=\left(\frac{d}{d t}\right)^{n}\left\{e^{-t} t^{\alpha+n}\right\} \text { for } n \geq 0,
$$

according to $[5,22.11 .6]$. Therefore for $n \geq 1$,

$$
\begin{aligned}
n ! A(\omega) & =\int_{0}^{\infty} d t e^{i \omega t}\left(\frac{d}{d t}\right)^{n}\left\{e^{-t} t^{\alpha+n}\right\}= \\
& =\int_{0}^{\infty} e^{i \omega t} d\left(\frac{d}{d t}\right)^{n-1}\left\{e^{-t} t^{\alpha+n}\right\}= \\
& =-i \omega \int_{0}^{\infty} d t e^{i \omega t}\left(\frac{d}{d t}\right)^{n-1}\left\{e^{-t} t^{\alpha+n}\right\},
\end{aligned}
$$

where we used integration by parts with the fact that the integrated part is zero at $t=0$ and $\infty$, since $\alpha^{+n} \geq \alpha^{+1}>0$. Repeated integration by parts then yields

$$
n ! A(\omega)=(-i \omega)^{n} \int_{0}^{\infty} d t e^{i \omega t} e^{-t} t^{\alpha^{+n}}=\Gamma\left(\alpha^{+1+n}\right) \frac{(-i \omega)^{n}}{(1-i \omega)^{\alpha+1+n}} .
$$

This is the result quoted in (104). 
The starting point is the moment expression in (142):

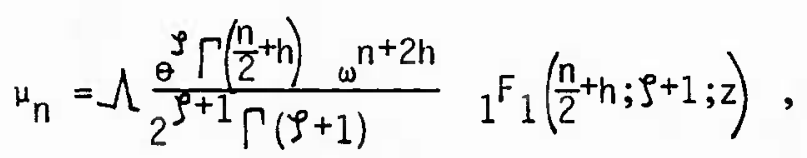

where $h=(\gamma+\rho+1) / 2, z=\omega^{2} \theta^{2} / 4$. Denote the ${ }_{1} F_{1}$ term in $(E-1)$ by $F_{n}$, and the leading factor by $G_{n}$; thus $\mu_{n}=G_{n} F_{n}$. There follows immediately

$$
G_{n}=G_{n-2} \omega^{2}\left(\frac{n}{2}+n-1\right) \quad \text { for } n \geq 2
$$

For the ${ }_{1} F_{1}$ function, we refer to $[5,13.4 .1]$ to get

$$
F_{n}=\frac{1}{\frac{n}{2}+h-1}\left[(n+2 h-3-\rho+z) F_{n-2}+\left(\rho+2-\frac{n}{2}-h\right) F_{n-4}\right] \text {. }
$$

If we substitute $(E-2)$ and $(E-3)$ into $\mu_{n}=G_{n} F_{n}$, and then re-apply $(E-2)$ in the second term, we obtain

$$
\mu_{n}=\omega^{2}(n+\gamma-2+z) \mu_{n-2}-\frac{1}{4} \omega^{4}\left[(n+\gamma-3)^{2}-\rho^{2}\right] \mu_{n-4} ;
$$

we also eliminated $h$. Starting values for $\mu_{n}$ can be obtained from (E-1).

For the special case (143) and (144), (E-4) reduces to

$$
\mu_{n}=\omega^{2}(n-1+z) \mu_{n-2}-\frac{1}{4} \omega^{4}(n-2)^{2} \mu_{n-4},
$$


TR 7377

with starting values

$$
\begin{aligned}
& \mu_{0}=1, \quad \mu_{1}=\frac{1}{2} \pi^{1 / 2} \omega e^{-z} F_{1}\left(\frac{3}{2} ; 1 ; z\right), \\
& \mu_{2}=\omega^{2}(1+z), \quad \mu_{3}=\frac{3}{4} \pi^{1 / 2} \omega^{3} e^{-z} F_{1}\left(\frac{5}{2} ; 1 ; z\right) .
\end{aligned}
$$

Kummer's transformation $[5,13.1 .27]$ was employed in this last equation; these forms afford accurate starting values for recursion (E-5). 


\section{APPENDIX F. PROGRAM LISTINGS}

Eight programs are listed in this appendix. They are given in BASIC for the Hewlett Packard 9000 Model 520 computer. For ease of reference, a shorthand notation is adopted:

$\begin{array}{lll}P & \text { denotes } & \text { cumulative or exceedance distribution function } \\ \text { P } & \text { denotes } & \text { probability density function } \\ H & \text { denotes } & \text { Hermite expansion } \\ \text { L } & \text { denotes } & \text { generalized Laguerre expansion } \\ \text { RC } & \text { denotes } & \text { recursively via cumulants } \\ \text { DM } & \text { denotes } & \text { directly via moments } \\ \text { RM } & \text { denotes } & \text { recursively via moments }\end{array}$

Table F-1. Shorthand Notation

Then, for example, the combination PHRC means that this program yields the cumulative or exceedance distribution function in terms of a Hermite expansion, by means of expansion coefficients determined recursively via cumulants. The eight programs listed here are, in order, 
TR 7377

$\begin{array}{ll}\text { PHRC } & \text { Figures } 7 \text { and } 8 \\ \text { pHRC } & \text { Figures } 7,9 \text {, and } 10 \\ \text { PHDMandRM } & \text { Figures } 7 \text { (and } 8 \text { ) } \\ \text { pHDMandRM } & \text { Figures } 7 \text { (and 9, 10) } \\ \text { PLRC } & \text { Figures } 19 \text { and } 20 \\ \text { PLRC } & \text { Figures } 19,21 \text {, and 22 } \\ \text { PLDMandRM } & \text { Figures } 19 \text { (and 20) } \\ \text { pLDMandRM } & \text { Figures } 19 \text { (and 21, 22) }\end{array}$

Table F-2. Program Abbreviations

The combination DMandRM means that this program gives the expansion coefficients directly via moments as well as recursively via moments; the user must select the procedure of interest.

The only input statistics we have given a listing for here is the shot noise process used in examples $D$ and $I$; in particular, the cumulant and moment routines are listed at the very end of PHRC and PHDMandRM, respectively. The figure references given in table F-2 indicate where each particular program was used in this report; the parenthetical references are alternative ways of generating those figures. The remaining figures in this report require that the cumulant and moment subroutines be replaced by the appropriate statistics of interest. 
To save space, no subroutines are listed more than once; instead, comments are made indicating where the needed routines are located, according to the coding in table F-2. For example, in program PHDMandRM, function subprogram FNPhi, Tine 570 , the comment is made that this routine has already been listed in PHRC.

We now explain some of the details of the PHRC program, as an example, so that a user can apply these techniques and routines to his particular problem. The user must specify $M$ in 1 ine 30 , which is the maximum order of approximation desired, or the number of cumulants or moments that can be calculated. The notation DOUBLE in 1 ine 40 denotes INTEGER variables. The user must select $\alpha$ and $\beta$ in lines 130,140; if they are chosen equal to $\alpha_{0}, \beta_{0}$ which have been computed in 1 ines 110,120 , then expansion coefficients $a_{1}=a_{2}=0$, or equivalently $b_{1}=b_{2}=0$. However, this choice is recommended onty as a starter on the search in $\alpha, \beta$ space.

The CALL in line 150 is to the subroutine which calculates the expansion coefficients for a Hermite series, recursively via cumulants, as can be deciphered from the abbreviated subroutine title. The expansion coefficients $\left\{b_{n}\right\}$ are calculated and the running sum of $b_{n}^{2}$ is calculated, both of which are printed on the CRT vs $n$. Also, a plot of the expansion coefficients $\left\{b_{n}\right\}$ is made in this subroutine, from which the user must decide on the order, $N$, to employ in the approximate cumulative and exceedance distribution function; alternatively, he can reject the sequence of $\left\{b_{n}\right\}$ so obtained, and re-run the program with different $\alpha, \beta$ values. 
TR 7377

When a satisfactory $\alpha, \beta$ pair is obtained, the limits $u_{1}, u_{2}$ on the range of arguments of the distribution must also be specified; this selection is aided by the print-out of the center and rms width of the density under investigation. A plot of 100 values of the cumulative and exceedance distribution functions is then made on a logarithmic ordinate. The various subroutines are self-explanatory and are keyed to the equation numbers in this report. 


\section{PROGRAM PHRC}

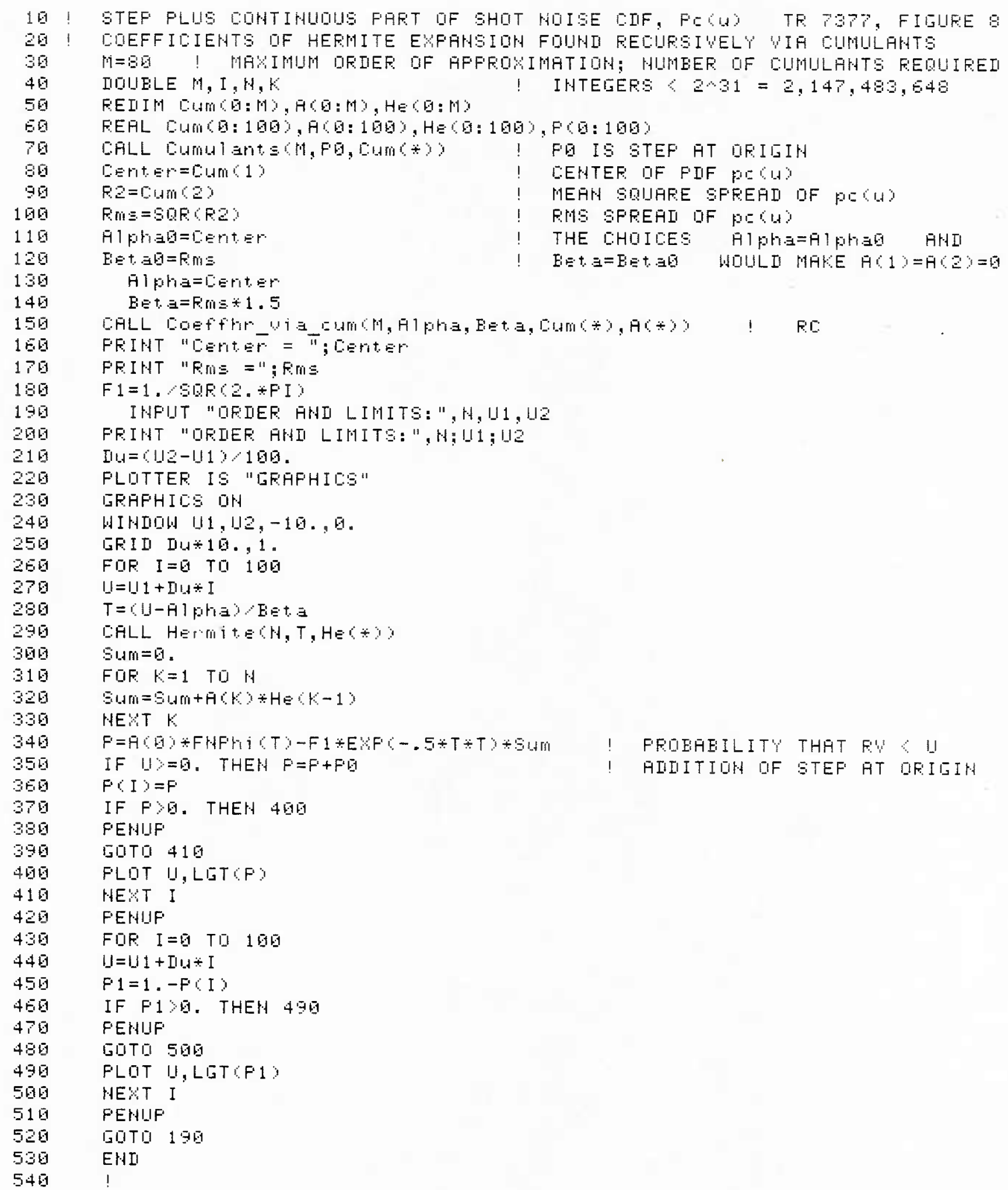




\section{PROGRAM PHRC (cont'd)}

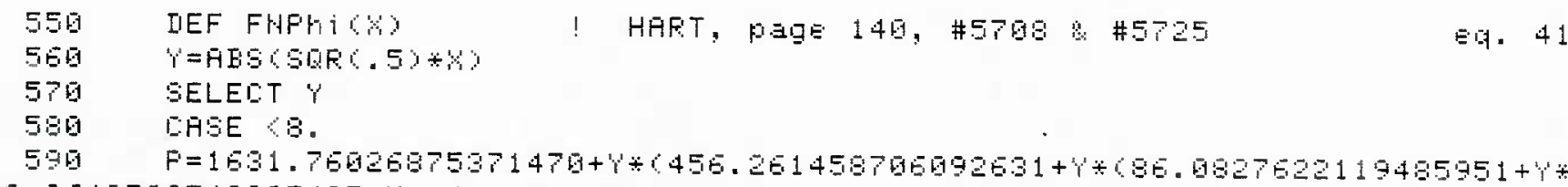
(10. $9649599749695425+\% \div .564189586761913614)$

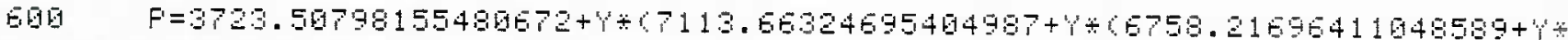
$4632.26701683094974+Y \div \% 3$

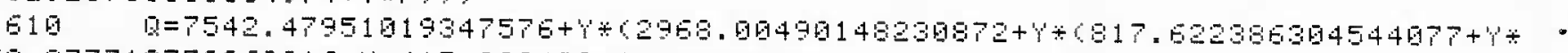
(5) $157710750362216+1 \div(1.8394984391395565+4) 3)$

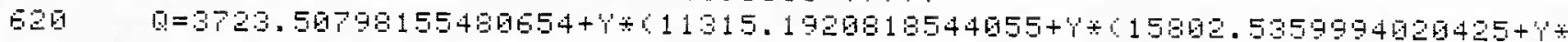
$61349.946561284457+\gamma \div 9) ?$

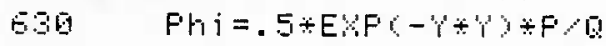

546 CHEE $\angle 2 E .5$

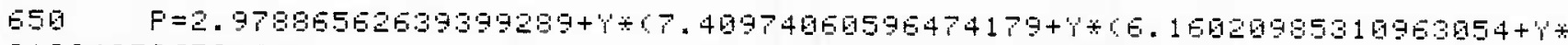

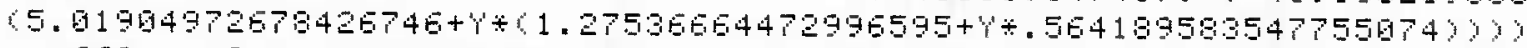

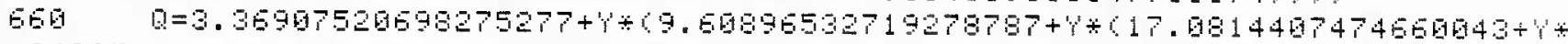

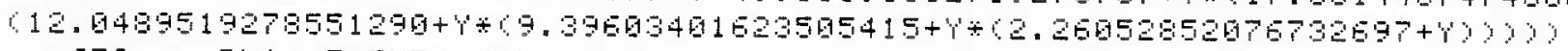

ET

ESG LASE ELSE

E96 Fhi

700 EHII SELEIT

710 IF $\mathrm{Y}>\mathrm{G}$. THEH Fhi=1. $\mathrm{FHi}$

72 FETUFH FHi

T3G FHEHI

740 !

$7 E$ TIUELE

7 P

$780 \quad H E 13=\%$

790 FOF $k=2$ TO H

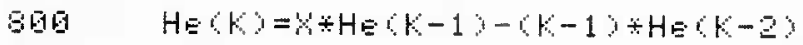

816 HEXT

B2G SUEEHI

日30 !

846

850

800

870

880

896

960

916

920

950

940

950

956

970

SUlE Plom [UIUELE K.J

FEAL MOMG

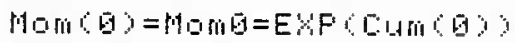

FDR $K=1$ TO M

$T=1$.

$S=C A m C K J \div W G$

FOR $I=1$ TU $k-1$

$T=T \div C \mathrm{~K}-\mathrm{J} \geqslant \mathrm{J}$

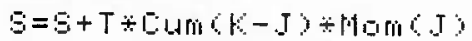

HEXT I

Mon $603=8$

HEST $K$

980

SIIBEHII 
PROGRAM PHRC (cont'd)

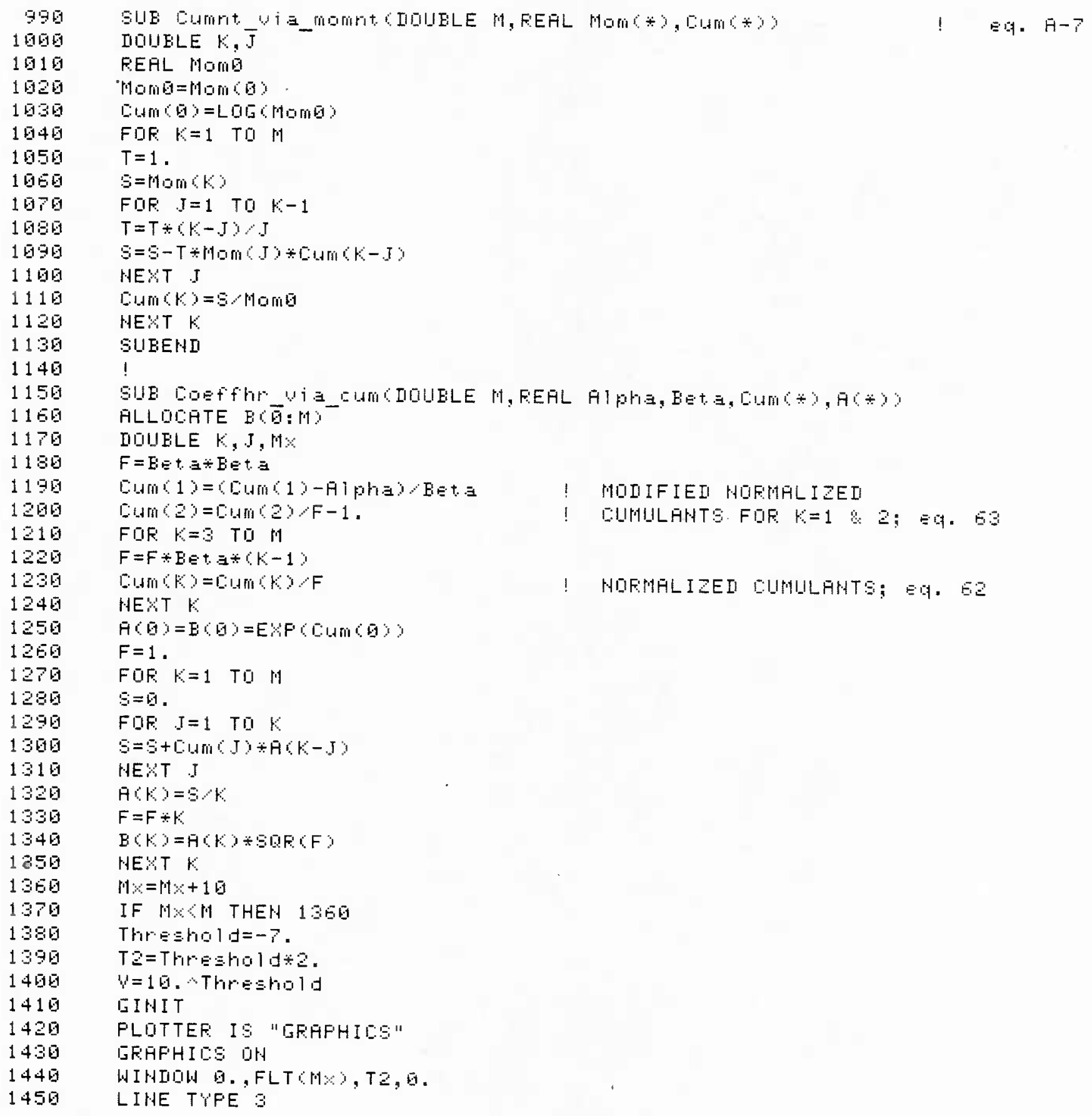




\section{PROGRAM PHRC (cont'd)}

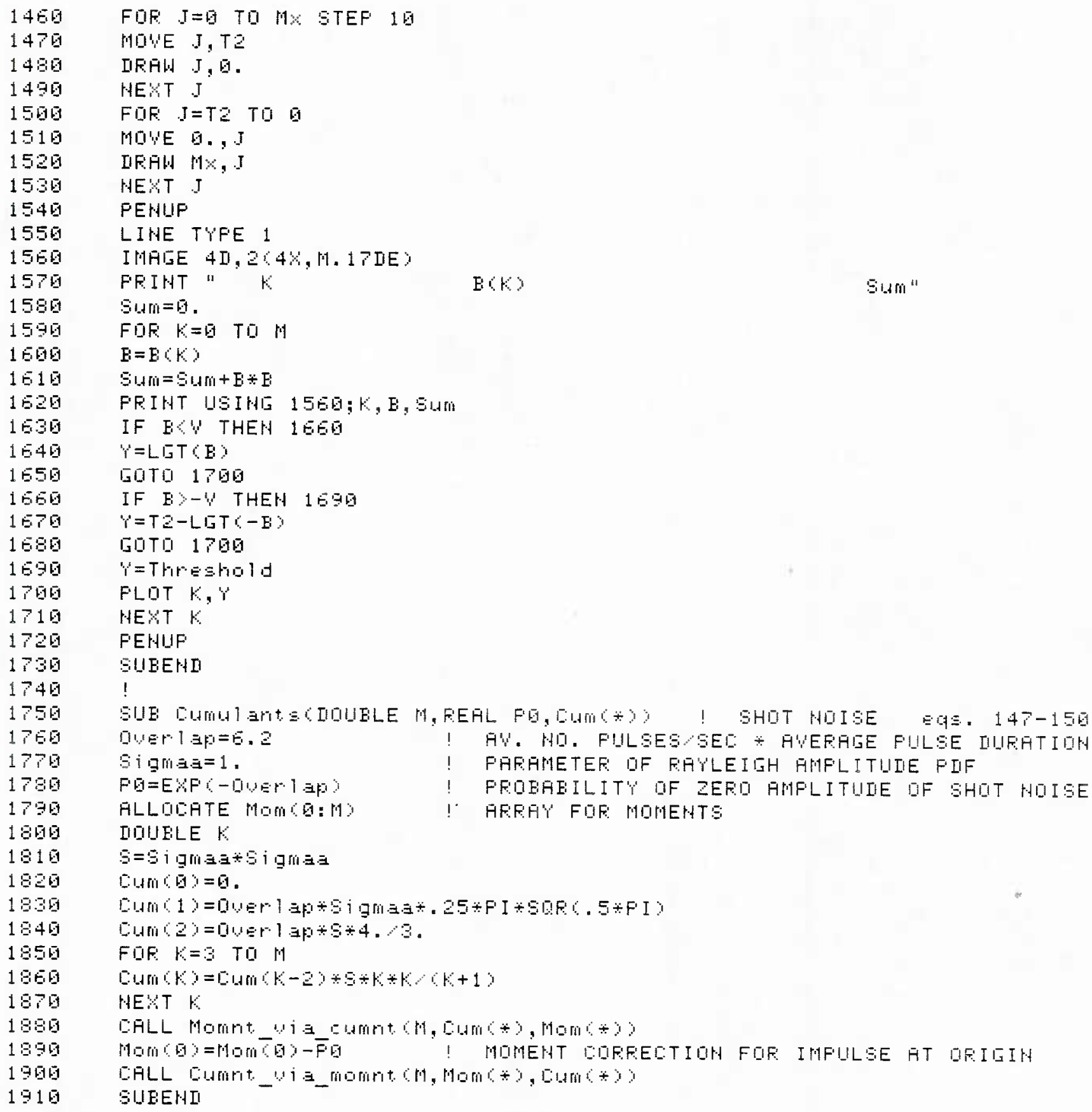




\section{PROGRAM PHRC}

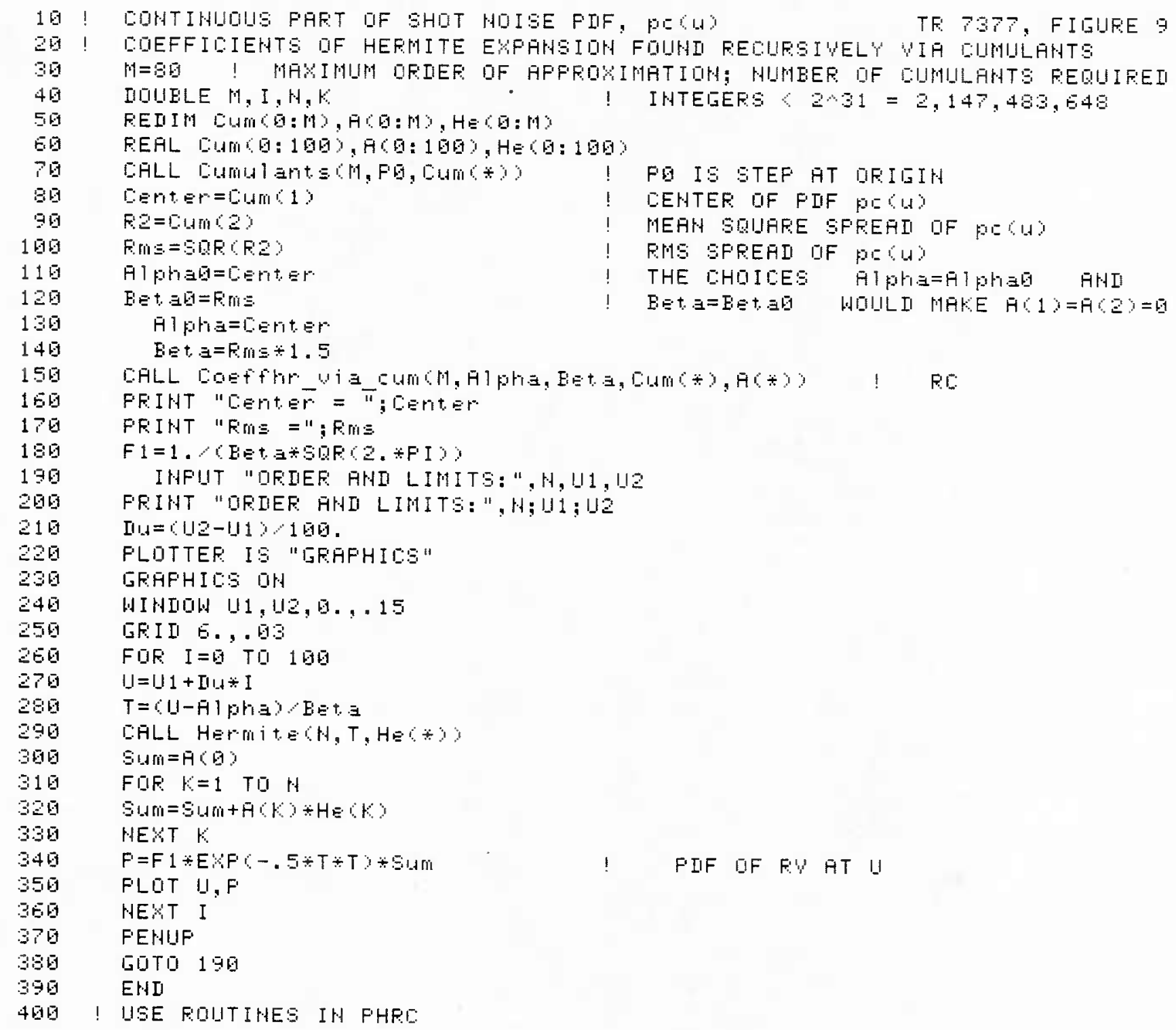




\section{PROGRAM PHDMandRM}

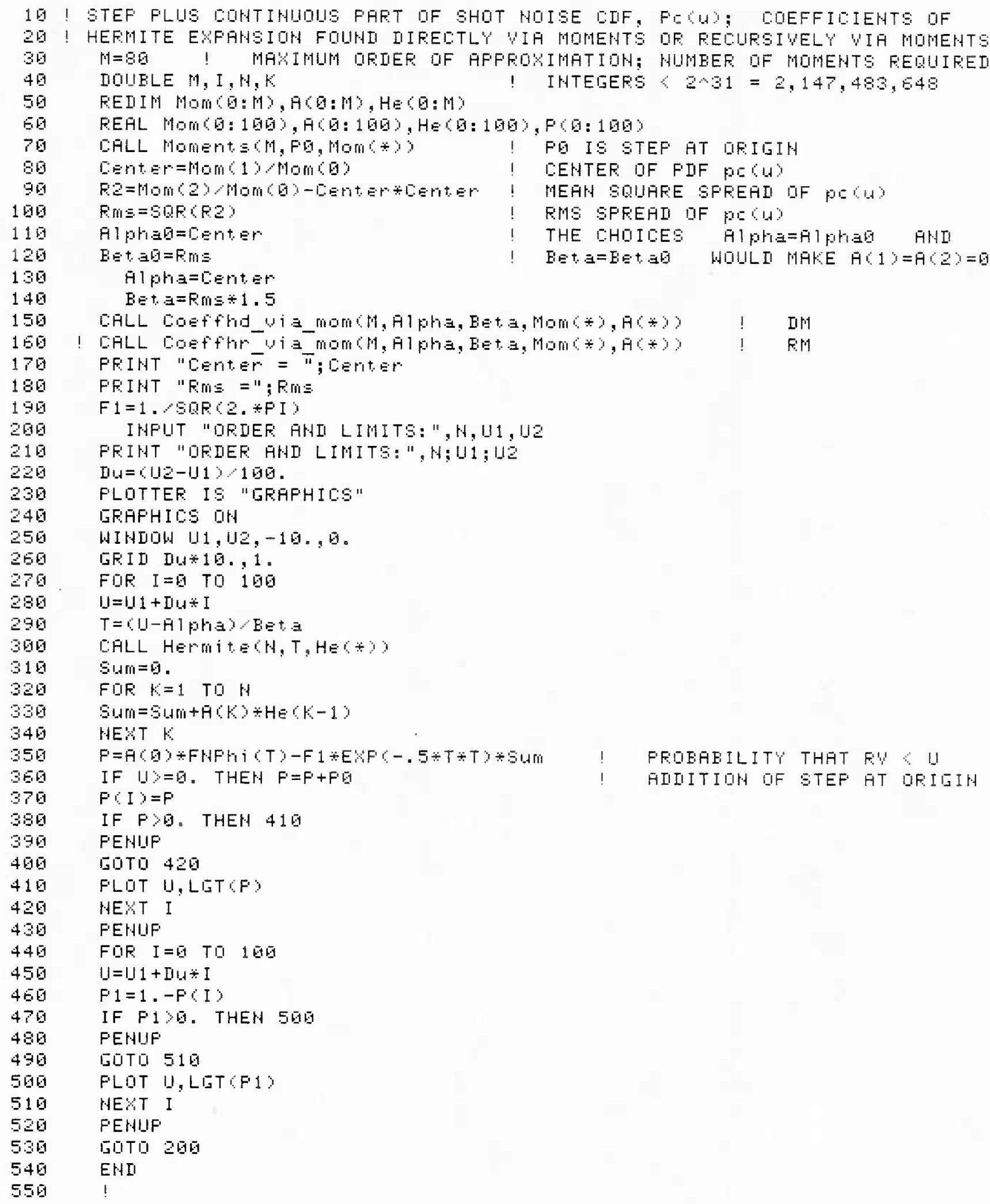




\section{PROGRAM PHDMandRM (cont'd)}

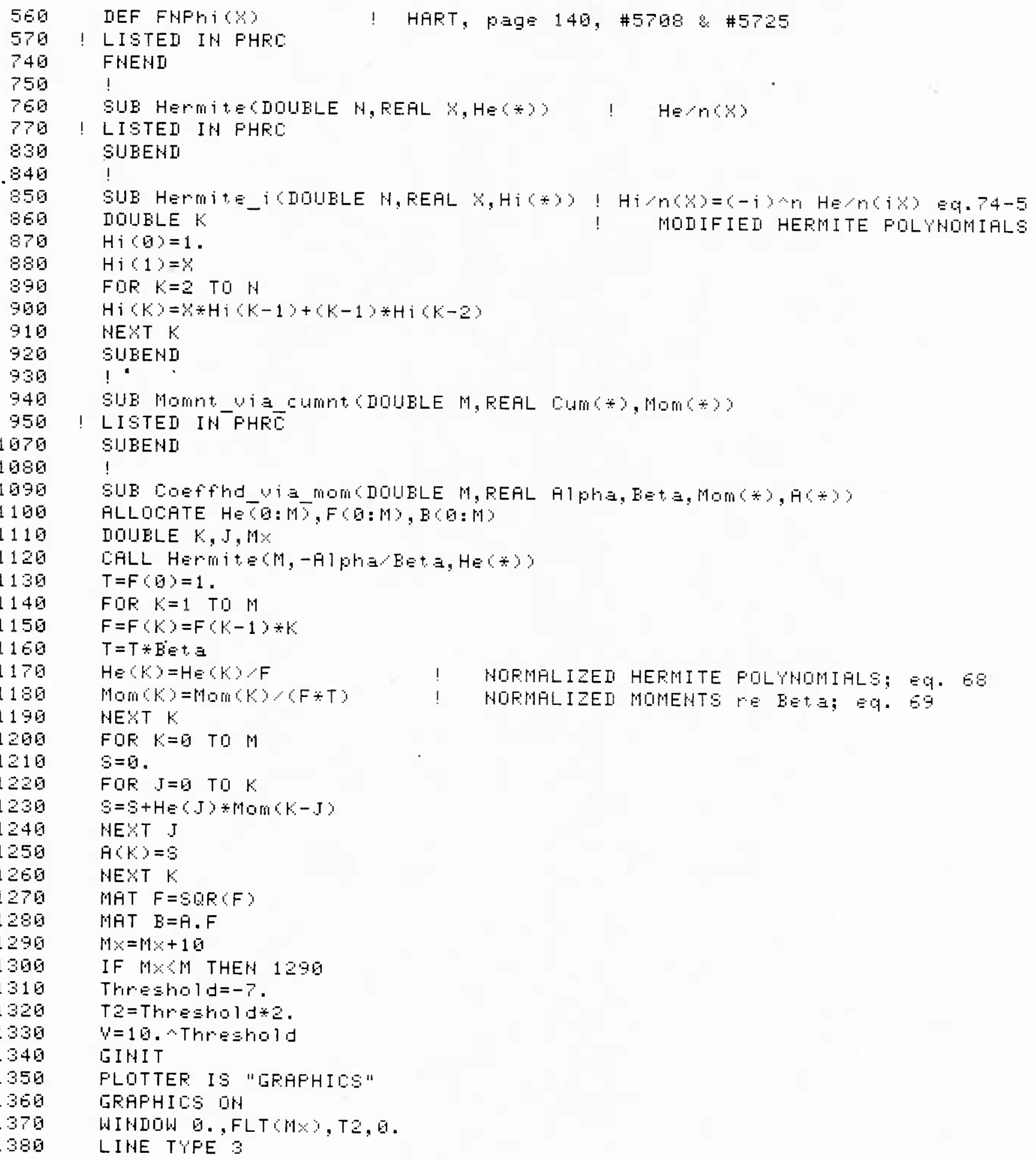




\section{PROGRAM PHDMandRM (cont'd)}

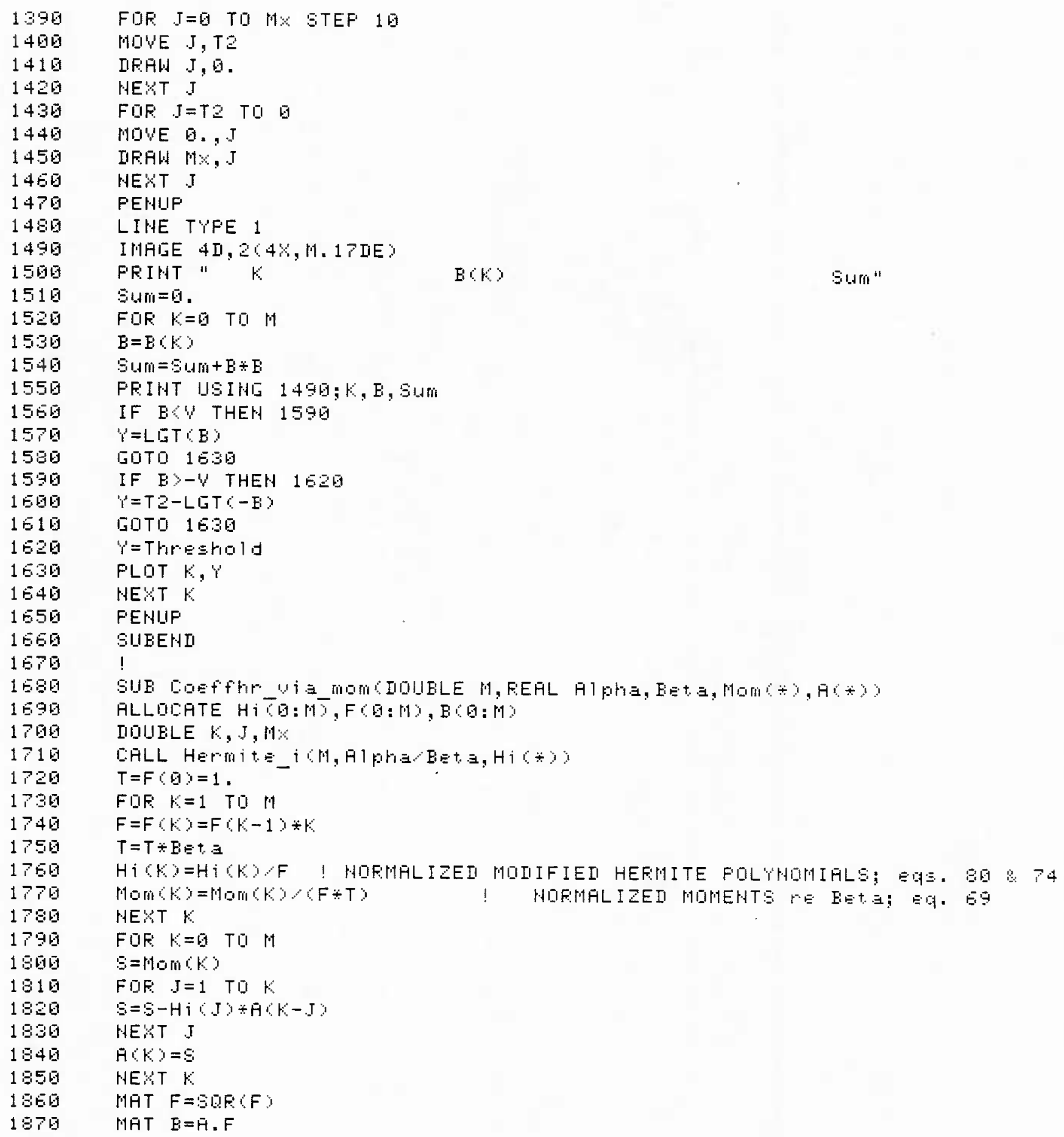




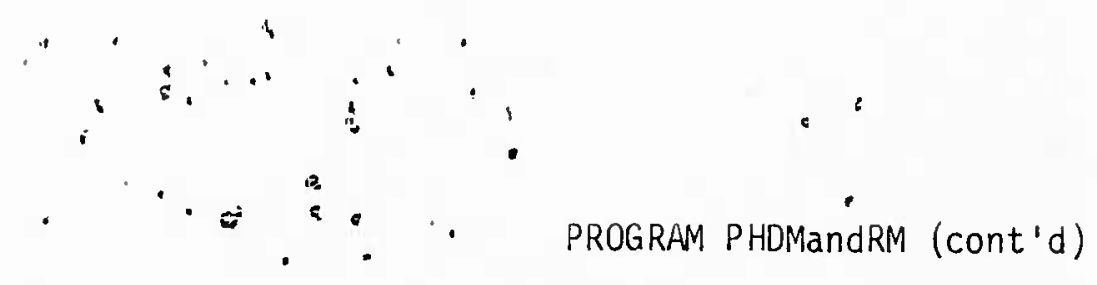

TR 7377

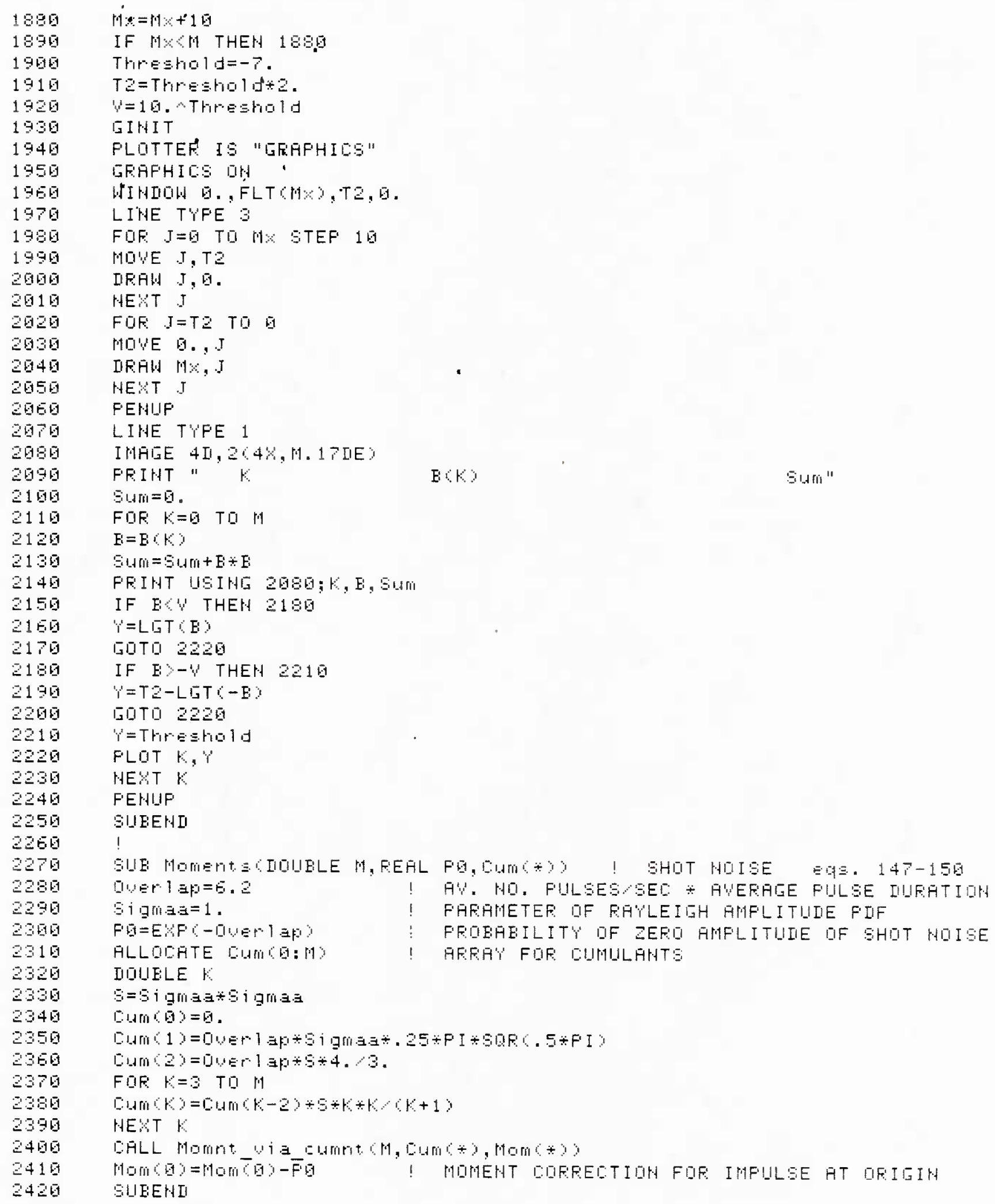


TR 7377

\section{PROGRAM pHDMandRM}

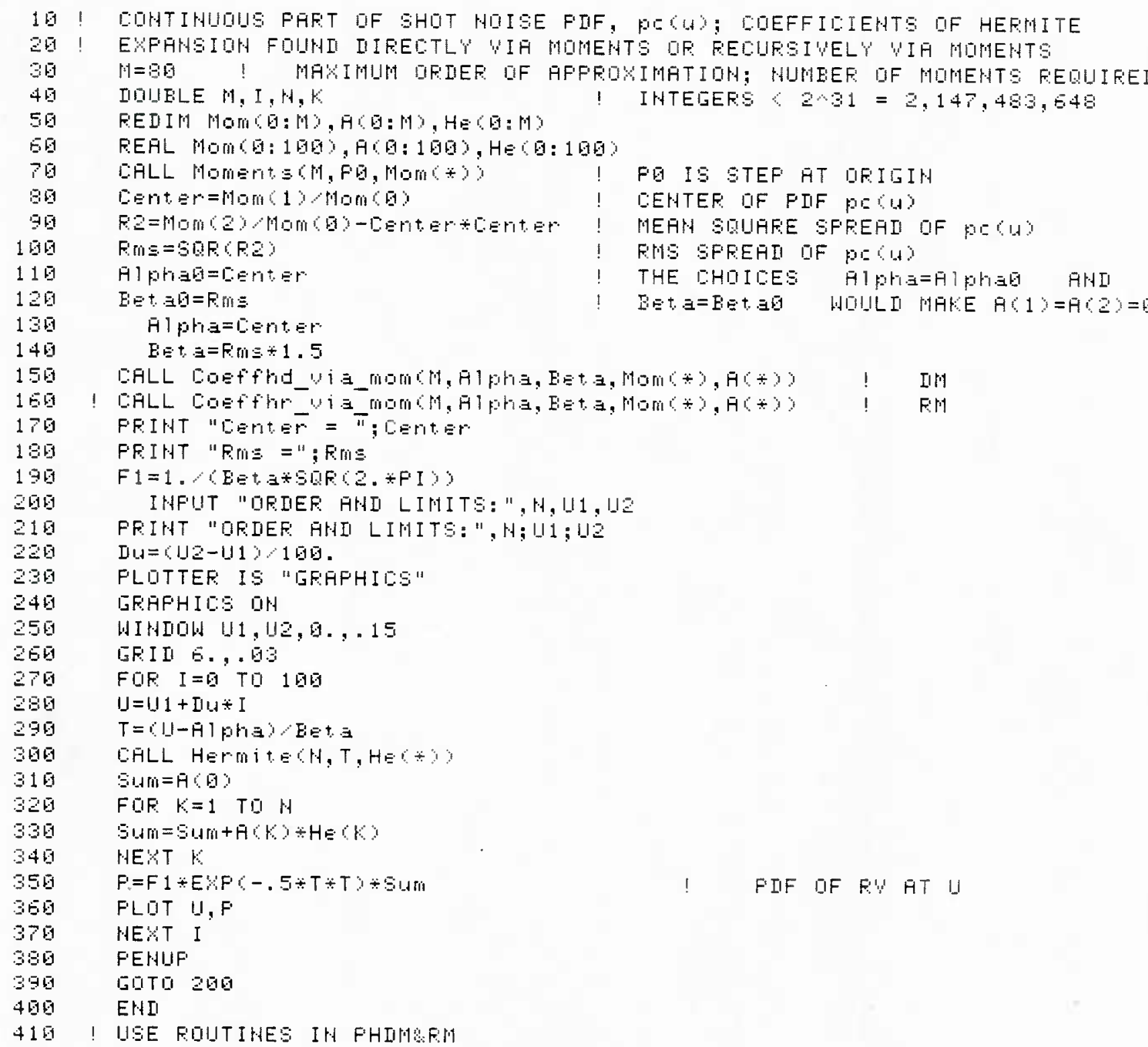




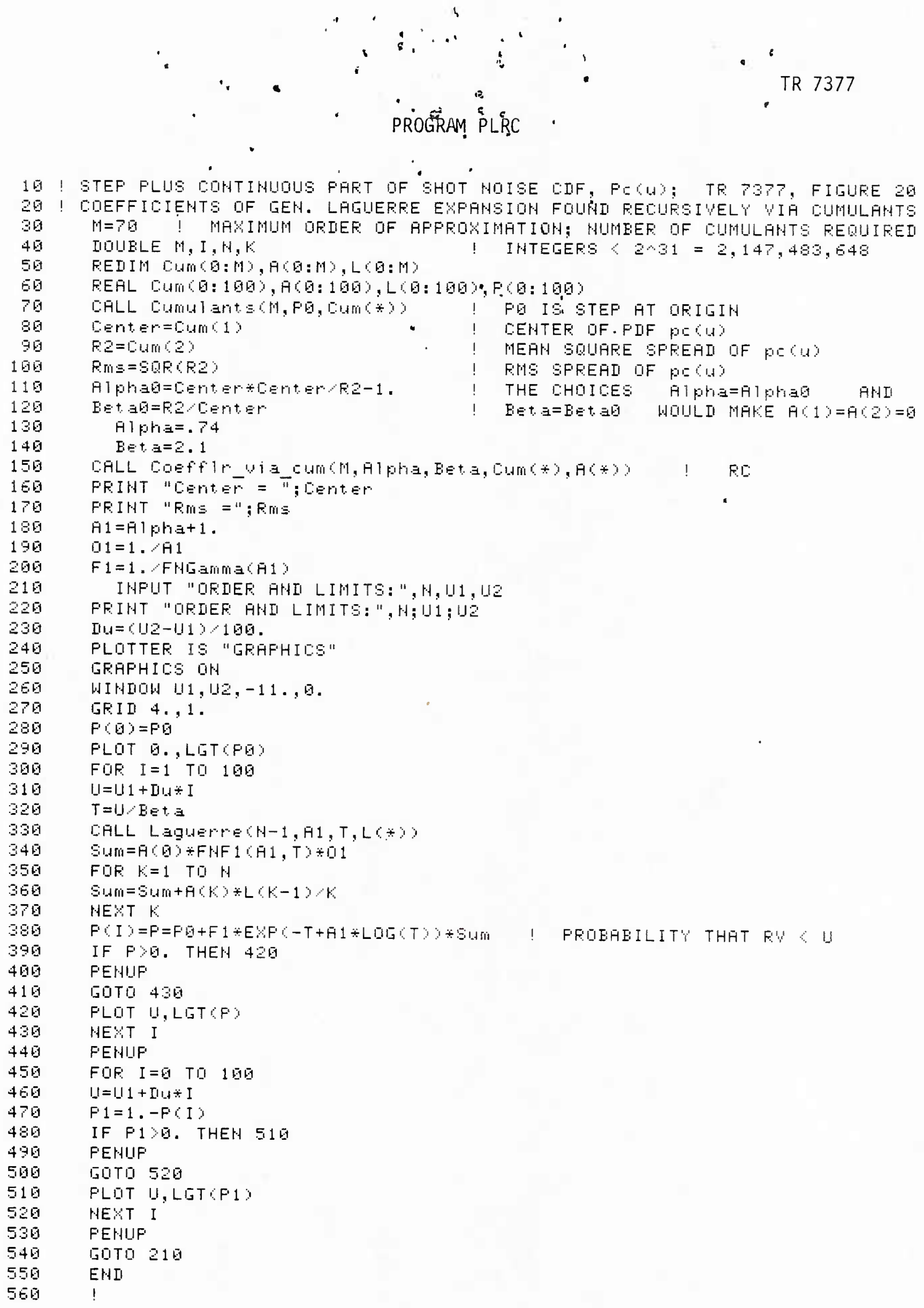


PROGRAM PLRC (cont'd)

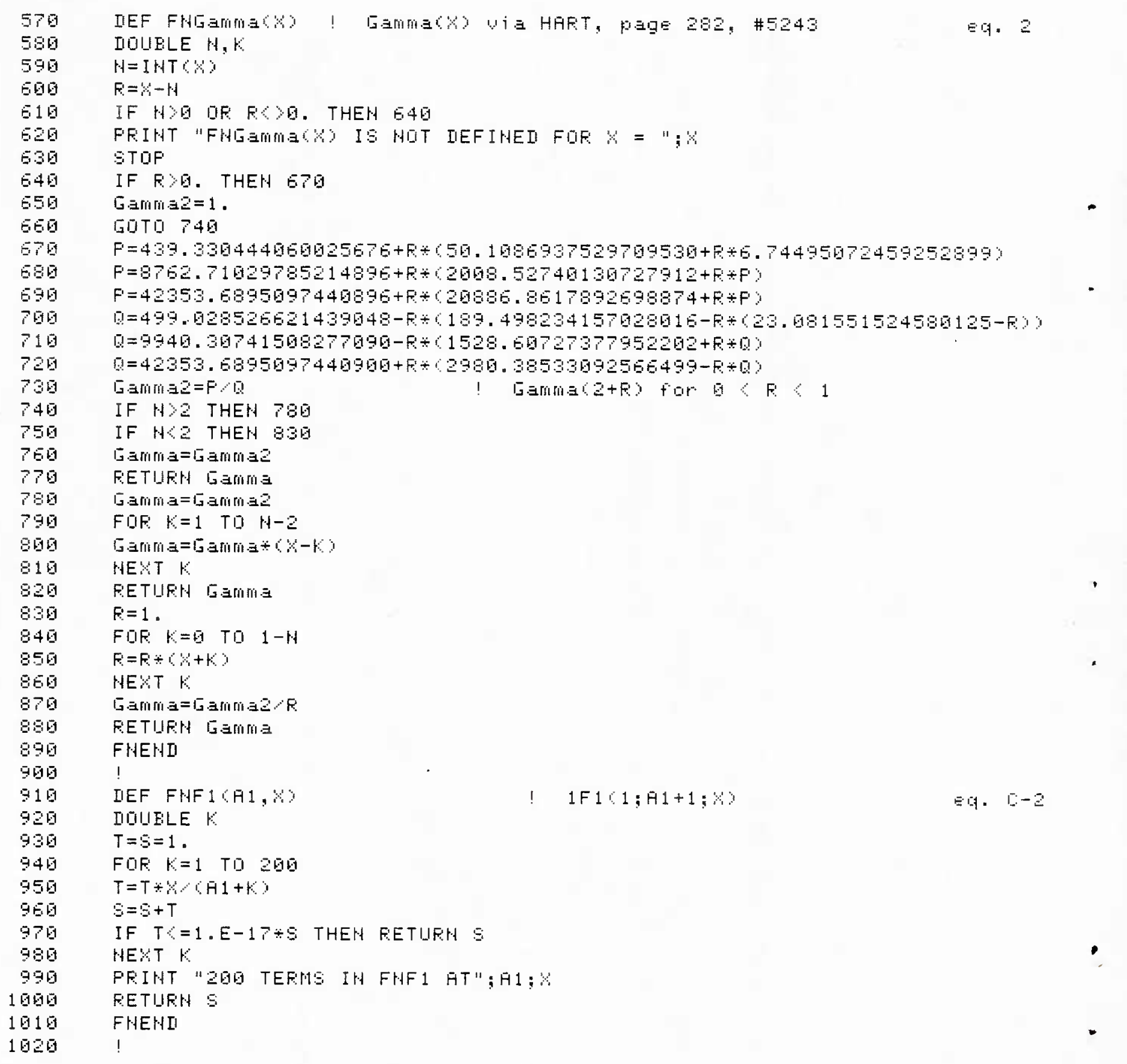




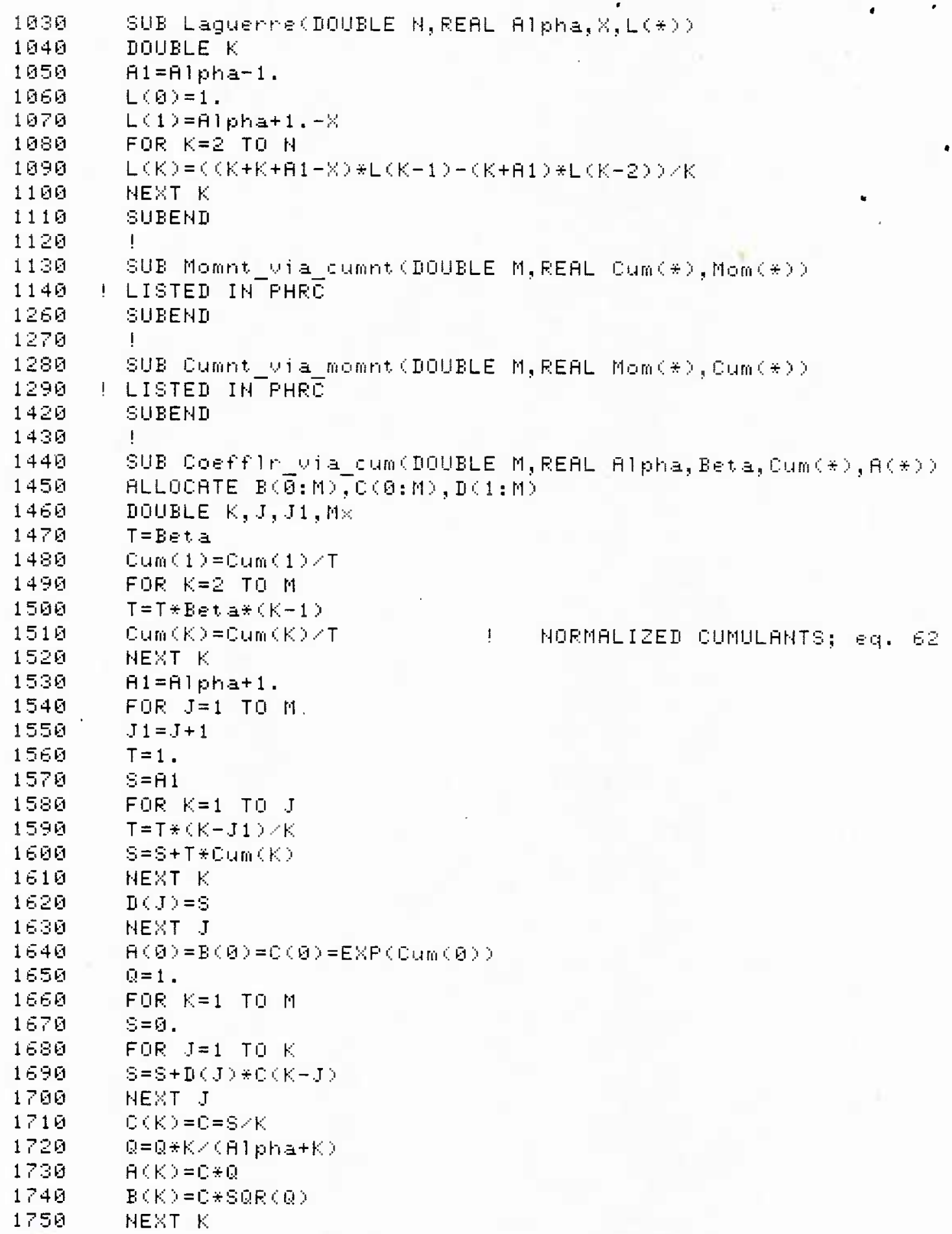

1039

1946

1650

165

1979

1656

1696

1100

1119

1120

$11: 5$

1145

$12 \mathrm{E}$

1275

1280

1296

1420

1430

1446

1456

1450

1479

1435

1496

156

1516

1529

$15: 50$

1540

1556

156

1578

1586

1596

16 更可

1615

160

1 娄目

1540

1650

$1 E B$

1670

1680

1690

1700

1716

170

1736

1746

1758

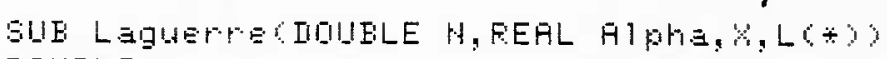

IIIIIELE K

$\vec{H} 1=\bar{A} 1$ pha $\mathrm{B}-1$.

$L(\theta)=1$.

L(1) =A1pha+1. $-Y$

FDR $k=2 \mathrm{TOH}$

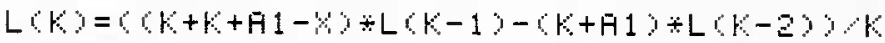

HEYT K

BUEEHII

!

SUE MGMrt

! LISTEII IN FHFE:

SUEEHI

!

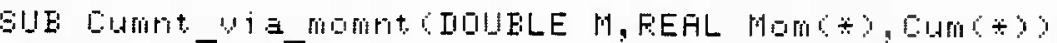

! LISTEI IH FHFE:

SUEEHII

!

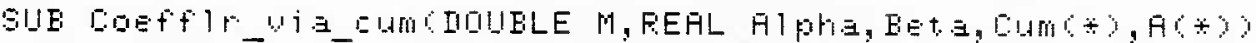

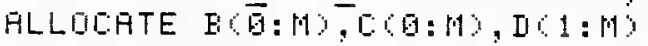

IIDUELE K,J,J1, M\%

$\mathrm{T}=\mathrm{E} E \mathrm{E} \mathrm{t} \cdot \mathrm{a}$

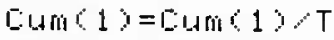

FDF:

$T=T * E=E \div(K-1)$

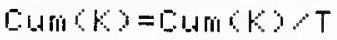

NEXT K

$\hat{\mathrm{A}} 1=\mathrm{H} 1$ phi $\mathrm{a}+1$.

FDE $J=1$ TO

$. T 1=. T+1$

$T=1$.

$S=A 1$

FDE $K=1$ TD J

$T=T \div 6 K-J 1)<$

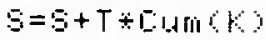

HEXT $K$

II 6.7$)=5$

HEST IT

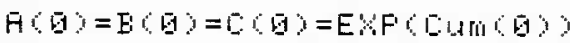

$\bar{l}=1$.

FIF $K=1$ TO

$S=0$.

FIR $J=1$ TO

$S=S+I)(2)+C<-2)$

HEYT .T

$\mathrm{LCO}=\mathrm{L}=5 \mathrm{~K}$

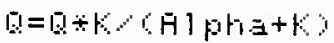

$\mathrm{H} C \mathrm{~B} \mathrm{C}=\mathrm{Z} \div \mathrm{O}$

$\mathrm{E} C \mathrm{~K} \mathrm{O}=\mathrm{E} \div \mathrm{SEC}(\mathrm{D})$

HEST K

1 HOFMALIEI EUMILAHTS: EG. ES 
TR 7377

PROGRAM PLRC (cont'd)

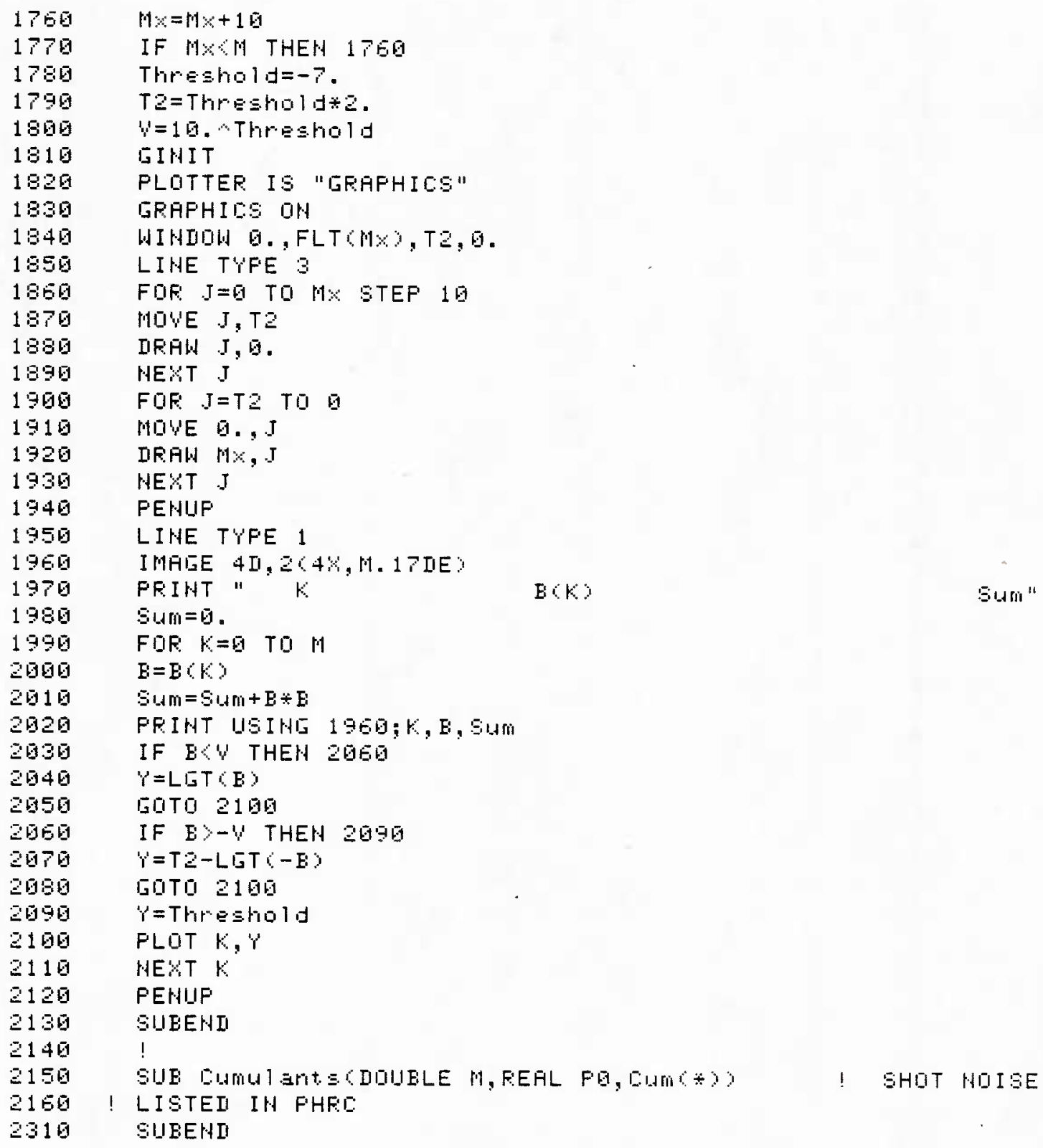


PROGRAM PLRC

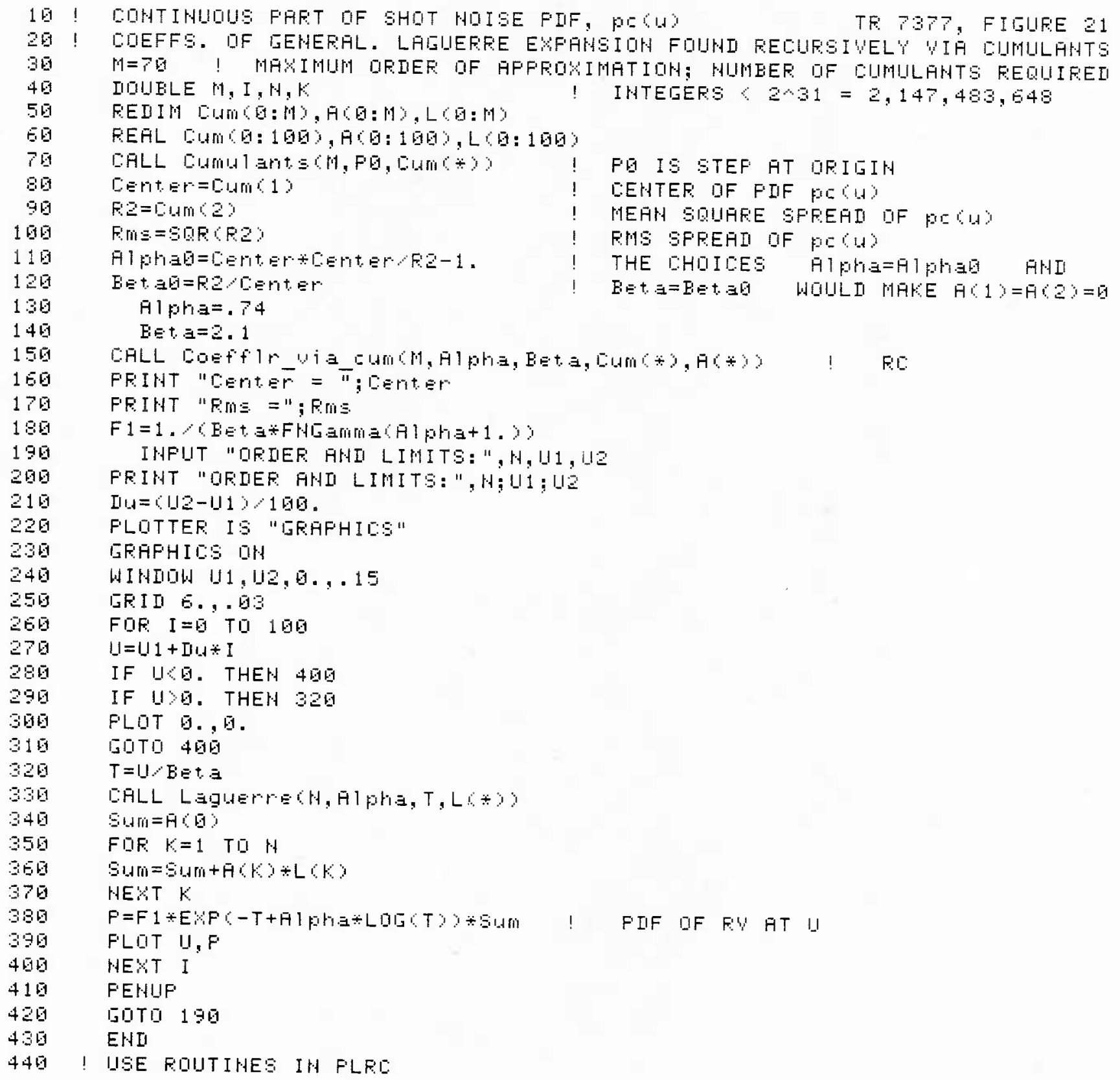




\section{PROGRAM PLDMandRM}

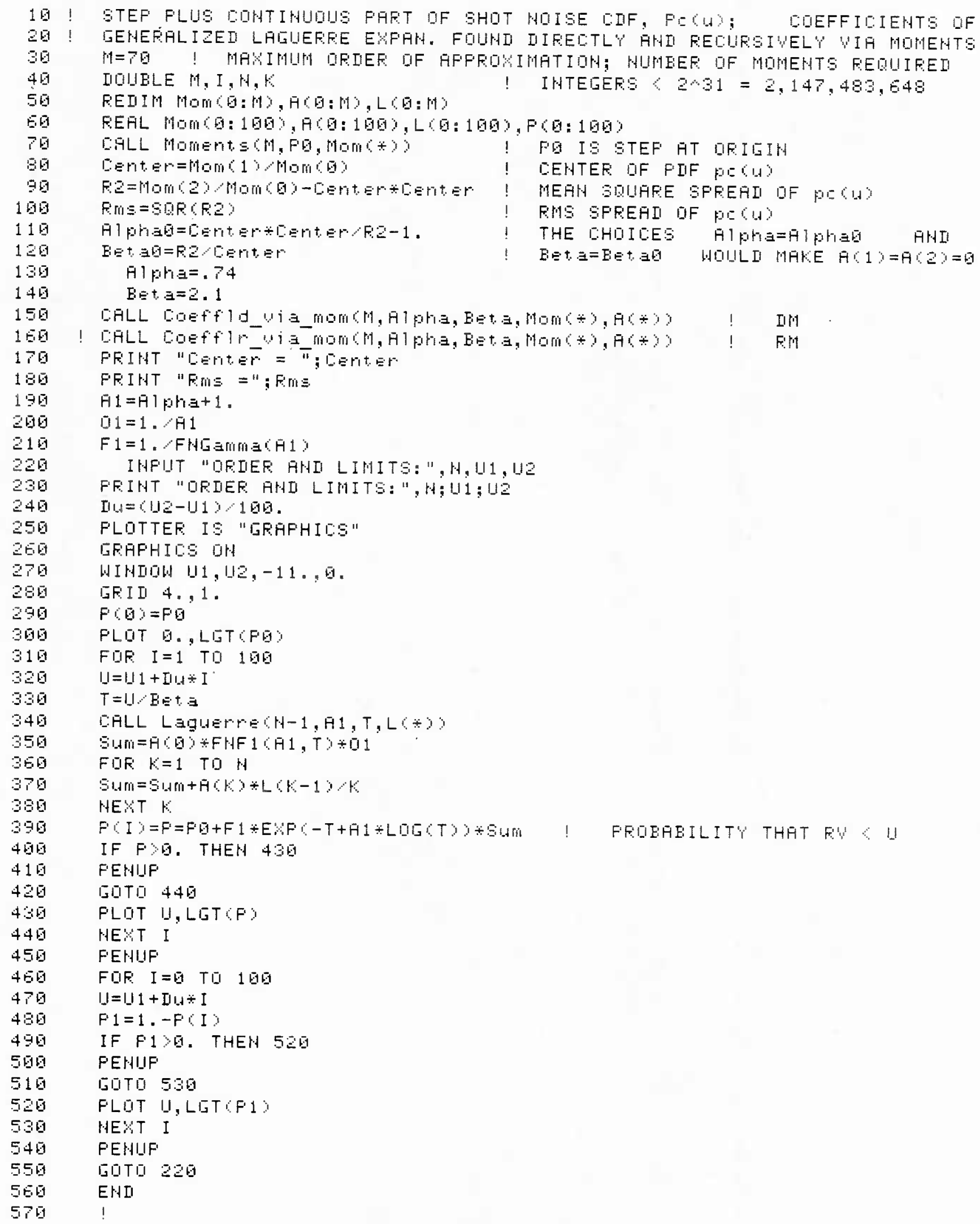


PROGRAM PLDMandRC (cont'd)

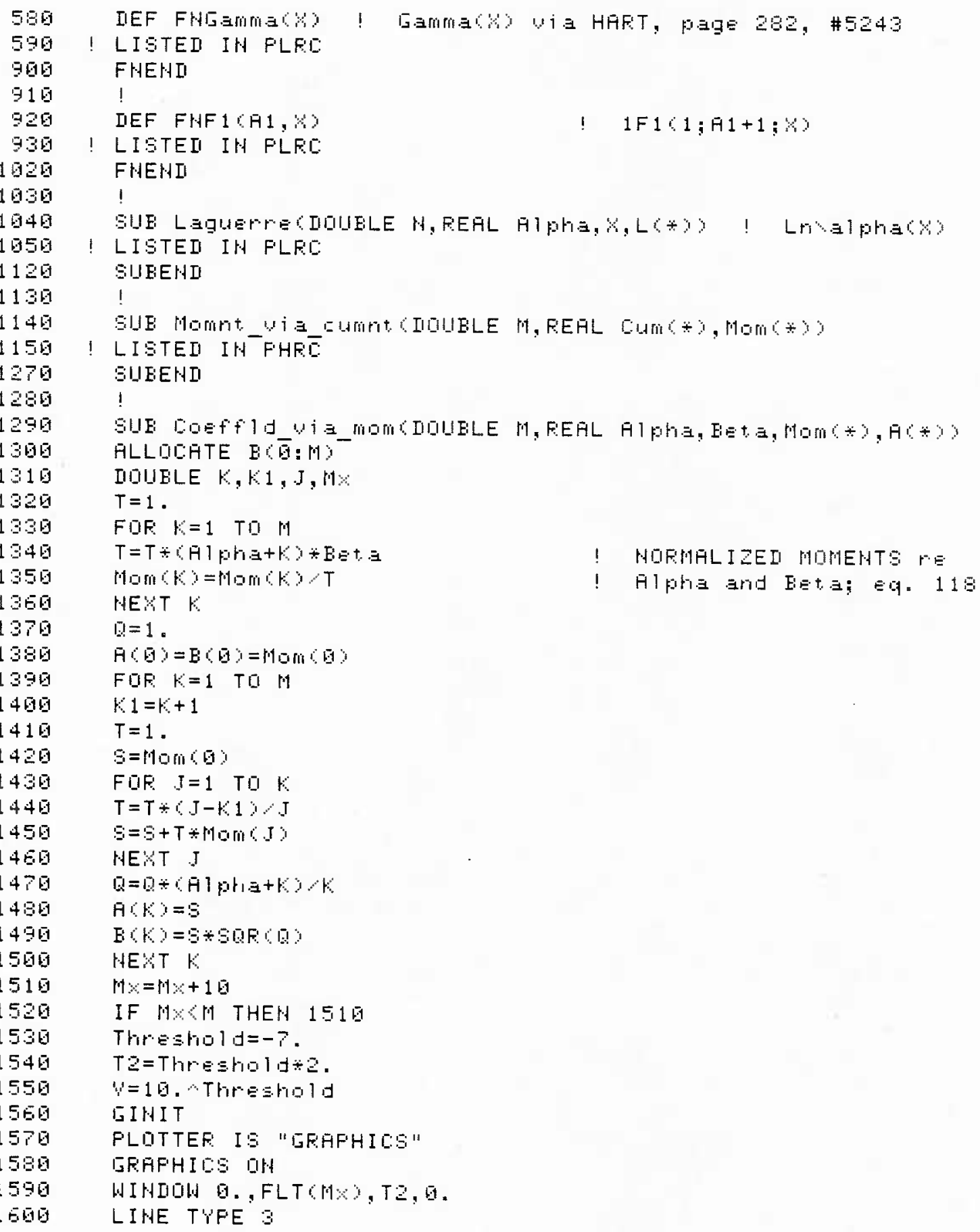


TR 7377

PROGRAM PLDMandRM (cont'd)

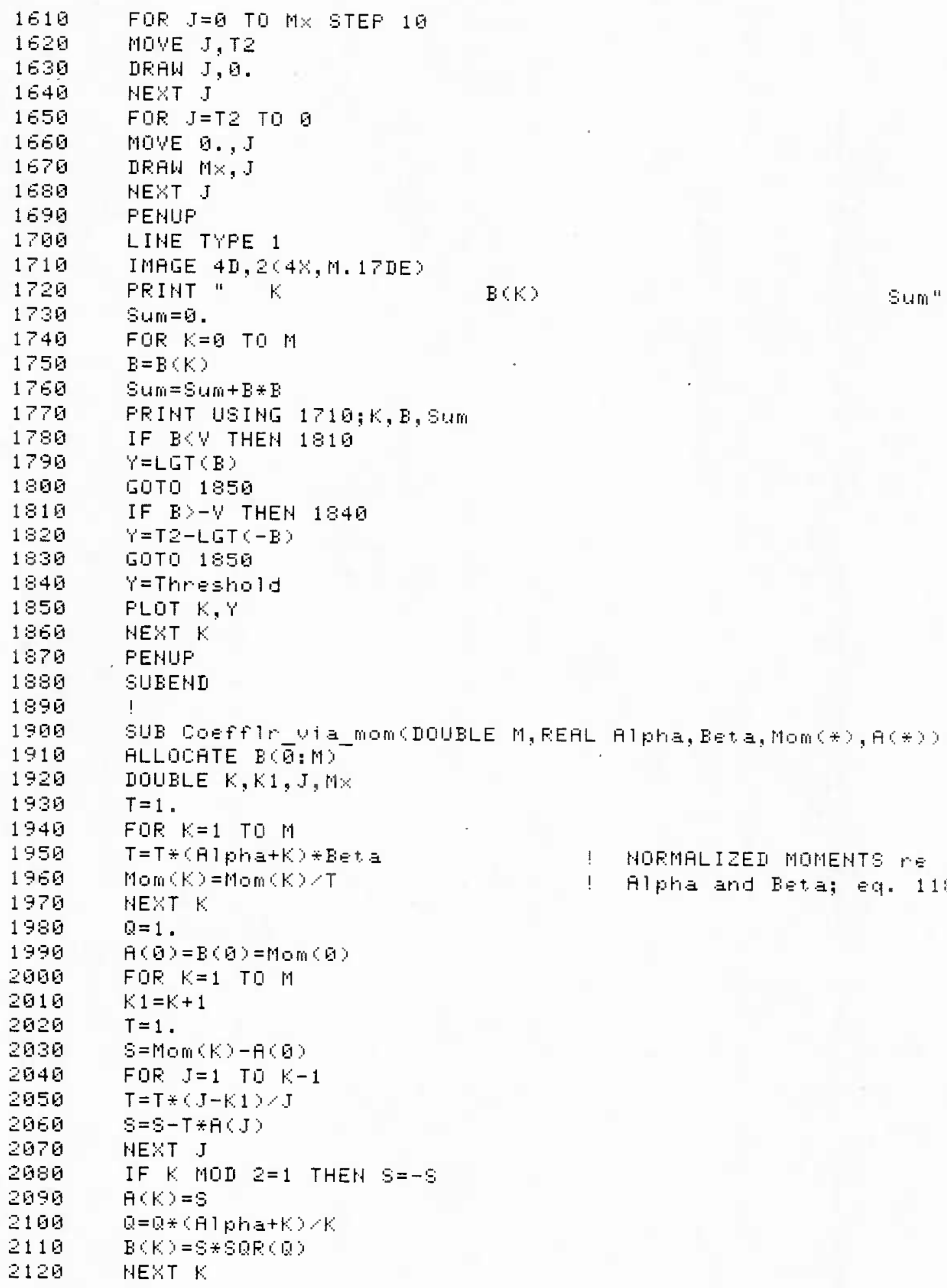


PROGRAM PLDMandRM (cont'd)

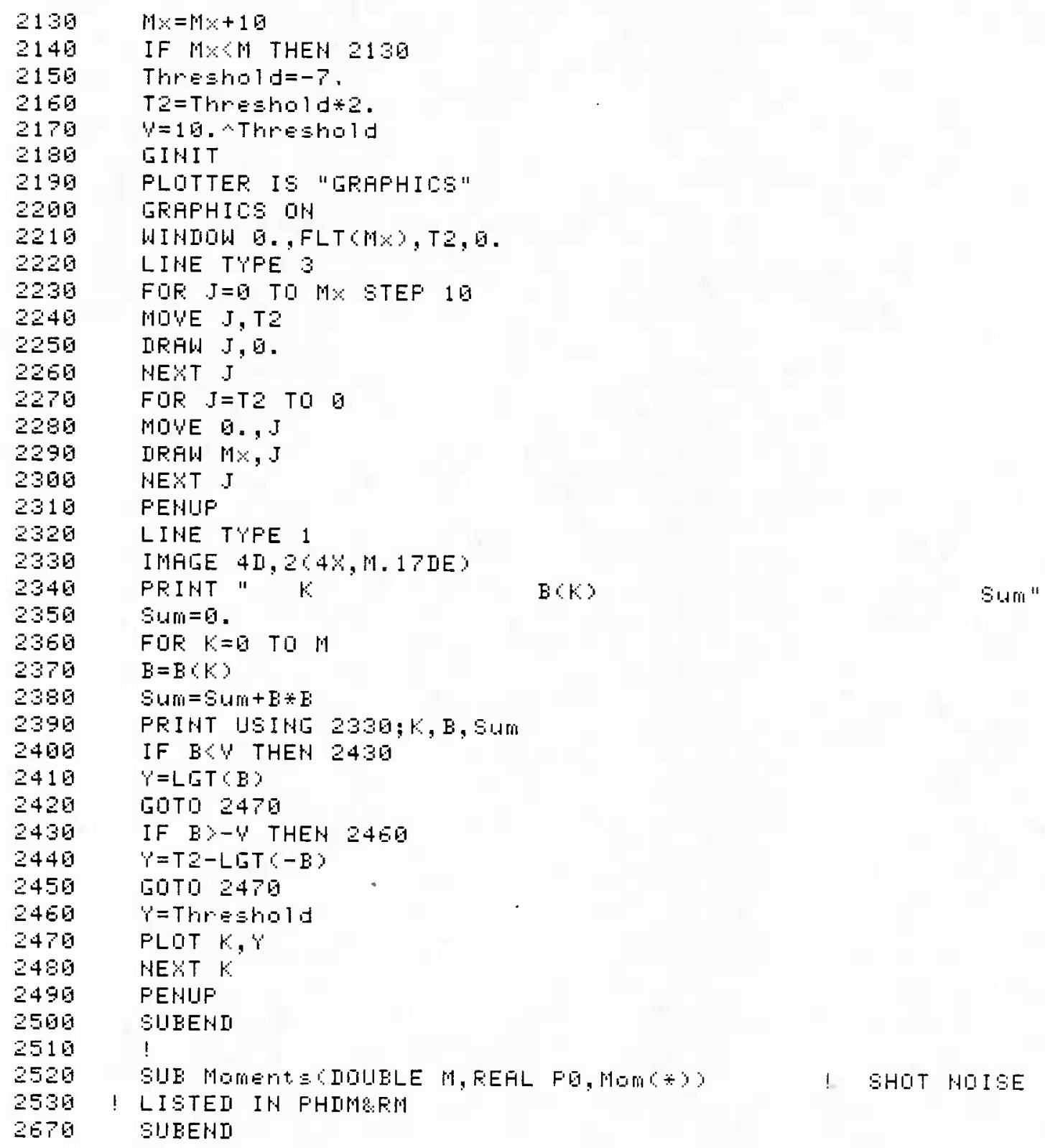




\section{PROGRAM pLDMandRM}

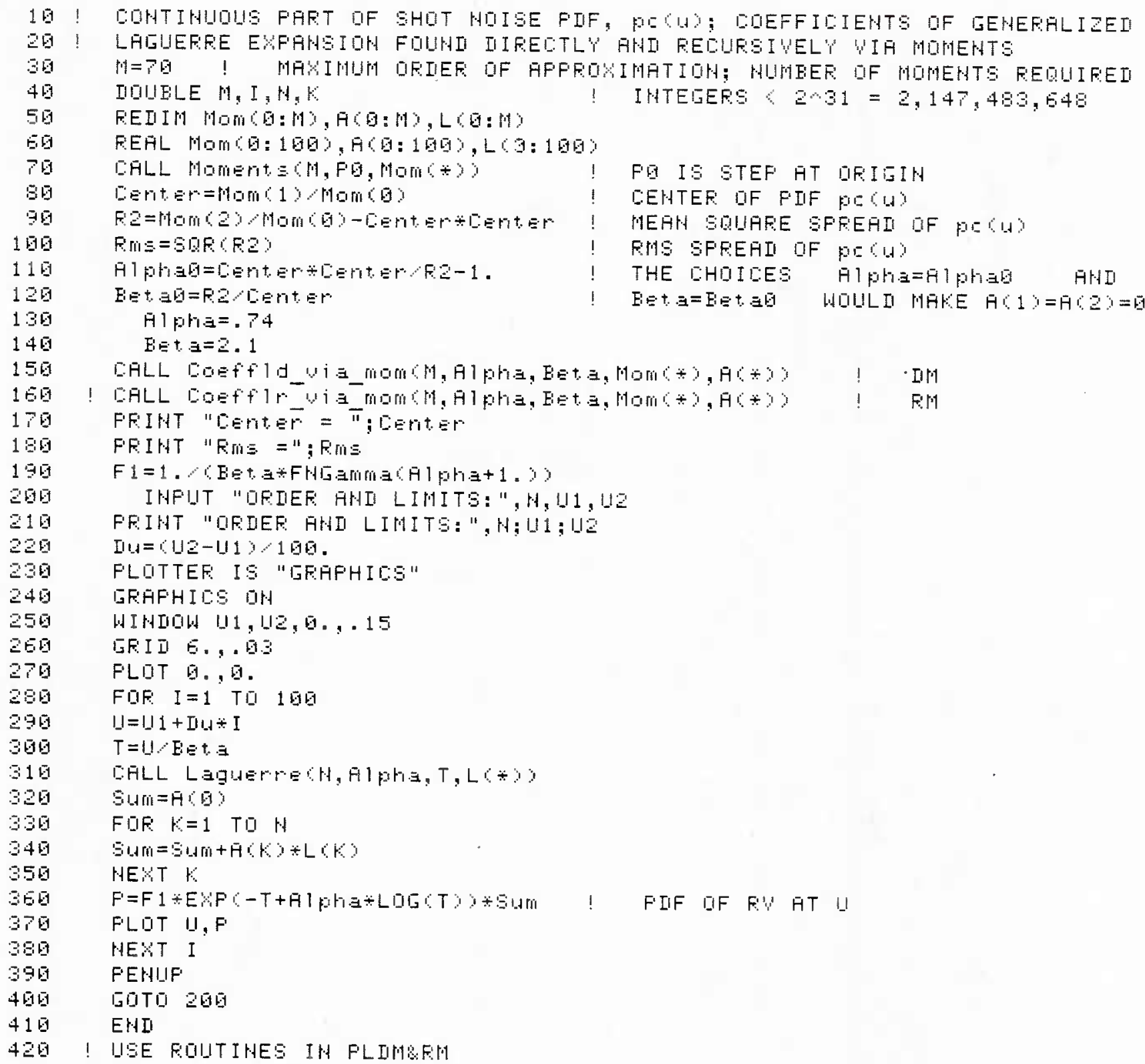




\section{REFERENCES}

1. J. I. Marcum, "A Statistical Theory of Target Detection by Pulsed Radar: Mathematical Appendix, "Research Memorandum RM-753, RAND Corp., Santa Monica, Calif., 1 July 1948. Also in IRE Trans. on Information Theory, Vol. IT-6, No. 2, April 1960.

2. H. Cramér, Mathematical Methods of Statistics, Princeton University Press, Princeton, N. J., 1961.

3. M. G. Kendall and A. Stuart, The Advanced Theory of Statistics; Vol. 1, Distribution Theory, Hafner Publishing Co., N. Y., 3rd edition, 1969.

4. A. H. Nutta11, "Under-Ice Roughness: Shot Noise Model," IUUSC Technical Memorandum 841208, 31 December 1984.

5. Handbook of Mathematical Functions, U. S. Department of Commerce, National Bureau of Standards, Applied Mathematics Series No. 55, U. S. Government Printing Office, June 1964.

6. A. H. Nutta11, "Recursive Inter-Relationships Between Moments, Central Moments, and Cumulants, "NUSC Technical Memorandum No. TC-201-71, 12 October 1971.

7. G. Szegö, Orthogonal Polynomials, American Mathematical Society, Vol. 23, Providence, R. I., 3rd edition, 1967. 
8. I. S. Gradshteyn and I. M. Ryzhik, Table of Integrals, Series, and Products, Academic Press, Inc., New York, 1980.

9. A. H. Nuttall and B. Dedreux, "Exact Operating Characteristics for Linear Sum Of Envelopes of Narrowband Gaussian Process and Sinewave," NUSC Technical Report 7117, 11 January 1984.

10. A. H. Nutta11, "Accurate Efficient Evaluation of Cumulative or Exceedance Probability Distributions Directly from Characteristic Functions," NUSC Technical Report 7023, 1 October 1983.

11. A. H. Nutta11, "Exact Performance of General Second-Order Processors for Gaussian Inputs," NUSC Technical Report 7035, 15 October 1983. 
INITIAL DISTRIBUTION LIST

Addressee

No. of Copies

ASN (RE\&S)

OUSDR\&E (Research and Advanced Technology)

Deputy USDR\&E (Res \& Adv Tech)

OASN, Spec Dep for Adv Concept

Principal Dep Assist Secretary (Research)

OASN, Dep Assist Secretary (Res \& Applied Space Tech)

ONR, ONR-100, $-102,-200,-400,-410,-411$ (N. Gerr), $-422,-425 \mathrm{AC},-430$

CNO, OP-098, $-941,-951$

CNM, MAT-00, $-05,-05$ (G. Morton)

DIA, DT-2C

NRL, Code 5162 (Nate Yen), (A. A. Gerlach)

NRL, USRD

NORDA

USOC, Code 241,240

SUBBASE LANT

NAVSUBSUPACNLON

NAVOCEANO, Code 02 (1)

NAVAIRSYSCOM

NAVELECSYSCOM, ELEX 03, 310, 320

NAVSEASYSCOM, SEA-00, -05R, -06R, -63R, SEA-92R

NAVAIRDEVCEN, Warminster

NAVAIRDEVCEN, Key West

NOSC, Code 713 (Fredric J. Harris), 8302

NOSC, Code 6565 (Library)

NAVWPNSCEN

NCSC, Code 724

NAVCIVENGRLAB

NAVSWC

NAVSURFWPNCEN, Code U31

NISC

CNET, Code 017

CNTT

NAVSUBSCOL

NAVTRAEQUIPCENT, Technica 1 Library

NAVPGSCOL

NAVWARCOL

NETC

APL/UW, SEATTLE

ARL/PENN STATE, STATE COLLEGE (F. W. Symons)

CENTER FOR NAVAL ANALYSES (ACQUISITION UNIT)

DTIC

DARPA

NOAA/ERL

NATIONAL RESEARCH COUNCIL

WEAPON SYSTEM EVALUATION GROUP

WOODS HOLE OCEANOGRAPHIC INSTITUTION (Dr. E. Weinstein)

ENGINEERING SOCIETIES LIB, UNITED ENGRG CTR

NATIONAL INSTITUTE OF HEALTH 
INITIAL DISTRIBUTION LIST (Cont'd)

Addressee

No. of Copies

ARL, UNIV OF TEXAS

MARINE PHYSICAL LAB, SCRIPPS

UNIVERSITY OF CALIFORNIA, SAN DIEGO

NAVSURWEACTR

DELSI

INTERMS INC.

MAR INC.

$B-K$ DYN INC.

BBN, CAMBRIDGE, MA (H. Gish)

EWASCTRI

HYDROINC (D. Clark)

SUMRESCR ( $M$. Henry)

ANALTECH INC. (G. Bourgond)

ANALTECHNS

GENPHYCORP (M. Bauer)

EDOCORP ( $J$. Vincenzo)

OPERRES INC. (Dr. V. P. Simmons)

TRA CORP. (J. Wilkinson)

BB\&N INC., San Diego

NETS (R. Medeiros)

GESY (D. Bates)

ORI CO., INC. (G. Assard)

Or. Norman Bleistein, Denver, Co

PROMETHEUS INC. (Dr. J.S. Byrnes)

BB\&N INC., New London, CT (Dr. P. G. Cable)

ROYAL MILITARY COLLEGE, Dept. of Electrical Engineering,

Kingston, Ontario, Canada (Prof. Y. T. Chan)

UNIVERSITY OF FLORIOA, Dept. of Electrical Engineering

(D. C. Childers)

SANDIA NATIONAL LAB, Albuquerque, NM (J. Claasen)

COGENT SYSTEMS, INC., Waban, MA (J. P. Costas)

BB\&N INC., Arlington, VA (Dr. H. CoX)

AUTONETICS MARINE SYSTEMS DIV., Anaheim, CA (L. T. Einstein)

ROCKWELL INTERNATIONAL, Anaheim, CA (Dr. O. F. Elliot)

Bernard Harris, Dobbs Ferry, NY

EG\&G, Manassas, VA (Dr. J. Hughen)

MAGNAVOX GOV. \& IND. ELEC. C0., Dept. 525, Fort Wayne, IN

(R. Kenefic)

DREXEL UNIVERSITY, DEPT. OF ELEC. \& COMP. ENG.,

Philadelphia, PA (Prof. S. Kesler)

DEFENCE RESEARCH ESTABL. ATL., Dartmouth, Nova Scotia,

Canada, Library (B. E. Mackey)

SCHLUMBERGER WELL SERVICES ENGINEERING, Houston, TX

(S. L. Marple)

PSI MARINE SCIENCES, New London, CT (Or. R. Mellen)

Dr. David Middleton, New York, NY

UNIVERSITY OF CONNECTICUT, Dept. of Elec. \& Comp. Science

(Prof. C. L. Nikias) 
INITIAL DISTRIBUTION LIST (Cont'd)

Addressee

BB\&N INC., Canoga Park, CA (Dr. A. G. Piersol)

LINK-A-BIT CORP., Lexington, MA (Dr. R. Price)

DALHOUSIE UNIVERSITY, Dept. of Oceanography, Halifax, Nova Scotia, Canada (Dr. B. Ruddick)

UNIVERSITY OF RHODE ISLAND, Dept. of Electrical Engineering (Prof. L. Scharf, Prof. D. Tufts)

YALE UNIVERSITY, Depth of Electrical Engineering (Prof. P. M. Schultheiss)

DEFENSE SYSTEMS, INC., MCLean, VA (Or. G. S. Sebestyen)

NATO SACLANT ASW RESEARCH CENTRE (Dr. E. J. Sullivan, Library)

DEFENCE RESEARCH EST. PACIFIC, Canada (Dr. D. J. Thomson)

Robert J. Urick, Silver Spring, MD

RCA/GOVERNMENT SYSTEMS DIV., Moorestown, NJ (H. Urkowitz)

TEL-AVIV UNIVERSITY, Dept. of Electronic Systems, Te1-Aviv, Israel (Prof. E. Weinstein)

TRACOR, Inc., Analysis \& App 1. Res. Div., Austin, TX

(J. F. Wilkinson)

COAST GUARD ACADEMY, Dept. of Mathematics (Dr. J. J. Wolcin) UNIVERSITY OF IOWA, Elec. \& Comp. Eng. Dept.
No. of Copies

1 
U219360

1 